WHC-EP- -0299

DE91 001289

\title{
Animal Intrusion Status Report for Fiscal Year 1989
}

D. S. Landeen

Date Published

August 1990

Prepared for the U.S. Department of Energy Office of Environmental Restoration and Waste Marnagement

\section{(W) Westinghouse \\ P.O. Box 1970 \\ Hanford Company Richland, Washington 99352}

Hanford Operations and Engineering Contractor for the

U.S. Department of Energy under Contract DE-AC06-87RL10930 
WHC-EP-0299

\section{ANIMAL INTRUSION STATUS REPORT FOR FISCAL YEAR 1989}

\section{ABSTRACT}

The Protective Barrier and Warning Marker System Development Plan* identified tasks that need to be completed to design a final protective barrier to implement in-place disposal of radioactive waste. This report summarizes the animal intrusion tasks that were conducted by Westinghouse Hanford Company in fiscal years 1988 and 1989 with respect to small mammals and water infiltration.

An animal intrusion $7 y$ simeter facility was constructed and installed in fiscal year 1988. The facility consists of two outer boxes buried at grade that serve as receptacles for six animal intrusion 7ysimeters. Small burrowing mammals common to the Hanford Site environs are introduced over a 3- to 4-month period. Supplemental precipitation is added to three of the lysimeters with a rainulator at a rate equivalent to a 100-year storm. Soil moisture samples are taken before and after each test and soil moisture measurements are also taken with a hydroprobe during the test period.

Two tests have been completed and a third test is in progress. Preliminary results from the first test indicate that the additional water, that was supplemented by the rainulator to the plots with burrowing animals, is being removed. Mechanisms that might account for this include evaporation loss caused by continuous soil turnover from burrow excavations and moisture loss from open burrows, which probably allow some air circulation.

Data from the second test, which was conducted during the winter, indicate that all the plots (control and animal) gained water. Plots with animal burrows gained almost twice as much water as the control plots. The greatest changes occurred at the 18-in. level in all the plots. Additional tests, scheduled to be conducted over the next few years, should substantiate or verify these preliminary observations.

*Adams, M. R. and N. R. Wing, 1986, Protective Barrier and Warning Marker System Development Plan, RHO-RE-PL-35P, Rockwell Hanford Operations, Richland, Washington. 


\section{EXECUTIVE SUMMARY}

The Protective Barrier and Warning Marker System Development Plan* identified tasks that need to be completed to design a final protective barrier to implement in-place disposal of radioactive waste. Some of the tasks focused on the need to evaluate the impacts that burrowing animals could have on the integrity of a protective barrier system. This report summarizes the animal intrusion tasks that were conducted by Westinghouse Hanford Company in fiscal years (FY) 1988 and 1989 with respect to small mammals and water infiltration.

An animal intrusion $7 y$ simeter facility was constructed and installed near the Field Lysimeter Test Facility (FLTF) adjacent to the Hanford Meteorological station in FY 1988. The FLTF consists of two outer boxes buried at grade that serve as receptacles for six animal intrusion lysimeters. At the beginning of each test the lysimeters are lined with a plastic liner and filled with a fine soil from the McGee Ranch, a soil site near the intersections of State Highway 24 and 240. Small burrowing mammals common to the Hanford Site environs are introduced over a 3-to 4-mo period and allowed to burrow. During the course of each test supplemental precipitation is added to three of the lysimeters with a rainulator, at a rate equivalent to a 100-yr storm. Soil moisture samples are taken before and after each test, while soil moisture measurements are taken with a hydroprobe during the test period.

${ }^{\star}$ Adams, M. R. and N. R. Wing, 1986, Protective Barrier and Warning Marker System Development Plan, RHO-RE-PL-35P, Rockwell Hanford Operations, Richland, Washington. 
At the present time, two tests have been completed and a third test is in progress. Preliminary results from the first test indicate that the additional water, which was supplemented by the rainulator to the plots with burrowing animals, is being removed. The data do not indicate that water movement at depth is significantly greater in plots with burrows over a 3- to 4-mo period. The water does appear to go deeper initially in plots with open burrows, but is soon removed. Mechanisms that might account for this include evaporation loss caused by continuous soil turnover from burrow excavations and moisture loss from open burrows, which probably allow some air circulation.

Data from the second test, which was conducted during the winter, show that all plots (control and animal) gained water. Plots with animal burrows gained almost twice as much water as the control plots. The greatest changes occurred at the 18-in. level in all the plots. Additional tests that are scheduled to be conducted over the next few years should verify or substantiate these preliminary observations. 
WHC-EP-0299

\section{CONTENTS}

1.0 INTRODUCTION . . . . . . . . . . . . . . . . . . . . . 1

2.0 WATER INFILTRATION IN RESPONSE TO SMALL MAMMAL BURROWING . . . . 1

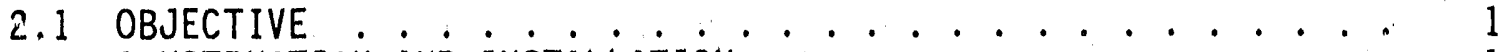

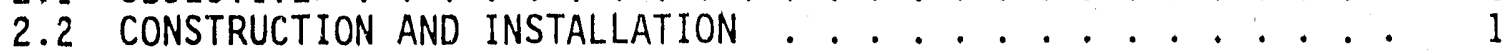

3.0 TEST 1 . . . . . . . . . . . . . . . . . . . . 4 3.1 RESULTS . . . . . . . . . . . . . . . . 11 3.2 SUMMARY OF TEST $1 \ldots \ldots \ldots 11$

4.0 TEST $2 \ldots \ldots \ldots 1 . \ldots \ldots$

4.1 RESULTS . . . . . . . . . . . . . . . . 20

4.2 SUMMARY OF TEST $2 \ldots \ldots 20$

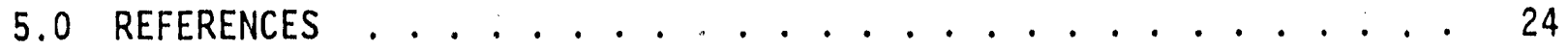
APPENDIXES:

A. Soil Resistance Data ............. A-i

B. Test 1 Graphs ................ B-i

C. Test 2 Hydroprobe Data .............. C

D. Test 2 Soil Moisture Graphs ............ D-i 


\section{LIST OF FIGURES}

1 Animal Intrusion Facility Location ............. 2

2 Barrier Design Flow Diagram . . . . . . . . . . . . 3

3 Location of the McGee Ranch Soil Site . . . . . . . . . . . 5

4 First Animal Intrusion Lysimeter Configuration . . . . . . 8

5 Soil Moisture Profile with Rainulator . . . . . . . . . . . . 12

6 Second Animal Intrusion Lysimeter Configuration . . . . . . . . 13

7 Soil Moisture Profile of the Pocket Mouse . . . . . . . . . . 23

8 Soil Moisture Profile of the Pocket Gopher . . . . . . . . . 23

\section{LIST OF TABLES}

1 Soil Moisture Samples Collected April 13 and 14, 1988, at the Installation .................. 6

2 Soil Moisture Samples Collected October 1988 at the Completion of Test 1........................ 9

3 Amount of Excavated Soil collected at the End of Tests 1 and 2 in the Animal Intrusion Lysimeters ........ 11

4 Soil Moisture Samples Collected November 18, 1988, at the Installation of the Hydroprobe in Test 2 .......... 14

5 Soil Moisture Samples Collected in Apri1 1989 at the End of Test 2........................ 18

6 Soil Moisture Profite Changes During Test 2 . . . . . . . . 2.1 
WHC-EP-0299

ANIMAL INTRUSION STATUS REPORT FOR FISCAL YEAR 1989

\subsection{INTRODUCTION}

This report summarizes the work that has been conducted relative to small mammals and water infiltration at the animal intrusion lysimeter facility near the Hanford Meteorological Station (Figure 1). This task was identified in the Protective Barrier and Warning Marker System Development Plan (Adams and Wing 1986) as one of many tasks needed to design a final barrier to implement in-place disposal. The smal1-mammal work reported here was conducted by the Westinghouse Hanford Company and is one part of the overall animal intrusion work that will be conducted during the next few years (Figure 2).

At the present time, two tests have been conducted and a third is in progress. This report will summarize the work relative to the first two tests. This study is still in the data-collection phase and as a result only a limited portion of the data have been analyzed. The appendices contained in this report reflect the majority of the data that have been collected to date.

\subsection{WATER INFILTRATION IN RESPONSE TO SMALL MAMMAL BURROWING}

\subsection{OBJECTIVE}

The objective of the task is to determine the degree that small-mammal burrow systems affect the downward movement of water.

\subsection{CONSTRUCTION AND INSTALLATION}

Six animal lysimeters ( $5 \mathrm{ft} \times 5 \mathrm{ft} \times 6 \mathrm{ft}$ deep) and two outer boxes big enough to hold three lysimeters each were constructed by the 200 West Area fabrication shops and carpenter shops in 1987. The six individual animal lysimeters are designed so that they can be disassembled and reused at the end of each test period. The outer boxes were installed in the ground at grade in December 1987, and the six animal intrusion lysimeters were installed in March 1988. The two outer boxes were buried approximately $100 \mathrm{ft}$ apart. 


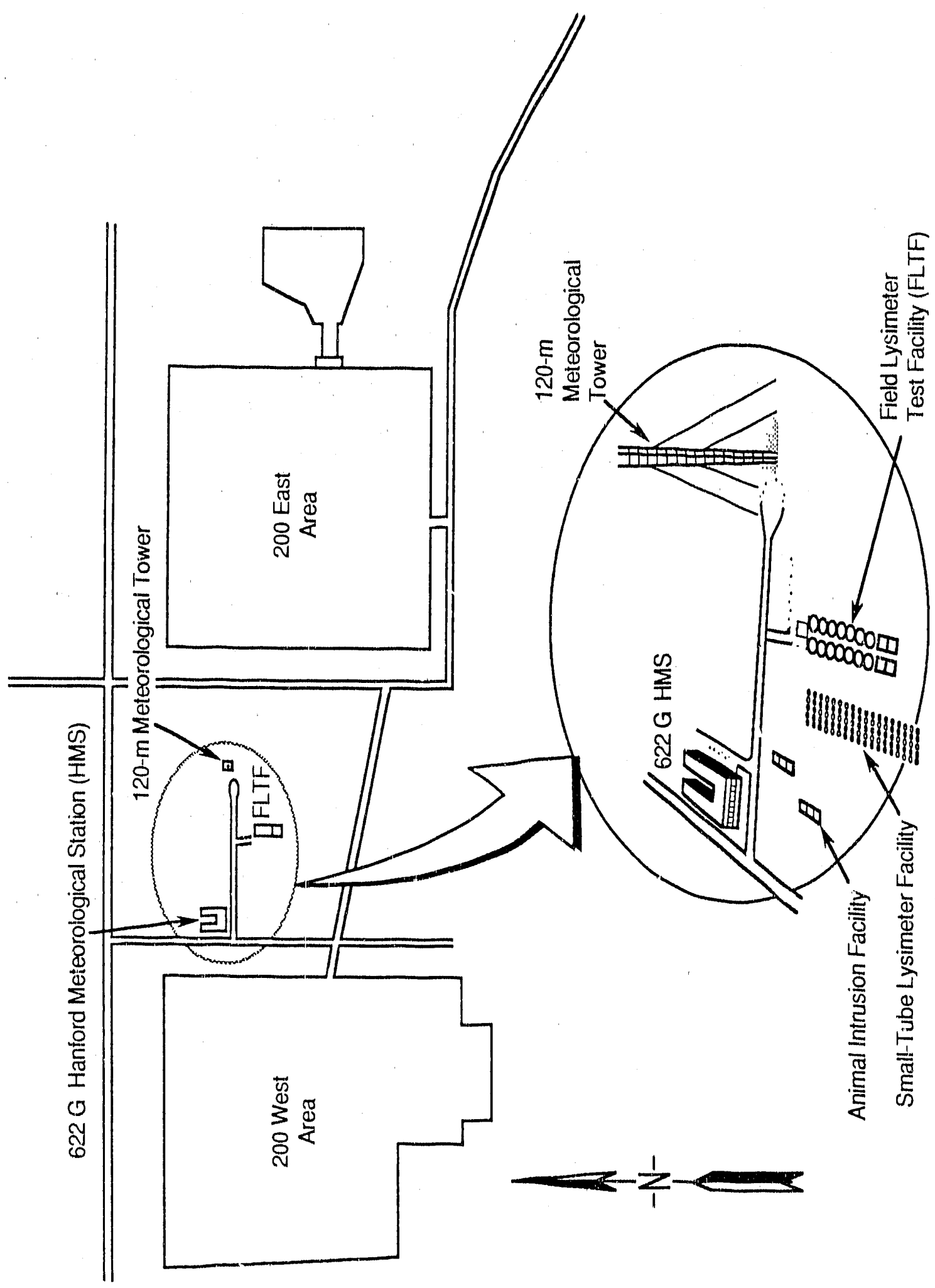

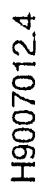




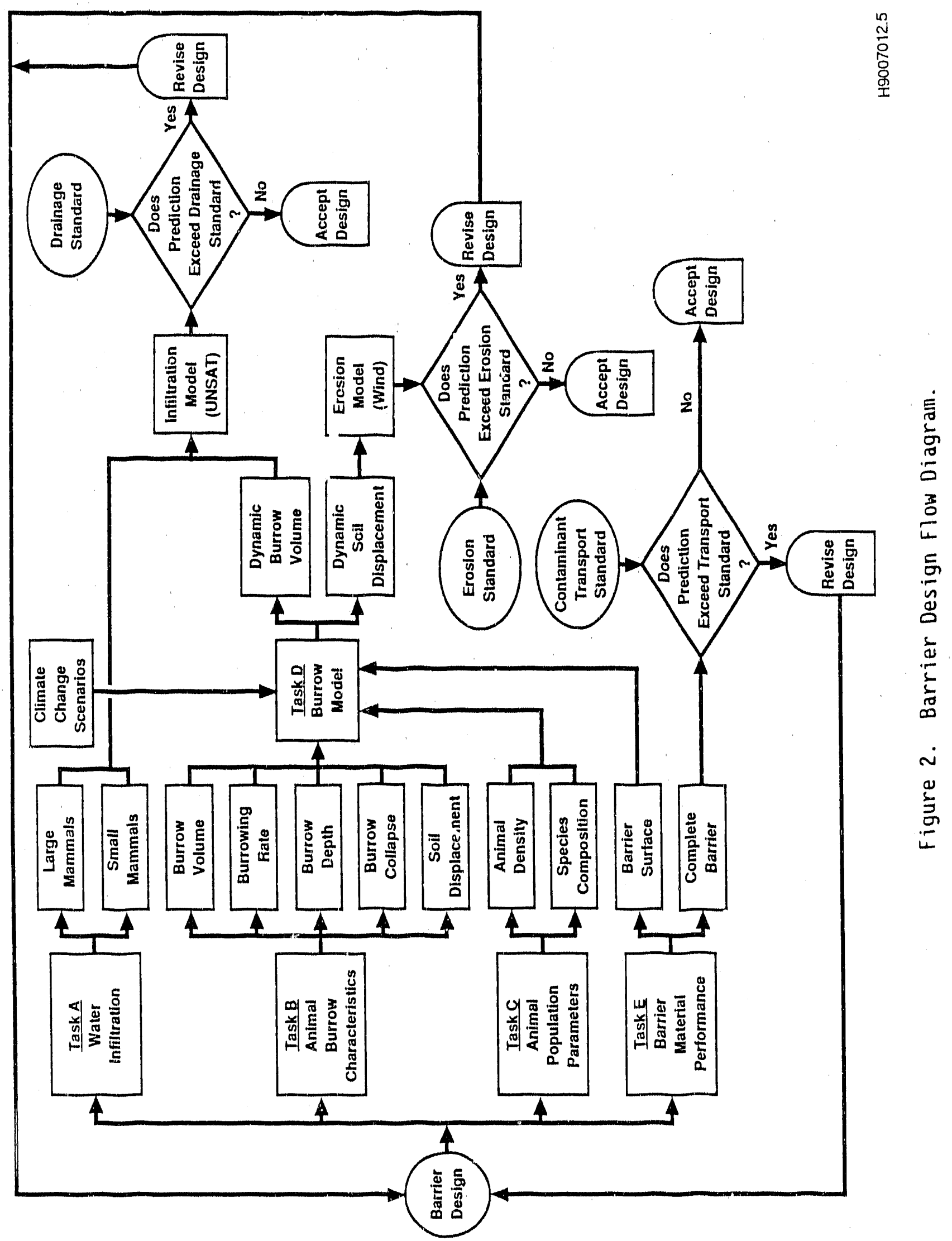


WHC-EP-0299

\subsection{TEST 1}

The following discussion is a summary of the first test that was conducted and preliminary results.

Each lysimeter was lined with a $28-\mathrm{m} 11$ plastic 1 iner and then fllled with 6 to 8 in. of pea gravel. After the boxes were weighed a geotextile material was placed over the gravel and $4 \mathrm{ft}$ of McGee Ranch soil was added in 6-in. lifts. The McGee Ranch is a soil site near the intersection of State Highways 24 and 240 (Figure 3). The fine soil from the McGee Ranch is the soil that has been chosen to construct protective barriers (Last et al. 1987).

The soll was wetted down with approximately $10 \%$ to $12 \%$ water by weight so that each lift could be compacted. A soil moisture sample was collected frolii every $1 \mathrm{ft}$. These soll samples were oven dried to calculate percent moisture by weight (Table 1).

Pacific Northwest Laboratory (PNL) developed soil conductance probes that measure soll resistance. The rationale for using these probes was to obtain in situ nondestructive measurements of gross trends in water movement at depth in each box. The probes measured soil resistance, which is a reflection of soil moisture. The probes were not sensitive enough to measure actual soll moisture, but were only intended to show wetter or dryer conditions at depth. One probe per box was installed during April.

For the first test, two Great Basin pocket mice (Perognathus parvus) and two Townsend ground squirrels (Spermophilus townsendii) were trapped live and released into the lysimeters in June 1988. Figure 4 indicates what boxes the animals were placed into. Boxes 1A, 1B, and 1C received supplemental water treatments, and the other three boxes served as water controls (no water added). One box in each set $(2 A, 1 B)$ served as animal controls (no animals introduced). Food and water were provided liberally on a regular basis.

Supplemental precipitation was added with a rainulator to one set of the lysimeters (1A, 1B, IC) during July, August, and September of 1988. Water was applied at an intensity of $2.5 \mathrm{in} / \mathrm{h}$ for $13 \mathrm{~min}$, which is the equivalent of the Hanford Site 100-yr storm. This has the effect of adding approximately $0.55 \mathrm{in}$. of water in $13 \mathrm{~min}$. Rain gages were set out to measure how much actual water was added during these treatments. The three water treatments adcied $1.89 \mathrm{in}$. of total water during this test. One of these additional water treatments was witnessed by a local television station and later aired on the local news.

At the end of the test period, the animals were removed and the lysimeters were 1ifted out and disassembled by the 1100 Area rigging crew. Table 2 shows soil moisture samples that were collected again at 6 -in. intervals. All excavated soil was collected and measured before the burrow systems were excavated and mapped (Table 3 ). 


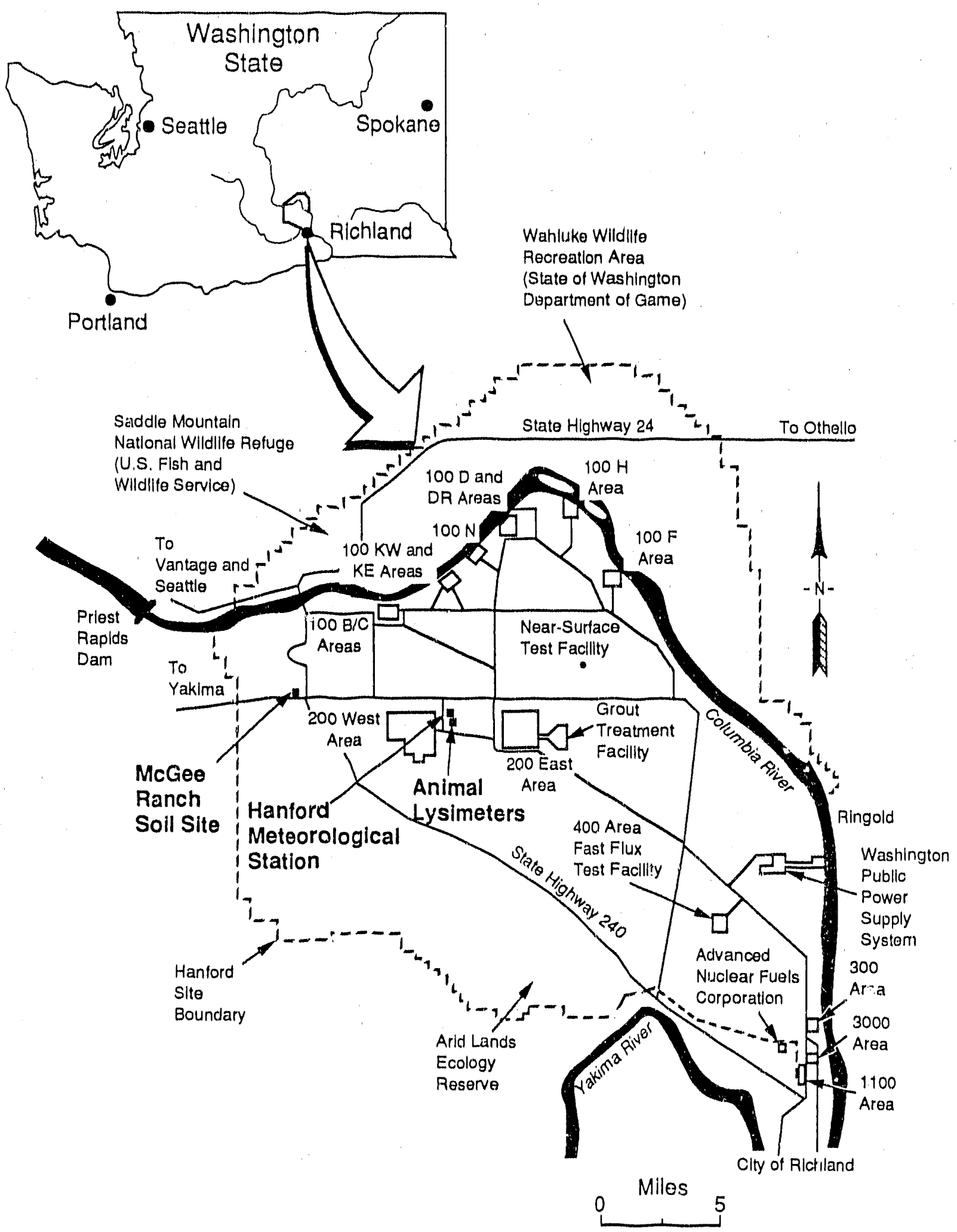

H9007012.1

Figure 3. Location of the McGee Ranch Soil Site. 
Table 1. Soil Moisture Samples Collected April 13 and 14, 1988, at the Installation. (Sheet 1 of 2)

\begin{tabular}{|c|c|c|c|c|c|c|c|}
\hline $\begin{array}{c}\text { Box } \\
\text { number }\end{array}$ & $\begin{array}{l}\text { Depth } \\
\text { (in.) }\end{array}$ & $\begin{array}{c}\text { Can } \\
\text { number }\end{array}$ & $\begin{array}{c}\text { Tare } \\
\text { weight }(\mathrm{g})\end{array}$ & $\begin{array}{c}\text { Wet } \\
\text { weight (g) }\end{array}$ & $\begin{array}{c}\text { Dry } \\
\text { weight }(g)\end{array}$ & $\begin{array}{l}\text { Moisture } \\
\text { (c) }\end{array}$ & $\begin{array}{c}\text { Moisture } \\
(\%)\end{array}$ \\
\hline $1 \mathrm{~A}$ & .59 .00 & 170 & 45.57 & 254.30 & 219.50 & 34.80 & 16.67 \\
\hline $1 \mathrm{~A}$ & 50.50 & 317 & 45.26 & 222.90 & 193.30 & 29.60 & 16.66 \\
\hline $1 \mathrm{~A}$ & 45.50 & 190 & 45.42 & 236.50 & 204.10 & 32.40 & 16.96 \\
\hline $1 A$ & 40.25 & 173 & 44.96 & 192.90 & 168.10 & 24.80 & 16.76 \\
\hline $1 A$ & 34.50 & 304 & 45.06 & 242.80 & 210.80 & 32.00 & 16.18 \\
\hline $1 \mathrm{~A}$ & 29.00 & 268 & 45.73 & 246.10 & 210.80 & 35.30 & 17.62 \\
\hline $1 \mathrm{~A}$ & 24.00 & 243 & 42.98 & 236.80 & 206.10 & 30.70 & 15.84 \\
\hline$\therefore A$ & 19.00 & $81 \mathrm{~A}$ & 45.80 & 203.50 & 178.70 & 24.80 & 15.73 \\
\hline $1 \mathrm{~A}$ & 13.00 & 86 & 43.86 & 275.40 & 237.10 & 38.30 & 16.54 \\
\hline Average & & & & & & & 16.55 \\
\hline $1 B$ & 57.00 & 216 & 43.79 & 197.40 & 174.70 & 22.70 & 14.78 \\
\hline $1 B$ & 51.00 & 67 & 45.99 & 307.60 & 274.30 & 36.30 & 13.88 \\
\hline $1 B$ & 45.00 & 9 & 45.70 & 326.70 & 285.30 & 41.40 & 14.73 \\
\hline $1 B$ & 36.00 & 146 & 45.77 & 257.70 & 221.00 & 36.70 & 17.32 \\
\hline $1 \mathrm{~B}$ & 31.50 & 164 & 45.47 & 258.60 & 225.70 & 32.90 & 15.44 \\
\hline $1 B$ & 26.25 & 321 & 45.58 & 248.70 & 218.10 & 30.60 & 15.06 \\
\hline $1 B$ & 17.50 & 275 & 45.91 & 225.70 & 202.60 & 23.10 & 12.85 \\
\hline $1 B$ & 13.00 & 68 & 46.27 & 227.90 & 202.20 & 25.70 & 14.15 \\
\hline Average & & & & & & & 14.78 \\
\hline $1 C$ & 58.50 & 265 & 45.73 & 236.20 & 206.90 & 29.30 & 15.38 \\
\hline $1 C$ & 52.00 & 299 & 45.52 & 267.70 & 230.70 & 37.00 & 16.66 \\
\hline $1 C$ & 44.50 & 215 & $43.4^{\prime}$ & 256.70 & 222.80 & 33.90 & 15.89 \\
\hline $1 C$ & 37.50 & 220 & 44.16 & 246.50 & 214.40 & 32.10 & 15.86 \\
\hline $1 C$ & 31.00 & 165 & 44.81 & 198.50 & 175.60 & 22.90 & 14.90 \\
\hline $1 C$ & 22.50 & 207 & 42.33 & 227.70 & 202.10 & 25.60 & 13.81 \\
\hline $1 C$ & 16.50 & 73 & 43.66 & 247.80 & 221.70 & 26.10 & 12.79 \\
\hline $1 C$ & 13.00 & 415 & 47.00 & 220.40 & 198.10 & 22.30 & 12.86 \\
\hline Average & & & & & & & 14.77 \\
\hline
\end{tabular}


Table 1. Soil Moisture Samples Collected April 13 and 14, 1988, at the Installation. (Sheet 2 of 2)

\begin{tabular}{|c|c|c|c|c|c|c|c|}
\hline $\begin{array}{c}\text { Box } \\
\text { number }\end{array}$ & $\begin{array}{l}\text { Depth } \\
\text { (in.) }\end{array}$ & $\begin{array}{c}\text { Can } \\
\text { number }\end{array}$ & $\begin{array}{c}\text { Tare } \\
\text { weight (g) }\end{array}$ & $\begin{array}{c}\text { Wet } \\
\text { weight }(\mathrm{g})\end{array}$ & $\begin{array}{c}\text { Dry } \\
\text { weight (g) }\end{array}$ & $\begin{array}{l}\text { Moisture } \\
\text { (g) }\end{array}$ & $\begin{array}{c}\text { Moisture } \\
(\%)\end{array}$ \\
\hline $2 A$ & 53.50 & 53 & 45.80 & 241.40 & 217.50 & 23.90 & 12.22 \\
\hline $2 A$ & 45.25 & 280 & 45.45 & 240.80 & 218.40 & 22.40 & 11.47 \\
\hline $2 \mathrm{~A}$ & 38.00 & 272 & 44.77 & 232.40 & 205.20 & 27.20 & 14.50 \\
\hline $2 \mathrm{~A}$ & 31.75 & 328 & 45.59 & 217.40 & 193.50 & 23.90 & 13.91 \\
\hline $2 A$ & 25.50 & 201 & 44.38 & 223.70 & 195.60 & 28.10 & 15.67 \\
\hline $2 A$ & 19.75 & 33 & 45.20 & 263.00 & 233.00 & 30.00 & 13.77 \\
\hline $2 A$ & 13.00 & 51 & 44.80 & 214.30 & 187.60 & 26.70 & 15.75 \\
\hline Average & & & & & & 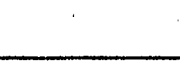 & 13.90 \\
\hline $2 B$ & 54.00 & 25 & 45.70 & 248.10 & 223.00 & 25.10 & 12.40 \\
\hline $2 B$ & 45.50 & 126 & 45.60 & 238.50 & 215.80 & 22.70 & 11.77 \\
\hline $2 B$ & 39.75 & 261 & 45.44 & 280.60 & 249.20 & 31.40 & 13.35 \\
\hline $2 B$ & 33.50 & 189. & 43.10 & 255.90 & 228.30 & 27.60 & 12.97 \\
\hline $2 B$ & 27.50 & 288 & 43.59 & 220.20 & 194.50 & 25.70 & 14.55 \\
\hline $2 B$ & 20.50 & 105 & 46.03 & 217.20 & 191.90 & 25.30 & 14.78 \\
\hline $2 B$ & 12.00 & 282 & 45.57 & 268.20 & 235.30 & 32.90 & 14.78 \\
\hline Average & & & & & & & 13.51 \\
\hline $2 C$ & 58.50 & 329 & 45.30 & 216.10 & 193.90 & 22.20 & 13.00 \\
\hline $2 C$ & 51.50 & 271 & 44.21 & 236.60 & 213.40 & 23.20 & 12.06 \\
\hline $2 C$ & 43.00 & $7 A$ & 45.50 & 250.10 & 225.20 & 24.90 & 12.17 \\
\hline $2 \mathrm{C}$ & 3.8 .50 & 192 & 45.45 & 255.60 & 230.40 & 25.20 & 11.99 \\
\hline $2 C$ & 33.00 & 10 & 45.47 & 204.40 & 184.20 & 20.20 & 12.71 \\
\hline $2 C$ & 26.00 & 111 & 43.50 & 234.20 & 214.00 & 20.20 & 10.59 \\
\hline $2 \mathrm{C}$ & 21.00 & 226 & 45.17 & 195.40 & 178.10 & 17.30 & 11.52 \\
\hline $2 \mathrm{C}$ & 12.00 & 14 & 46.65 & 232.90 & 209.10 & 23.80 & 12.78 \\
\hline Average & & & & & & & 12.10 \\
\hline
\end{tabular}




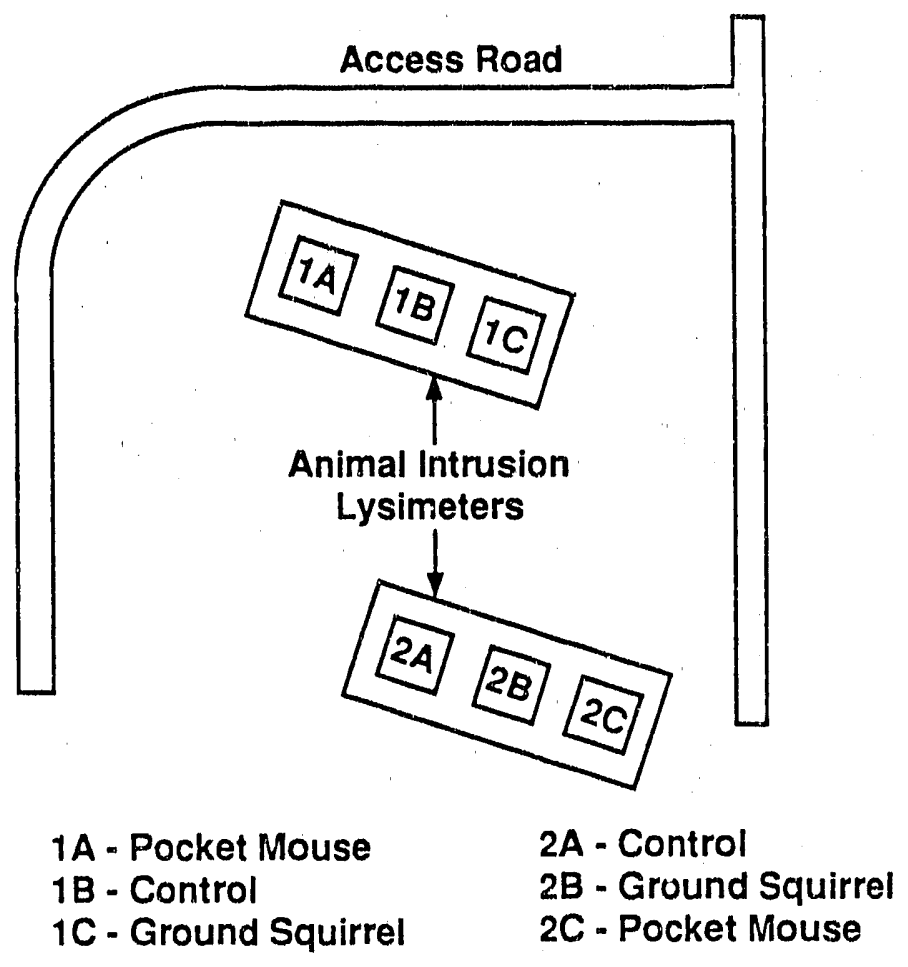

H9007012.3

Figure 4. First Animal Intrusion Lysimeter Configuration. 
Table 2. Soll Moisture Samples Collected in October 1988 at the Completion of Test 1. (Sheet 1 of 2)

\begin{tabular}{|c|c|c|c|c|c|c|c|}
\hline $\begin{array}{c}\text { Box } \\
\text { number }\end{array}$ & $\begin{array}{l}\text { Depth } \\
\text { (in.) }\end{array}$ & $\begin{array}{c}\text { Can } \\
\text { number }\end{array}$ & $\begin{array}{c}\text { Tare } \\
\text { weight (g) }\end{array}$ & $\begin{array}{c}\text { Wet } \\
\text { weight (g) }\end{array}$ & $\begin{array}{c}\text { Dry } \\
\text { weight (g) }\end{array}$ & $\begin{array}{l}\text { Moisture } \\
\text { (g) }\end{array}$ & $\begin{array}{c}\text { Moisture } \\
(\%)\end{array}$ \\
\hline iA & $0-6$ & 51 & 44.80 & 125.50 & 120.30 & 5.20 & 6.44 \\
\hline $1 A$ & $6-12$ & 10 & 45.40 & 120.60 & 114.60 & 6.00 & 7.98 \\
\hline $1 \mathrm{~A}$ & $12-18$ & 26 & 46.20 & 137.80 & $\$ 30.00$ & 7.80 & 8.52 \\
\hline IA & $18-24$ & 280 & 45.50 & 104.50 & 99.40 & 5.10 & 8.64 \\
\hline $1 \mathrm{~A}$ & $24-30$ & 47 & 45.40 & 145.10 & 135.30 & 9.80 & 9.83 \\
\hline $1 A$ & $30-36$ & 275 & 46.00 & 125.20 & 117.40 & 7.80 & 9.85 \\
\hline $1 \mathrm{~A}$ & $36-42$ & 63 & 43.70 & 139.60 & 130.30 & 9.30 & 9.70 \\
\hline $1 A$ & $42-48$ & 65 & 45.50 & 138.20 & 129.60 & 8.60 & 9.28 \\
\hline Average & & & & & & & 8.78 \\
\hline $1 B$ & $0-6$ & 329 & 45.30 & 115.20 & 110.10 & 5.10 & 7.30 \\
\hline $1 B$ & $6-12$ & 157 & 44.60 & 146.50 & 137.70 & 8.80 & 8.64 \\
\hline $1 B$ & $12-20$ & 105 & 46.00 & 137.10 & 128.00 & 9.10 & 9.99 \\
\hline $1 B$ & $20-28$ & 173 & 44.90 & 149.30 & 138.30 & 11.00 & 10.54 \\
\hline $1 B$ & $28-36$ & 333 & 45.40 & 138.90 & 128.60 & 10.30 & 11.02 \\
\hline $1 \mathrm{~B}$ & $36-44$ & 312 & 45.60 & 155.70 & 143.50 & 12.20 & 11.08 \\
\hline $1 \mathrm{~B}$ & $44-48$ & 9 & 45.80 & 133.60 & 124.60 & 9.00 & 10.25 \\
\hline Average & & & & & & & 9.83 \\
\hline $1 C$ & $0-6$ & 226 & 45.30 & 132.70 & 125.80 & 6.90 & 7.89 \\
\hline $1 C$ & $6-12$ & 277 & 45.40 & 140.90 & 132.30 & 8.60 & 9.01 \\
\hline $1 C$ & $12-18$ & 274 & 45.30 & 137.50 & 128.80 & 8.70 & 9.44 \\
\hline $1 C$ & $18-24$ & 42 & 45.70 & 133.30 & 124.30 & 9.00 & 10.27 \\
\hline $1 C$ & $24-30$ & 11 & 46.40 & 136.40 & 126.60 & 9.80 & 10.89 \\
\hline $1 C$ & $30-36$ & 124 & 45.60 & 141.60 & 131.10 & 10.50 & 10.94 \\
\hline $1 C$ & $36-42$ & 192 & 45.50 & 117.20 & 109.70 & 7.50 & 10.46 \\
\hline $1 C$ & $42-48$ & 299 & 45.60 & 110.20 & 103.90 & 6.30 & 9.75 \\
\hline Average & & & & & & & 9.83 \\
\hline
\end{tabular}


Table 2. Soil Moisture Samples Collected in October 1988 at the Completion of Test 1 . (Sheet 2 of 2)

\begin{tabular}{|c|c|c|c|c|c|c|c|}
\hline $\begin{array}{c}\text { Box } \\
\text { number }\end{array}$ & $\begin{array}{l}\text { Depth } \\
\text { (in.) }\end{array}$ & $\begin{array}{c}\text { Can } \\
\text { number }\end{array}$ & $\begin{array}{c}\text { Tare } \\
\text { weight }(g)\end{array}$ & $\begin{array}{c}\text { Wet } \\
\text { weight }(g)\end{array}$ & $\begin{array}{c}\text { Dry } \\
\text { weight }(g)\end{array}$ & $\begin{array}{l}\text { Moisture } \\
\text { (g) }\end{array}$ & $\begin{array}{c}\text { Moisture } \\
(\%)\end{array}$ \\
\hline $2 A$ & $0-6$ & 73 & 43.80 & 127.50 & 123.00 & 4.50 & 5.38 \\
\hline $2 A$ & $6-12$ & 117 & 44.00 & 119.70 & 113.90 & 5.80 & 7.66 \\
\hline $2 \mathrm{~A}$ & $12-18$ & 271 & 44.30 & 142.80 & 134.30 & 8.50 & 8.63 \\
\hline $2 A$ & $18-24$ & 33 & 45.20 & 123.80 & 116.70 & 7.10 & 9.03 \\
\hline $2 A$ & $24-30$ & 25 & 45.80 & 120.40 & 113.20 & 7.20 & 9.65 \\
\hline $2 A$ & $30-36$ & 328 & 45.60 & 130.20 & 122.30 & 7.90 & 9.34 \\
\hline $2 A$ & $36-42$ & 268 & 45.80 & 99.50 & 94.80 & 4.70 & 8.75 \\
\hline $2 A$ & $42-48$ & $7 A$ & 45.60 & 85.70 & 82.70 & 3.00 & 7.48 \\
\hline Average & & & & & & & 8.24 \\
\hline $2 B$ & $0-6$ & 717 & 47.10 & 125.10 & 120.60 & 4.50 & 5.77 \\
\hline $2 B$ & $6-12$ & 709 & 47.40 & 157.60 & 149.30 & 8.30 & 7.53 \\
\hline $2 B$ & $12-18$ & 710 & 47.70 & 140.00 & 131.80 & 8.20 & 8.88 \\
\hline $2 \mathrm{~B}$ & $18-24$ & 706 & 47.30 & 139.70 & 131.00 & 8.70 & 9.42 \\
\hline $2 B$ & $24-30$ & 707 & 46.90 & 131.50 & 123.60 & 7.90 & 9.34 \\
\hline $2 B$ & $30-36$ & 712 & 48.00 & 126.70 & $120: 10$ & 6.60 & 8.39 \\
\hline $2 B$ & $36-42$ & 713 & 47.60 & 113.20 & 107.30 & 5.90 & 8.99 \\
\hline $2 A$ & $42-48$ & 700 & 48.10 & 93.70 & 90.20 & 3.50 & 7.68 \\
\hline Average & & & & & & & 8.25 \\
\hline $2 \mathrm{C}$ & $0-6$ & 716 & 48.10 & 118.40 & 112.90 & 5.50 & 7.82 \\
\hline $2 \mathrm{C}$ & $6-12$ & 702 & 46.90 & 98.60 & 96.20 & 2.40 & 4.64 \\
\hline $2 C$ & $12-18$ & 715 & 48.80 & 124.90 & 118.50 & 6.40 & 8.41 \\
\hline $2 C$ & $18-24$ & 714 & 48.60 & 143.30 & 134.60 & 8.70 & 9.19 \\
\hline $2 C$ & $24-30$ & 703 & 47.30 & 126.50 & 118.90 & 7.60 & 9.60 \\
\hline $2 C$ & $30-36$ & 701 & 48.10 & 129.90 & 121.90 & 8.00 & 9.78 \\
\hline $2 C$ & $36-42$ & 705 & 48.00 & 116.50 & 109.90 & 6.60 & 9.64 \\
\hline $2 \mathrm{C}$ & $42-48$ & 704 & 47.40 & 69.20 & 67.50 & 1.70 & 7.80 \\
\hline Average & & & & & & & 8.36 \\
\hline
\end{tabular}


Table 3. Amount of Excavated Soil collected

at the End of Tests 1 and 2 in the

Animal Intrusion Lysimeters.

\begin{tabular}{llll}
\hline $\begin{array}{c}\text { Box } \\
\text { number }\end{array}$ & Test & \multicolumn{1}{c}{ Species } & $\begin{array}{c}\text { Soil collected } \\
(\mathrm{L})\end{array}$ \\
\hline $1 A$ & 1 & Pocket mouse & 22 \\
$1 \mathrm{C}$ & 1 & Ground squirre & 13.5 \\
$2 B$ & 1 & Ground squirre & 11.5 \\
$2 C$ & 1 & Pocket mouse & 34 \\
$1 A$ & 2 & Pocket gopher & 22.5 \\
$1 C$ & 2 & Pocket mouse & 22.6 \\
$2 A$ & 2 & Pocket gopher & 157.5 \\
$2 C$ & 2 & Pocket gopher & 90 \\
\hline
\end{tabular}

\subsection{RESULTS}

Information collected from the first treatment combination using pocket mice and Townsend ground squirrels indicates that the $1.8 \mathrm{in}$. of additional precipitation, which was primarily applied with the rainulator, was removed from the lysimeters that contained burrows. The lysimeters with animal burrows ended up with soil moisture levels almost identical to the controls (no additional precipitation). This is illustrated in Figure 5, which compares a pocket mouse plot and a control plot that both received additional precipitation with the rainulator. The pocket mouse plot started with $16.55 \%$ moisture and ended up with $8.78 \%$ moisture (a net loss of $7.8 \%$ ). The control plot started with $14.78 \%$ moisture and ended up with $9.83 \%$ (a net loss of $4.95 \%$ ). The pocket mouse plot 1 ost $3 \%$ more moisture than the control.

Appendix A contains the soil-conductance probe data that were collected. The graphs that have been generated from these data are presented in Appendix B.

\subsection{SUMMARY OF TEST 1}

Information collected from this first test (conducted during the summer) indicates that plots containing animals lost more water than did the control plots that did not contain animals. Mechanisms that might account for this include evaporation loss caused by continuous soil turnover as a result of burrowing activities, and moisture loss from open burrows, which allow some air circulation. 


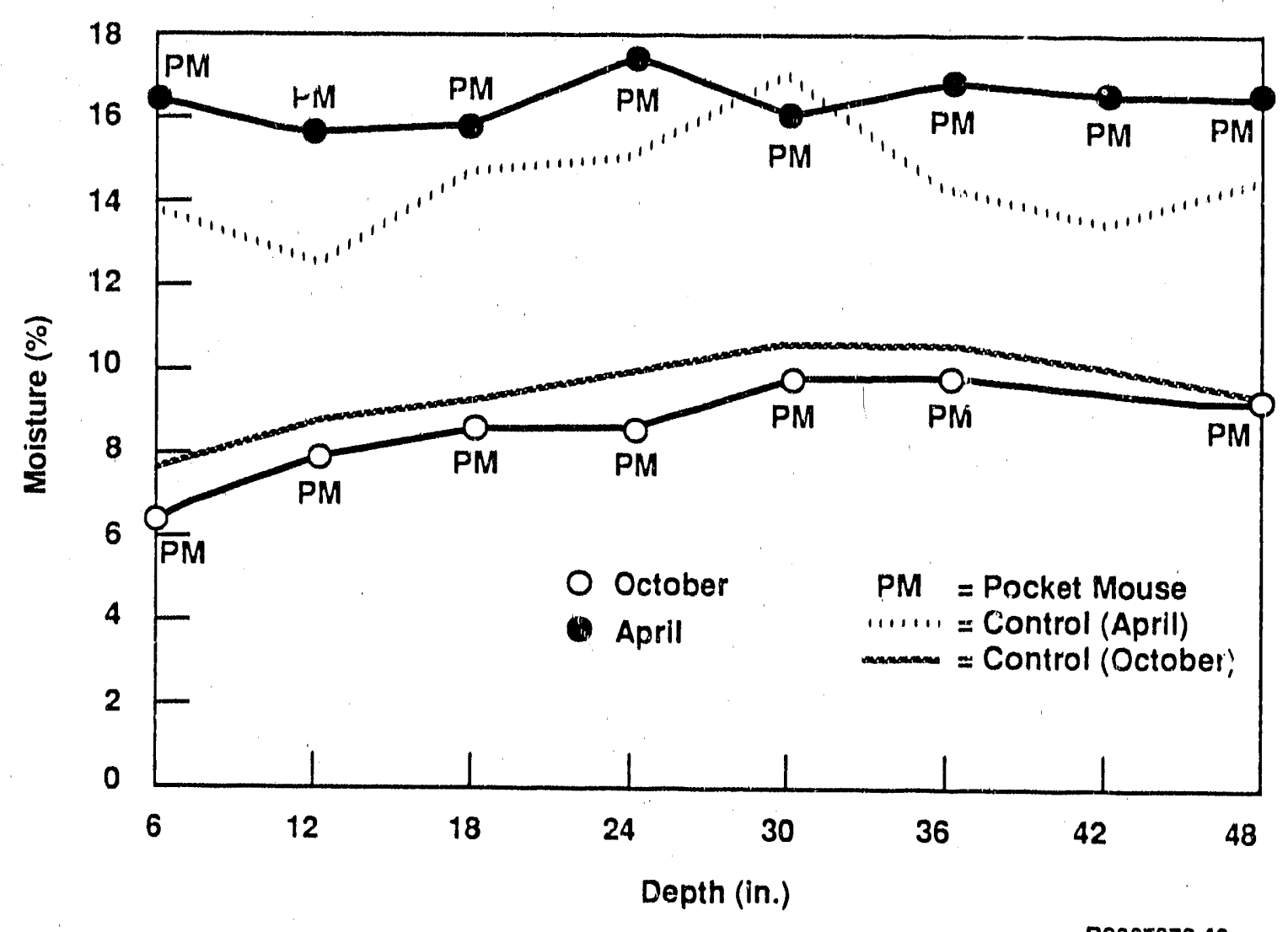

Figure 5. Soil Moisture Profile with Rainulator.

\subsection{TEST 2}

The second test, using three pocket gophers (Thomomys taipoides) and one Great Basin pocket mouse (Perognathus parvus), was started in mid-November 1988 (Figure 6). Only one pocket mouse was captured because of inclement weather, so a pocket gopher was used instead. The lys imeters were again lined with a 28-mil plastic liner, filled with 6 to 8 in. of pea grave1, a geotextile, and $4 \mathrm{ft}$ of McGee Ranch soil. In this test, two access ports per lysimeter were installed so that a hydroprobe could be used to take more accurate soil moisture measurements. Soil moisture samples were collected when the access tubes were installed (Table 4). Frozen soil conditions and frozen water lines during this particular test prevented the addition of supplemental water. However, $4.14 \mathrm{in}$. of supplemental moisture was added in the form of natural precipitation (rain and snow) that, according to the Hanford Meteorological Station, was $178 \%$ of normal by the end of March.

The lysimeters were pulled out and disassembled in early Apri1, The excavated surface soil was collected and measured (Table 3) and the burrow systems were excavated and mapped. Soil moisture samples were again collected at 6 -in. intervals (Table 5). 


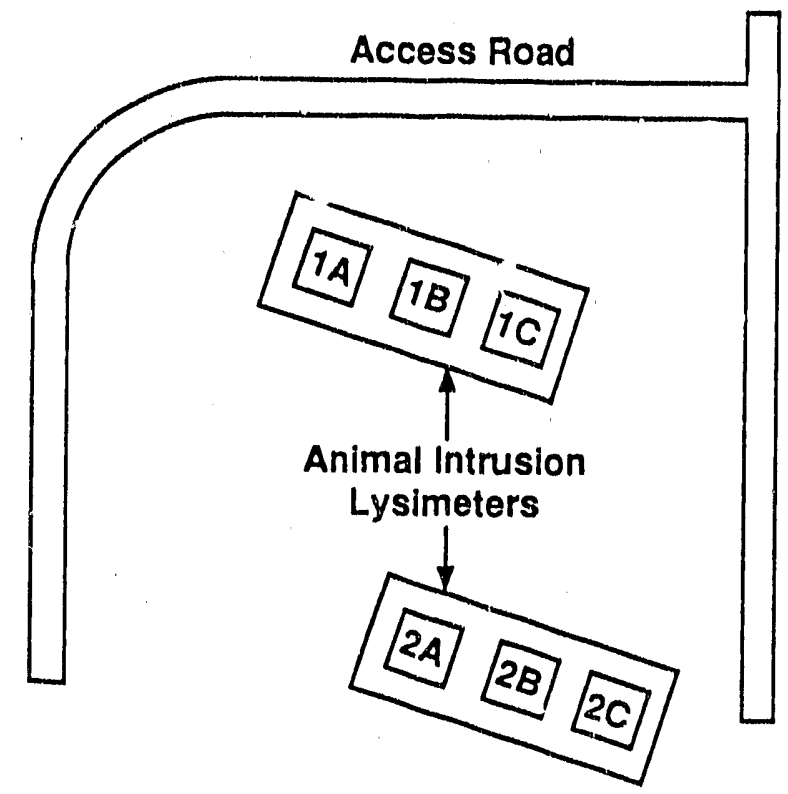

\begin{abstract}
1 A - Pocket Gopher
1B - Control

2A - Pocket Gopher

1C - Pocket Mouse

2B - Control

2C - Pocket Gopher
\end{abstract}

H9007012.2

Figure 6. Second Animal Intrusion Lysimeter Configuration. 
Table 4. Soll Moisture Samples Collected November 18, 1988, at the Installation of the Hydroprobe in Test 2. (Sheet 1 of 4)

\begin{tabular}{|c|c|c|c|c|c|c|c|}
\hline $\begin{array}{c}\text { Box } \\
\text { number }\end{array}$ & $\begin{array}{l}\text { Depth } \\
\text { (in.) }\end{array}$ & $\begin{array}{c}\text { Can } \\
\text { number }\end{array}$ & $\begin{array}{c}\text { Tare } \\
\text { weight (g) }\end{array}$ & $\begin{array}{c}\text { Wet } \\
\text { weight }(g)\end{array}$ & $\begin{array}{c}\text { Dry } \\
\text { weight (g) }\end{array}$ & $\begin{array}{l}\text { Moisture } \\
\text { (g) }\end{array}$ & $\begin{array}{c}\text { Moisture } \\
(\%)\end{array}$ \\
\hline$|A-1|$ & 2 & 234 & 44.90 & 165.20 & 151.80 & 13.40 & 11.14 \\
\hline$|A-1|$ & 6 & 226 & 45.30 & 181.60 & 168.80 & 12.80 & 9.39 \\
\hline$|A-1|$ & $1 ?$ & 65 & 45.50 & 172.40 & 159.30 & 13.10 & 10.32 \\
\hline$|A-1|$ & 18 & 708 & 47.40 & 197.90 & 183.50 & 14.40 & 9.57 \\
\hline$|A-1|$ & 24 & 86 & 43.80 & 168.90 & 157.90 & 11.00 & 8.79 \\
\hline $1 A-11$ & 30 & 709 & 47.40 & 190.40 & 178.10 & 12.30 & 8.60 \\
\hline $1 A-11$ & 36 & 710 & 47.70 & 210.60 & 195.60 & 15.00 & 9.21 \\
\hline $1 A-11$ & 42 & 25 & 45.130 & 158.60 & 148.10 & 10.50 & 9.31 \\
\hline $1 \mathrm{~A}-11$ & 48 & 716 & 48.10 & 224.80 & 209.20 & 15.60 & 8.83 \\
\hline Average & & & & & & & 9.46 \\
\hline $1 A-12$ & 2 & 703 & 47.30 & 249.80 & 227.20 & 22.60 & 11.16 \\
\hline $1 . A-12$ & 6 & 702 & 46.90 & 247.30 & 227.40 & 19.90 & 9.93 \\
\hline $1 A-12$ & 12 & 715 & 48.80 & 251.20 & 231.90 & 19.30 & 9.54 \\
\hline $1 A-12$ & 18 & 706 & 47.30 & 207.20 & 193.30 & 13.90 & 8.69 \\
\hline $1 A-12$ & 24 & 713 & 47.60 & 175.40 & 164.40 & 11.00 & 8.61 \\
\hline $1 A-12$ & 30 & 274 & 45.30 & 148.80 & 141.10 & 7.70 & 7.44 \\
\hline $1 A-12$ & 36 & 86 & 43.80 & 156.40 & 147.00 & 9.40 & 8.35 \\
\hline $1 A-12$ & 42 & 277 & 45.40 & 170.20 & 158.90 & 11.30 & 9.05 \\
\hline $1 A-12$ & 48 & 25 & 45.80 & 186.80 & 175.30 & 11.50 & 8.16 \\
\hline Average & & & & & & & 8.99 \\
\hline $\begin{array}{l}\text { Total } \\
\text { Average } \\
\text { Box } 1 \mathrm{~A}\end{array}$ & & & & & & & 9.23 \\
\hline $1 B-13$ & 2 & 11 & 46.40 & 167.70 & 153.80 & 13.90 & 11.46 \\
\hline $1 \mathrm{~B}-13$ & 6 & 707 & 46.90 & 222.30 & 204.40 & 17.90 & 10.21 \\
\hline $18-13$ & 12 & 708 & 47.40 & 223.60 & 207.70 & 15.90 & 9.02 \\
\hline $18 \cdot 13$ & 18 & 715 & 48.80 & 214.70 & 200.10 & 14.60 & 8.80 \\
\hline $1 B-13$ & 24 & 710 & 47.70 & 228.00 & 211.70 & 16.30 & 9.04 \\
\hline $18-13$ & 30 & 33 & 45.20 & 182.00 & 169.20 & 12.80 & 9.36 \\
\hline $1 \mathrm{~B}-13$ & 36 & 124 & 45.60 & 165.40 & 154.60 & 10.80 & 9.02 \\
\hline $1 \mathrm{~B} \cdot 13$ & 42 & 328 & 45.60 & 174.90 & 163.00 & 11.90 & 9.20 \\
\hline $1 \mathrm{~B}-13$ & 48 & 700 & 48.10 & 237.30 & 220.90 & 16.40 & 8.67 \\
\hline Average & & & & & & & 9.42 \\
\hline
\end{tabular}


Table 4. Soll Moisture Samples Collected November 18, 1988, at the Installation of the Hydroprobe in rest 2. (Sheet 2 of 4)

\begin{tabular}{|c|c|c|c|c|c|c|c|}
\hline $\begin{array}{c}\text { Box } \\
\text { number }\end{array}$ & $\begin{array}{l}\text { Depth } \\
\text { (in.) }\end{array}$ & $\begin{array}{c}\text { Can } \\
\text { number }\end{array}$ & $\begin{array}{c}\text { Tare } \\
\text { weight }(\mathrm{g})\end{array}$ & $\begin{array}{c}\text { Wet } \\
\text { weight (g) }\end{array}$ & $\begin{array}{c}\text { Dry } \\
\text { weight (g) }\end{array}$ & $\begin{array}{l}\text { Moisture } \\
\text { (g) }\end{array}$ & $\begin{array}{l}\text { Moisture } \\
(\%)\end{array}$ \\
\hline $1 \mathrm{~B}-14$ & 2 & 117 & 44.00 & 175.40 & 161.50 & 13.90 & 10.58 \\
\hline $18-14$ & 6 & 271 & 44.30 & 186.10 & 176.30 & 9.80 & 6.91 \\
\hline $18-14$ & 12 & 234 & 44.90 & 162.80 & 152.70 & 10.10 & 8.57 \\
\hline$\sqrt[1 B .14]{14}$ & 18 & 703 & 47.30 & 248.90 & 230.20 & 18.70 & 9.28 \\
\hline $1 \mathrm{~B} \cdot 14$ & 24 & 63 & 43.70 & 176.30 & 164.80 & 11.50 & 8.67 \\
\hline $18-14$ & 30 & 73 & 43.80 & 190.50 & 180.90 & 9.60 & 6.54 \\
\hline $18-14$ & 36 & 333 & 45.40 & 172.80 & 162.20 & 10.60 & 8.32 \\
\hline $1 B-14$ & 42 & 714 & 48.60 & 183.80 & 172.90 & 10.90 & 8.06 \\
\hline $1 \mathrm{~B}-14$ & 48 & 704 & 47.40 & 204.30 & 189.90 & 14.40 & 9.18 \\
\hline Average & & & & & & & 8.46 \\
\hline $\begin{array}{c}\text { Total } \\
\text { Average } \\
\text { Box } 1 \mathrm{~B}\end{array}$ & & & & & & & 8.94 \\
\hline $1 C-15$ & 2 & 702 & 46.90 & 273.70 & 250.40 & 23.30 & 10.27 \\
\hline $1 C-15$ & 6 & 105 & 46.00 & 158.90 & 149.50 & 9.40 & 8.33 \\
\hline $1 C-15$ & 12 & 111 & 43.60 & 167.20 & 156.40 & 10.80 & 8.74 \\
\hline $1 C-15$ & 18 & $7 A$ & 45.60 & 173.50 & 162.50 & 11.00 & 8.60 \\
\hline $1 C-15$ & 24 & 706 & 47.30 & 227.30 & 211.50 & 16.30 & 9.03 \\
\hline $1 C-15$ & 30 & 173 & 44.90 & 155.20 & 144.70 & 10.50 & 9.52 \\
\hline $1 C-15$ & 36 & 709 & 47.40 & 216.30 & 199.10 & 17.20 & 17.18 \\
\hline $1 C-15$ & 42 & 192 & 45.50 & 156.80 & 147.20 & 9.60 & 8.63 \\
\hline $1 C \cdot 15$ & 48 & 51 & 44.80 & 177.10 & 165.10 & 12.00 & 9.07 \\
\hline Average & & & & & & & 9.15 \\
\hline $1 C-16$ & 2 & 10 & 45.40 & 174.50 & 161.60 & 12.90 & 9.99 \\
\hline $16-16$ & 6 & 716 & 48.10 & 229.40 & 216.40 & 13.00 & 7.17 \\
\hline $1 C-16$ & 12 & 712 & 48.00 & 226.70 & 211.80 & 14.90 & 8.34 \\
\hline $1 C .16$ & 18 & 47 & 45.40 & 163.60 & 153.70 & 9.90 & 8.38 \\
\hline $1 C-16$ & 24 & 329 & 45.30 & 166.70 & 156.40 & 10.30 & 8.48 \\
\hline $1 C-16$ & 30 & 701 & 48.10 & 219.80 & 204.50 & 15.30 & 8.91 \\
\hline $1 \mathrm{C}-16$ & 36 & 717 & 47.10 & 242.40 & 223.90 & 18.50 & 9.47 \\
\hline $1 C-16$ & 42 & 705 & 48.00 & 266.30 & 246.90 & 19.40 & 8.89 \\
\hline $1 \mathrm{C}-16$ & 48 & 713 & 47.60 & 248.30 & 229.30 & 19.00 & 9.47 \\
\hline Average & & & & & & & 8.79 \\
\hline $\begin{array}{c}\text { Total } \\
\text { Average } \\
\text { Box iC }\end{array}$ & & & & & & & 8.97 \\
\hline
\end{tabular}


Table 4. Sol1 Molsture Samples Collected November 18, 1988, at the Installation of the Hydroprobe in Test 2. (Sheet 3 of 4)

\begin{tabular}{|c|c|c|c|c|c|c|c|}
\hline $\begin{array}{c}\text { Box } \\
\text { numiber }\end{array}$ & $\begin{array}{l}\text { Depth } \\
\text { (in.) }\end{array}$ & $\begin{array}{c}\text { Can } \\
\text { number }\end{array}$ & $\begin{array}{c}\text { Tare } \\
\text { weight }(g)\end{array}$ & $\begin{array}{c}\text { Wet } \\
\text { weight }(g)\end{array}$ & $\begin{array}{c}\text { Dry } \\
\text { weight (g) }\end{array}$ & $\begin{array}{l}\text { A oisture } \\
\text { (g) }\end{array}$ & $\begin{array}{c}\text { Moisture } \\
(\%)\end{array}$ \\
\hline $2 A-21$ & 2 & 26 & 46.20 & 143.20 & 132.30 & 10.90 & 11.24 \\
\hline $2 A-21$ & 6 & 192 & 45.50 & 120.10 & 113.40 & 6.70 & 8.98 \\
\hline $2 A-21$ & 12 & 275 & 46.00 & 132.70 & 125.50 & 7.20 & 8.30 \\
\hline $2 A-21$ & 18 & 10 & 45.40 & 137.30 & 129.00 & 8.30 & 9.03 \\
\hline $2 A-21$ & 24 & 234 & 44.90 & 132.00 & 123.80 & 8.20 & 9.41 \\
\hline $2 A-21$ & 30 & 703 & 47.30 & 127.20 & 12030 & 6.90 & 8.64 \\
\hline $2 A-21$ & 36 & 11 & 46.40 & 122.60 & 116.30 & $6.3 \mathrm{C}$ & 8.27 \\
\hline $2 A-21$ & 42 & 47 & 45.40 & 116.70 & 110.60 & 6.10 & 8.56 \\
\hline $2 A-21$ & 48 & 105 & 46.00 & 127.90 & 120.50 & 7.40 & 9.04 \\
\hline Average & & & & & & & 9.05 \\
\hline $2 A-22$ & 2 & 111 & 43.60 & 127.60 & 918.30 & 9.30 & 11.07 \\
\hline $2 A-22$ & 6 & 333 & 45.40 & 123.20 & 118.40 & 4.80 & 6.17 \\
\hline $2 A-22$ & 12 & 280 & 45.50 & 160.70 & 153.80 & 6.90 & 5.99 \\
\hline $2 A-22$ & 18 & 117 & 44.00 & 160.00 & 156.90 & 3.10 & 2.67 \\
\hline $2 A-22$ & 24 & 73 & 43.80 & 134.90 & 127.70 & 7.20 & 7.90 \\
\hline $2 A-22$ & 30 & 299 & 45.60 & 125.80 & 118.50 & 7.30 & 9.10 \\
\hline $2 A-22$ & 36 & 157 & 44.60 & 136.30 & 130.40 & 5.90 & 6.43 \\
\hline $2 A-22$ & 42 & 329 & 45.30 & 127.20 & 119.80 & 7.40 & 9.04 \\
\hline $2 A-22$ & 48 & 146 & 45.70 & 128.30 & 121.60 & 6.70 & 8.11 \\
\hline Average & & & & & & & 7.39 \\
\hline $\begin{array}{l}\text { Total } \\
\text { Average } \\
\text { Box } 2 A\end{array}$ & & & & & & & 8.22 \\
\hline $2 \mathrm{~B}-23$ & 2 & 9 & 45.80 & 139.80 & 129.30 & 10.50 & 11.17 \\
\hline $2 B-23$ & 6 & 226 & 45.30 & 134.40 & 125.80 & 8.60 & 9.65 \\
\hline $2 B-23$ & 12 & 710 & 47.70 & 167.30 & 156.40 & 10.90 & 9.11 \\
\hline $2 B-23$ & 18 & 707 & 46.90 & 161.60 & 149.60 & 12.00 & 10.46 \\
\hline $28-23$ & 24 & 715 & 48.80 & 155.80 & 144.10 & 11.70 & 10.93 \\
\hline $2 B-23$ & 30 & 25 & 45.80 & 128.50 & 120.30 & 8.20 & 9.92 \\
\hline $2 B-23$ & 36 & 277 & 45.40 & & & 0.00 & 0.00 \\
\hline $28-23$ & 42 & 714 & 48.60 & 151.80 & 142.60 & 9.20 & 8.91 \\
\hline $2 B-23$ & 48 & 51 & 44.80 & 124.70 & 117.60 & 7.10 & 8.89 \\
\hline Average & & . & & & & & 8.78 \\
\hline
\end{tabular}


Table 4. Soll Molsture Samples Collected November 18, 1988, at the Installation of the Hydroprobe in Test 2. (Sheet 4 of 4)

\begin{tabular}{|c|c|c|c|c|c|c|c|}
\hline $\begin{array}{c}\text { Box } \\
\text { number }\end{array}$ & $\begin{array}{c}\text { Depth } \\
(\ln .)\end{array}$ & $\begin{array}{c}\text { Can } \\
\text { number }\end{array}$ & $\begin{array}{c}\text { Tare } \\
\text { weight (g) }\end{array}$ & $\begin{array}{c}\text { Wet } \\
\text { weight (g) }\end{array}$ & $\begin{array}{c}\text { Dry } \\
\text { weight (g) }\end{array}$ & $\begin{array}{l}\text { Moisture } \\
\text { (g) }\end{array}$ & $\begin{array}{c}\text { Molsture } \\
(\%)\end{array}$ \\
\hline $2 B-24$ & 2 & 271 & 44.30 & 138.00 & 127.10 & 10.90 & 11.63 \\
\hline $2 B-24$ & 6 & $7 A$ & 45.60 & 141.10 & 133.90 & 7.20 & 7.54 \\
\hline $28-24$ & 12 & 63 & 43.70 & 124.10 & 120.20 & 3.90 & 4.85 \\
\hline $2 B-24$ & 18 & 173 & 44.90 & 114.40 & 107.70 & 6.70 & 9.64 \\
\hline $28-24$ & 24 & 42 & 45.70 & 127.70 & 119.30 & 8.40 & 10.24 \\
\hline $2 B-24$ & 30 & 712 & 48.00 & 182.10 & 170.20 & 11.90 & 8.87 \\
\hline $2 B \cdot 24$ & 36 & 65 & 45.50 & 123.20 & 116.30 & 6.90 & 8.88 \\
\hline$\angle B-24$ & 42 & 33 & 45.20 & 147.40 & 138.80 & 8.60 & 8.41 \\
\hline $2 B-24$ & 48 & 124 & 45.60 & 138.90 & 131.30 & 7.60 & 8.15 \\
\hline Average & & & & & & & 8.69 \\
\hline $\begin{array}{l}\text { Total } \\
\text { Average } \\
\text { Box 2B }\end{array}$ & & & & & & & 8.74 \\
\hline $2 C-25$ & 2 & 705 & 47.30 & 185.00 & 172.10 & 12.90 & 9.37 \\
\hline $2 C-25$ & 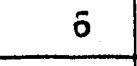 & 704 & 47.40 & 175.90 & 168.10 & 7.80 & 6.07 \\
\hline $2 C-25$ & 12 & 700 & 48.10 & 182.80 & 171.20 & 11.60 & 8.61 \\
\hline $2 C-25$ & 18 & 268 & 45.80 & 170.20 & 160.50 & 9.70 & 7.80 \\
\hline $2 C-25$ & 24 & 117 & 44.00 & 153.10 & 143.00 & 10.10 & 9.26 \\
\hline $2 C-25$ & 30 & 714 & 48.60 & 153.00 & 144.20 & 8.80 & 8.43 \\
\hline $2 C-25$ & 36 & 63 & 43.70 & 176.80 & 163.80 & 13.00 & 9.77 \\
\hline $26 .-25$ & 42. & 711 & 47.20 & 197.50 & 183.20 & 14.30 & 9.51 \\
\hline $2 C-25$ & 48 & 704 & 47.40 & 217.60 & 201.20 & 16.40 & 9.64 \\
\hline Average & & & & & & & 8.72 \\
\hline $2 C-26$ & 2 & 701 & 48.10 & 173.90 & 162.60 & 11.30 & 8.98 \\
\hline $2 C-26$ & 6 & 86 & 43.80 & 135.40 & 129.10 & 6.30 & 6.88 \\
\hline $2 C-26$ & 12 & 274 & 45.30 & 122.90 & 118.10 & 4.80 & 6.19 \\
\hline $2 C .-26$ & 18 & 705 & 48.00 & 166.80 & 156.30 & 10.50 & 8.84 \\
\hline $2 C-26$ & 24 & 713 & 47.60 & 153.00 & 144.30 & 8.70 & 8.25 \\
\hline $2 C-26$ & 30 & 328 & 45.60 & 129.90 & 124.20 & 5.70 & 6.76 \\
\hline $2 C-26$ & 36 & 716 & 48.10 & 181.00 & 170.60 & 10.40 & 7.83 \\
\hline $2 C-26$ & 42 & 717 & 47.10 & 172.60 & 161.70 & 10.90 & 8.69 \\
\hline $2 C-26$ & 48 & 702 & 46.90 & 182.60 & 172.70 & 9.90 & 7.30 \\
\hline Average & & & & & & & 7.75 \\
\hline $\begin{array}{l}\text { Total } \\
\text { Average } \\
\text { Box IC }\end{array}$ & & & & & & & 8.23 \\
\hline
\end{tabular}


Table 5. Sol1 Molsture Samples Collected in Aprll 1989 at the End of Test 2. (Sheet 1 of 2)

\begin{tabular}{|c|c|c|c|c|c|c|c|}
\hline $\begin{array}{c}\text { Box } \\
\text { number }\end{array}$ & $\begin{array}{l}\text { Depth } \\
(\text { In.) }\end{array}$ & $\begin{array}{c}\text { Can } \\
\text { number }\end{array}$ & $\begin{array}{c}\text { Tare } \\
\text { welght (g) }\end{array}$ & $\begin{array}{c}\text { Wet } \\
\text { welght (g) }\end{array}$ & $\begin{array}{c}\text { Dry } \\
\text { weight }(g)\end{array}$ & $\begin{array}{c}\text { Molsture } \\
\text { (g) }\end{array}$ & $\begin{array}{c}\text { Moisture } \\
(\%)\end{array}$ \\
\hline $1 \mathrm{~A}$ & 0.6 & 709 & 0.00 & 0.00 & 0.00 & 0.00 & ERR \\
\hline $1 \mathrm{~A}$ & $6-12$ & 63 & 28.16 & 77.85 & 72.38 & 5.47 & 11.01 \\
\hline $1 A$ & $12-18$ & $7 a$ & 29.61 & 68.25 & 63.87 & 4.38 & 11.34 \\
\hline $1 \mathrm{~A}$ & $18-24$ & 47 & 29.67 & 86.76 & 80.85 & 5.91 & 10.35 \\
\hline $1 \mathrm{~A}$ & $24-30$ & 65 & 29.64 & 78.80 & 72.94 & 5.86 & 11.92 \\
\hline $1 \mathrm{~A}$ & $30-36$ & 51 & 29.17 & 88.50 & 82.15 & 6.35 & 10.70 \\
\hline $1 A$ & $36-42$ & 86 & 28.00 & 80.59 & 75.86 & 4.73 & 8.99 \\
\hline $1 \mathrm{~A}$ & $42-48$ & 117 & 27.63 & 94.82 & 88.91 & 5.91 & 8.80 \\
\hline Average & & & & & & & 10.44 \\
\hline $1 B$ & 0.6 & 716 & 34.71 & 79.81 & 75.81 & 4.00 & 8.87 \\
\hline 18 & $6-12$ & 710 & 34.34 & 101.47 & 94.69 & 6.78 & 10.10 \\
\hline 18 & $12-18$ & 33 & 29.35 & 81.89 & 75.8 & 6.07 & 11.55 \\
\hline 18 & $18-24$ & 715 & 35.59 & 93.92 & 87.65 & 6.27 & 10.75 \\
\hline $1 B$ & $24-30$ & 268 & 29.66 & 90.00 & 83.58 & 6.42 & 10.64 \\
\hline $1 \mathrm{~B}$ & $30-36$ & 708 & 34.25 & 59.66 & 57.55 & 2.11 & 8.30 \\
\hline Average & & & & & & & 10.04 \\
\hline $1 C$ & $0-6$ & 702 & 34.14 & 94.00 & 88.20 & 5.80 & 9.69 \\
\hline $1 C$ & $6-12$ & 274 & 29.18 & 95.58 & 88.34 & 7.24 & 10.90 \\
\hline 15 & $12-18$ & 700 & 34.80 & 95.16 & 87.25 & 7.91 & 13.10 \\
\hline $1 \mathrm{C}$ & $18-24$ & 280 & 29.45 & 93.02 & 85.26 & 7.76 & 12.21 \\
\hline $1 C$ & $24-30$ & 312 & 29.55 & 89.77 & 82.62 & 7.15 & 11.87 \\
\hline $1 C$ & $30-36$ & 714 & 34.92 & 86.60 & 81.17 & 5.43 & 10.51 \\
\hline $1 C$ & $36-42$ & 42. & 29.68 & 70.12 & 65.94 & 4.18 & 10.34 \\
\hline 15 & $42-48$ & 25 & 29.84 & 50.40 & 48.44 & 1.96 & 9.53 \\
\hline Average & & & & & & & 11.02 \\
\hline $2 \mathrm{~A}$ & 0.6 & 11 & 29.85 & 75.76 & 71.41 & 4.3 .5 & 9.48 \\
\hline $2 A$ & $6-12$ & 712 & 34.80 & 102.50 & 95.48 & 7.02 & 10.37 \\
\hline
\end{tabular}


WHC-EP-0299 Draft

Table 5. Soll Molsture Samples Collected In Aprif 1989 at the End of Test 2. (Sheet 2 of 2)

\begin{tabular}{|c|c|c|c|c|c|c|c|}
\hline $\begin{array}{c}\text { Box } \\
\text { number }\end{array}$ & $\begin{array}{l}\text { Depth } \\
(\text { (In.) }\end{array}$ & $\begin{array}{c}\text { Can } \\
\text { number }\end{array}$ & $\begin{array}{c}\text { Tare } \\
\text { weight }(g)\end{array}$ & $\begin{array}{c}\text { Wet } \\
\text { weight (g) }\end{array}$ & $\begin{array}{c}\text { Dry } \\
\text { weight (g) }\end{array}$ & $\begin{array}{l}\text { Moisture } \\
\text { (g) }\end{array}$ & $\begin{array}{c}\text { Moisture } \\
(\%)\end{array}$ \\
\hline $2 A$ & $12 \cdot 18$ & 333 & 29.44 & 79.55 & 73.45 & 6.10 & 12.17 \\
\hline $2 A$ & $18-24$ & 271 & 29.78 & 91.15 & 82.66 & 8.49 & 13.83 \\
\hline $2 A$ & $24-30$ & 717 & 33.75 & 91.34 & 84.33 & 7.01 & 12.17 \\
\hline $2 A$ & $30-36$ & 711 & 34.08 & 101.95 & 94.27 & 7.68 & 11.32 \\
\hline $2 A$ & $36-42$ & 105 & 29.48 & 97.02 & 90.59 & 6.43 & 9.52 \\
\hline $2 A$ & $42-48$ & 713 & 34.02 & 72.40 & 69.12 & 3.28 & 8.55 \\
\hline Average & & & & & & & 10.93 \\
\hline $2 \mathrm{~B}$ & $0-6$ & 111 & 27.36 & 86.30 & 81.90 & 4.40 & 7.47 \\
\hline $2 \mathrm{~B}$ & $6-12$ & 192 & 29.04 & 98.79 & 91.33 & 7.46 & 10.70 \\
\hline $2 B$ & $12-18$ & 21 & 30.16 & 91.25 & 83.64 & 7.61 & 12.46 \\
\hline $2 B$ & $18-24$ & 701 & 34.61 & 92.71 & 85.23 & 7.48 & 12.87 \\
\hline $2 B$ & $24-30$ & 707 & 33.64 & 81.00 & 76.16 & 4.84 & 10.22 \\
\hline $2 B$ & $30-36$ & 277 & 29.21 & 49.40 & 47.19 & 2.21 & 10.95 \\
\hline $2 B$ & $36-42$ & 226 & 29.18 & 72.65 & 68.67 & 3.98 & 9.16 \\
\hline Average & & & & & & & 10.54 \\
\hline $2 C$ & 0.6 & 146 & 29.94 & 87.85 & 82.83 & 5.02 & 8.67 \\
\hline $2 C$ & $6-12$ & 124 & 29.77 & 95.69 & 87.64 & 8.05 & 12.21 \\
\hline $2 \mathrm{C}$ & $12-18$ & 173 & 29.08 & 116.49 & 105.20 & 11.29 & 12.92 \\
\hline $2 C$ & $18-24$ & 73 & 28.02 & 61.53 & 57.27 & 4.26 & 12.71 \\
\hline $2 \mathrm{C}$ & $24 \cdot 30$ & 299 & 29.59 & 102.12 & 94.72 & 7.40 & 10.20 \\
\hline $2 C$ & $30-36$ & 275 & 29.95 & 102.12 & 94.72 & 7.40 & 10.25 \\
\hline $2 C$ & $36-42$ & 703 & 34.59 & 97.28 & 91.39 & 5.89 & 9.40 \\
\hline $2 C$ & 42.48 & 329 & 29.43 & 30.85 & 76.05 & 4.80 & 9.33 \\
\hline Average & & & & & & & 10.71 \\
\hline $1 a$ & mound & 706 & 34.16 & 259.85 & 239.76 & 20.09 & 8.90 \\
\hline ic & mound & 704 & 34.25 & 260.60 & 241.86 & 18.74 & 8.28 \\
\hline $2 a$ & mound & 705 & 34.60 & 259.22 & 238.80 & 20.42 & 9.09 \\
\hline $2 c$ & mound & 234 & 29.20 & 212.20 & 202.87 & 9.33 & 5.10 \\
\hline
\end{tabular}




\subsection{RESULTS}

No detailed statistical analysis of the hydroprobe data has been conducted (Appendix $C$ ), but in general it appears that the soll moisture in the top $2 \mathrm{ft}$ was slightly greater in pllots with animal burrows and that from the 2 -ft level down the control plots were wetter. It was observed that even in the winter time, and in particular in the pocket gopher burrows, freshly excavated soll would dry out by the end of the day.

This test provided a good comparison of soll moisture comparisons under natural precipitation conditions during the winter season. Alt six lysimeters had a net increase in soll moisture (Table 6). The four 1ysimeters that contained animals had an average gain of $2.71 \%$ sol1 moisture, whereas the two control lysimeters had an average increase of $1.42 \%$ sol1 motsture. The largest increases in soll moisture in all the lysimeters occurred from 18 to $30 \mathrm{in}$. With the $18 \mathrm{in}$. depth showing the most change. The least amount of change in soll moisture occurred from the $42-i n$. to $48-i n$. depth. At the 18-in. depth there was an average charige of $3.78 \%$ soil moisture and at the 48-in. depth there was an average change of $0.38 \%$. Figures 7 and 8 illustrate this point by showing initial soil moisture conditions at the beginning and at the end of Test 2 in two of the 1ysimeters. Other figures showing these relationships are contained in Appendix $D$.

The amount of soil excavated varied considerably between lysimeters (see Table 3). The pocket gopher in Box $1 \mathrm{~A}$ excavated $22.5 \mathrm{~L}$ of sol1, while the one in Box $2 A$ excavated $157.5 \mathrm{~L}$ of sol1, which is the highest amount collected thus far.

\subsection{SUMMARY OF TEST 2}

Information collected from the socond test (conducted during the winter) indicates that the lysimeters containing animals gained more moisture than the control plots. The greatest change in moisture gain occurred at the 18-in. depth. Very little change in moisture cortent occurred from the 42 - to 48-in. depths. The hydroprobe data have yet to be analyzed. 
Table 6. Soil Moisture Profile Changes During Test 2. (Sheet 1 of 2)

\begin{tabular}{|c|c|c|c|c|c|}
\hline $\begin{array}{c}\text { Box } \\
\text { number }\end{array}$ & $\begin{array}{l}\text { Depth } \\
\text { (in.) }\end{array}$ & Species & $\begin{array}{c}\text { Initial } \\
\text { moisture } \\
(\%)\end{array}$ & $\begin{array}{c}\text { Ending } \\
\text { moisture } \\
(\%)\end{array}$ & $\begin{array}{r}\text { Gain } \\
(\%)\end{array}$ \\
\hline $1 \mathrm{~A}$ & 6 & P. Gopher & 9.66 & & \\
\hline $1 \mathrm{~A}$ & 12 & & 9.93 & 11.01 & 1.08 \\
\hline $1 \mathrm{~A}$ & 18 & & 9.13 & 11.34 & 2.21 \\
\hline $1 \mathrm{~A}$ & 24 & & 8.70 & 10.35 & 1.65 \\
\hline $1 \mathrm{~A}$ & 30 & & 8.02 & 11.92 & 3.90 \\
\hline $1 \mathrm{~A}$ & 36 & & 8.78 & 10.70 & 1.92 \\
\hline $1 \mathrm{~A}$ & 42 & & 9.18 & 8.99 & -0.19 \\
\hline $1 \mathrm{~A}$ & 48 & & 8.49 & 8.80 & 0.31 \\
\hline Average & & & 8.99 & 10.44 & 1.55 \\
\hline $1 B$ & 6 & Control & 8.56 & 8.87 & 0.31 \\
\hline $1 \mathrm{~B}$ & 12 & & 8.79 & 10.10 & 1.31 \\
\hline $1 \mathrm{~B}$ & 18 & & 9.04 & 11.55 & 2.51 \\
\hline $1 \mathrm{~B}$ & 24 & & 8.85 & 10.75 & 1.90 \\
\hline $1 \mathrm{~B}$ & 30 & & 7.95 & 10.64 & 2.69 \\
\hline $1 \mathrm{~B}$ & 36 & & 8.67 & 8.30 & -0.37 \\
\hline $1 B$ & 42 & & 8.63 & & \\
\hline $1 \mathrm{~B}$ & 48 & & 8.92 & & \\
\hline Average & & & 8.68 & 10.04 & 1.39 \\
\hline $1 \mathrm{C}$ & 6 & P. Mouse & 7.75 & 9.69 & 1.94 \\
\hline $1 C$ & 12 & & 8.54 & 10.90 & 2.36 \\
\hline $1 C$ & 18 & & 8.49 & 13.10 & 4.61 \\
\hline $1 C$ & 24 & & 8.75 & 12.21 & 3.46 \\
\hline $1 C$ & 30 & & 9.21 & 11.87 & 2.66 \\
\hline $1 C$ & 36 & & 9.82 & 10.51 & 0.69 \\
\hline $1 \mathrm{C}$ & 42 & & 8.76 & 10.34 & 1.58 \\
\hline $1 C$ & 48 & & 9.27 & 9.53 & 0.26 \\
\hline Average & & & 8.82 & 11.02 & 2.20 \\
\hline
\end{tabular}


Table 6. Soil Moisture Profile Changes During Test 2. (Sheet 2 of 2)

\begin{tabular}{|c|c|c|c|c|c|}
\hline $\begin{array}{c}\text { Box } \\
\text { number }\end{array}$ & $\begin{array}{l}\text { Depth } \\
\text { (in.) }\end{array}$ & Species & $\begin{array}{l}\text { Initial } \\
\text { moisture } \\
(\%)\end{array}$ & $\begin{array}{c}\text { Ending } \\
\text { moisture } \\
(\%)\end{array}$ & $\begin{array}{c}\text { Gain } \\
(\%)\end{array}$ \\
\hline $2 A$ & 6 & P. Gopher & 7.57 & 9.48 & 1.91 \\
\hline $2 A$ & 12 & & 7.14 & 10.37 & 3.23 \\
\hline $2 A$ & 18 & & 5.85 & 12.17 & 6.32 \\
\hline $2 A$ & 24 & & 8.65 & 13.83 & 5.18 \\
\hline $2 A$ & 30 & & 8.87 & 12.17 & 3.30 \\
\hline $2 A$ & 36 & & 7.55 & 11.32 & 3.97 \\
\hline $2 A$ & 42 & & 8.80 & 9.52 & 0.72 \\
\hline $2 A$ & 48 & & $\{8.57$ & 8.55 & -0.02 \\
\hline Average & & & 7.85 & 10.93 & 3.08 \\
\hline $2 B$ & 6 & Control & 8.59 & 7.47 & -1.12 \\
\hline $2 B$ & 12 & & 6.98 & 10.70 & 3.72 \\
\hline $2 \mathrm{~B}$ & 18 & & 10.05 & 12.46 & 2.41 \\
\hline $2 \mathrm{~B}$ & 24 & & 10.58 & 12.87 & 2.29 \\
\hline $2 \mathrm{~B}$ & 30 & & 9.39 & 10.22 & 0.83 \\
\hline $2 B$ & 36 & & & & \\
\hline $2 \mathrm{~B}$ & 42 & & 8.66 & 9.16 & 0.50 \\
\hline 28 & 48 & & & & \\
\hline Average & & & 9.04 & 10.48 & 1.44 \\
\hline $2 C$ & 6 & P. Gopher & 6.47 & 8.67 & 2.20 \\
\hline $2 C$ & 12 & & 7.40 & 12.21 & 4.81 \\
\hline $2 C$ & 18 & & 8.32 & 12.92 & 4.60 \\
\hline $2 C$ & 24 & & 8.75 & 12.71 & 3.96 \\
\hline $2 C$ & 30 & & 7.59 & 10.20 & 2.61 \\
\hline $2 C$ & 36 & & 8.80 & 10.25 & 1.45 \\
\hline $2 C$ & 42 & & 9.10 & 9.40 & 0.30 \\
\hline $2 C$ & 48 & & 8.47 & 9.33 & 0.86 \\
\hline Average & & & 8.11 & 10.71 & 2.60 \\
\hline
\end{tabular}




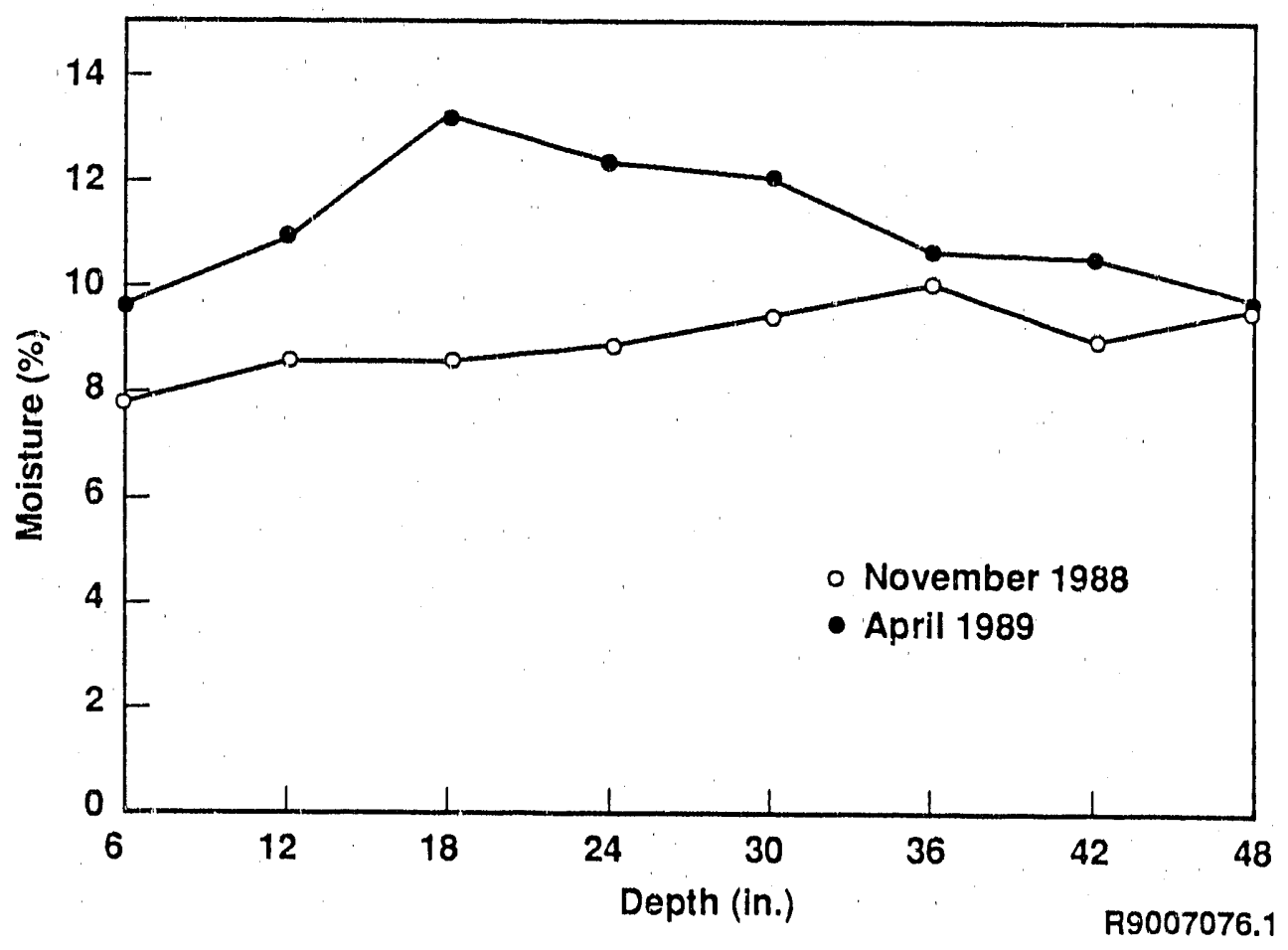

Figure 7. Soil Moisture Profile of the Pocket Mouse.

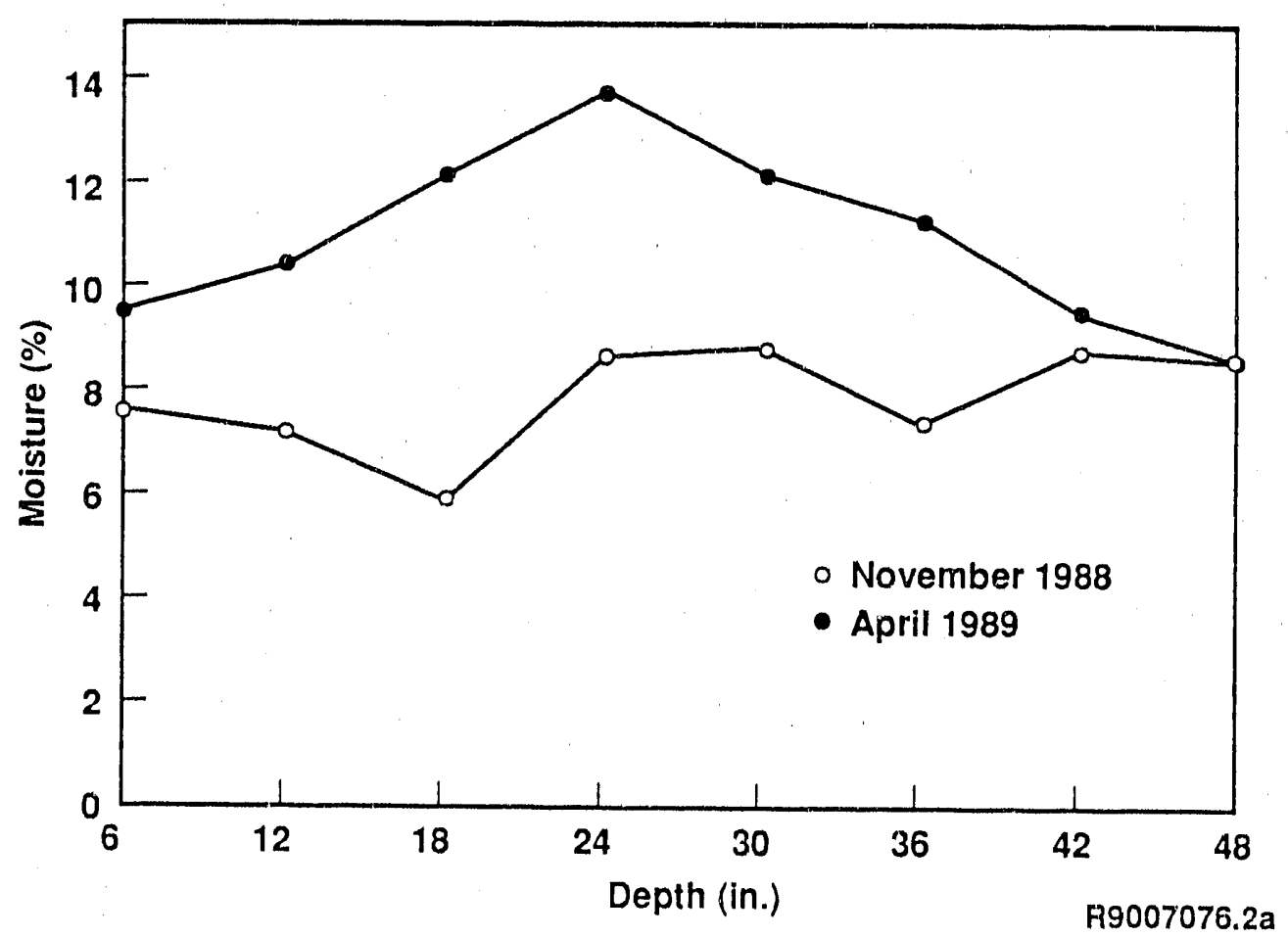

Figure 8. Soil Moisture Profile of the Pocket Gopher. 


\subsection{REFERENCES}

Adams, M. R. and N. R. Wing, 1986, Protective Barrier and Warning Marker System Development Plan, RHO-RE-PL-35P, Rockwell Hanford Operations, Richland, Washington.

Last, G. V., M. A. Glennon, M. A. Young, and G. W. Gee, 1987, Protective Barrier Materials Analysis: Fine Soil Site Characterization, PNL-6314, Pacific Northwest Laboratory, Richland, Washington. 
WHC-EP-0299

APPENDIX A

SOIL RESISTANCE DATA

$$
A-i / A i
$$


Table A-1. Resistance Readings (Ohms) Taken May 12, 1988.

\begin{tabular}{lllllll}
\hline $\begin{array}{l}\text { Depth } \\
\text { (in.) }\end{array}$ & $\begin{array}{l}\text { Box } \\
\text { in }\end{array}$ & $\begin{array}{l}\text { Log } \\
\text { 2A }\end{array}$ & $\begin{array}{l}\text { Box } \\
2 B\end{array}$ & $\begin{array}{l}\text { Log } \\
2 B\end{array}$ & $\begin{array}{l}\text { Bux } \\
2 C\end{array}$ & $\begin{array}{l}\text { Log } \\
2 C\end{array}$ \\
\hline & & & & & & \\
2.0 & 595000 & 5.8 & 2400000 & 6.4 & 22800 & 4.4 \\
4.0 & 86900 & 4.9 & 2360000 & 6.4 & 25500 & 4.4 \\
6.0 & 27900 & 4.4 & 19200 & 4.3 & 13800 & 4.1 \\
8.0 & 16000 & 4.2 & 1520 & 3.2 & 13200 & 4.1 \\
10.0 & 10600 & 4.0 & 7070 & 3.8 & 10700 & 4.0 \\
12.0 & 8420 & 3.9 & 6570 & 3.8 & 8090 & 3.9 \\
14.0 & 7970 & 3.9 & 6150 & 3.8 & 7360 & 3.9 \\
16.0 & 8160 & 3.9 & 5900 & 3.8 & 6520 & 3.8 \\
18.0 & 6520 & 3.8 & 6200 & 3.8 & 5610 & 3.7 \\
20.0 & 5010 & 3.7 & 5710 & 3.8 & 5520 & 3.7 \\
22.0 & 4880 & 3.7 & 4630 & 3.7 & 5750 & 3.8 \\
24.0 & 5380 & 3.7 & 5380 & 3.7 & 5470 & 3.7 \\
26.0 & 4450 & 3.6 & 5560 & 3.7 & 4690 & 3.7 \\
28.0 & 4390 & 3.6 & 5380 & 3.7 & 11200 & 4.0 \\
30.0 & 5280 & 3.7 & 6570 & 3.8 & 12300 & 4.1 \\
32.0 & 6300 & 3.8 & 6620 & 3.8 & 3240 & 3.5 \\
34.0 & 7780 & 3.9 & 6200 & 3.8 & 7240 & 3.9 \\
36.0 & 7900 & 3.9 & 6520 & 3.8 & 6250 & 3.8 \\
38.0 & 7420 & 3.9 & 6950 & 3.8 & 5610 & 3.7 \\
40.0 & 6730 & 3.8 & 6620 & 3.8 & 4690 & 3.7 \\
42.0 & 5560 & 3.7 & 5330 & 3.7 & 4170 & 3.6 \\
44.0 & 5470 & 3.7 & 5710 & 3.8 & 4020 & 3.6
\end{tabular}


Table A-2. Resistance Readings (Ohms) Taken May 19, 1988.

\begin{tabular}{|c|c|c|c|c|c|c|}
\hline $\begin{array}{l}\text { Depth } \\
\text { (in.) }\end{array}$ & $\begin{array}{l}\text { Box } \\
1 A\end{array}$ & $\begin{array}{l}\log \\
1 A\end{array}$ & $\begin{array}{l}\text { Box } \\
1 B\end{array}$ & $\begin{array}{l}\log \\
1 B\end{array}$ & $\begin{array}{l}\text { Box } \\
\text { IC }\end{array}$ & $\begin{array}{l}\log \\
1 C\end{array}$ \\
\hline $\begin{array}{l}2.0 \\
4.0 \\
6.0 \\
8.0 \\
10.0 \\
12.0 \\
14.0 \\
16.0 \\
18.0 \\
20.0 \\
22.0 \\
24.0 \\
26.0 \\
28.0 \\
30.0 \\
32.0 \\
34.0 \\
36.0 \\
38.0 \\
40.0 \\
42.0 \\
44.0\end{array}$ & $\begin{array}{r}809000 \\
\\
6950 \\
15600 \\
17100 \\
12400 \\
10200 \\
6250 \\
13100 \\
13400 \\
6620 \\
9250 \\
9110 \\
6200 \\
5190 \\
34500 \\
36100 \\
10600 \\
11200 \\
7970 \\
718000\end{array}$ & $\begin{array}{l}5.9 \\
\text { ERR } \\
\text { ERR } \\
3.8 \\
4.2 \\
4.2 \\
4.1 \\
4.0 \\
3.8 \\
4.1 \\
4.1 \\
3.8 \\
4.0 \\
4.0 \\
3.8 \\
3.7 \\
4.5 \\
4.6 \\
4.0 \\
4.0 \\
3.9 \\
5.9\end{array}$ & $\begin{array}{r}1280000 \\
1300000 \\
417000 \\
423000 \\
1890000 \\
2180000 \\
18890 \\
8690 \\
1090 \\
69500 \\
65700 \\
\\
\\
6680 \\
8350 \\
11100 \\
9110 \\
889000 \\
1120000 \\
65200 \\
11000 \\
7720\end{array}$ & $\begin{array}{l}6.1 \\
6.1 \\
5.6 \\
5.6 \\
6.3 \\
6.3 \\
4.3 \\
3.9 \\
3.0 \\
4.8 \\
4.8 \\
\text { ERR } \\
\text { ERR } \\
3.8 \\
3.9 \\
4.0 \\
4.0 \\
5.9 \\
6.0 \\
4.8 \\
4.0 \\
3.9\end{array}$ & $\begin{array}{r}112000 \\
12100 \\
20100 \\
82200 \\
67300 \\
6840 \\
6900 \\
9690 \\
10100 \\
8030 \\
8420 \\
8550 \\
7600 \\
6680 \\
191000 \\
192000 \\
1090000 \\
1510000\end{array}$ & $\begin{array}{l}\text { ERR } \\
\text { ERR } \\
\text { ERR } \\
5.0 \\
4.1 \\
4.3 \\
4.9 \\
4.8 \\
3.8 \\
3.8 \\
4.0 \\
4.0 \\
3.9 \\
3.9 \\
3.9 \\
3.9 \\
3.8 \\
5.3 \\
5.3 \\
6.0 \\
6.2 \\
\text { ERR }\end{array}$ \\
\hline
\end{tabular}


Table A-3. Resistance Readings (Ohms) Taken July 28, 1988 (Prerainulator).

\begin{tabular}{|c|c|c|c|c|c|c|}
\hline $\begin{array}{l}\text { Depth } \\
(\text { in. })\end{array}$ & $\begin{array}{l}\text { Box } \\
1 A\end{array}$ & $\begin{array}{l}\log \\
1 A\end{array}$ & $\begin{array}{l}\text { BoX } \\
1 B\end{array}$ & $\begin{array}{l}\log \\
1 B\end{array}$ & $\begin{array}{l}\text { Box } \\
1 C\end{array}$ & $\begin{array}{l}\log \\
1 C\end{array}$ \\
\hline & & & & & & - \\
\hline $\begin{array}{l}2.0 \\
4.0 \\
6.0 \\
8.0 \\
10.0 \\
12.0 \\
14.0 \\
16.0 \\
18.0 \\
20.0 \\
22.0 \\
24.0 \\
26.0 \\
28.0 \\
30.0 \\
32.0 \\
34.0 \\
36.0 \\
38.0 \\
40.0 \\
42.0 \\
44.0\end{array}$ & $\begin{array}{r}288000 \\
313000 \\
256000 \\
12800 \\
23200 \\
23200 \\
18400 \\
15100 \\
7840 \\
39200 \\
38800 \\
9540 \\
189000 \\
186000 \\
10200 \\
8220 \\
222000 \\
216000 \\
13400 \\
12500 \\
8610 \\
34500\end{array}$ & $\begin{array}{l}5.5 \\
5.5 \\
5.4 \\
4.1 \\
4.4 \\
4.4 \\
4.3 \\
4.2 \\
3.9 \\
4.6 \\
4.6 \\
4.0 \\
5.3 \\
5.3 \\
4.0 \\
3.9 \\
5.3 \\
5.3 \\
4.1 \\
4.1 \\
3.9 \\
4.5\end{array}$ & $\begin{array}{r}109000 \\
155000 \\
186000 \\
366000 \\
370000 \\
114000 \\
25100 \\
46300 \\
134000 \\
104000 \\
4500000 \\
4200000 \\
16800 \\
168000 \\
203000 \\
34900 \\
143000 \\
469000 \\
316000 \\
15500 \\
11400\end{array}$ & $\begin{array}{l}\text { ERR } \\
5.0 \\
5.2 \\
5.3 \\
5.6 \\
5.6 \\
5.1 \\
4.4 \\
4.7 \\
5.1 \\
5.0 \\
6.7 \\
6.6 \\
4.2 \\
5.2 \\
5.3 \\
4.5 \\
5.2 \\
5.7 \\
5.5 \\
4.2 \\
4.1\end{array}$ & $\begin{array}{r}1440000 \\
191000 \\
149000 \\
16200 \\
16400 \\
8760 \\
21600 \\
27100 \\
18100 \\
18600 \\
1550000 \\
1510000 \\
11700 \\
11300 \\
17600 \\
36100 \\
24600 \\
27400 \\
42300 \\
91800 \\
947000 \\
1240000\end{array}$ & $\begin{array}{l}6.2 \\
5.3 \\
5.2 \\
4.2 \\
4.2 \\
3.9 \\
4.3 \\
4.4 \\
4.3 \\
4.3 \\
6.2 \\
6.2 \\
4.1 \\
4.1 \\
4.2 \\
4.6 \\
4.4 \\
4.4 \\
4.6 \\
5.0 \\
6.0 \\
6.1\end{array}$ \\
\hline
\end{tabular}


Table A-4. Reststance Readings (Ohms) Taken July 28, 1988

(Prerainulator).

\begin{tabular}{|c|c|c|c|c|c|c|}
\hline $\begin{array}{l}\text { Depth } \\
(\text { in.) }\end{array}$ & $\begin{array}{l}\text { Box } \\
2 A\end{array}$ & $\begin{array}{l}\log \\
2 A\end{array}$ & $\begin{array}{l}\text { Box } \\
2 B\end{array}$ & $\begin{array}{l}\log \\
2 B\end{array}$ & $\begin{array}{l}\text { Box } \\
2 C\end{array}$ & $\begin{array}{l}\log \\
2 C\end{array}$ \\
\hline $\begin{array}{l}2.0 \\
4.0 \\
6.0 \\
8.0 \\
10.0 \\
12.0 \\
14.0 \\
16.0 \\
18.0 \\
20.0 \\
22.0 \\
24.0 \\
26.0 \\
28.0 \\
30.0 \\
32.0 \\
34.0 \\
36.0 \\
38.0 \\
40.0 \\
42.0 \\
44.0\end{array}$ & $\begin{array}{r}3210000 \\
41200 \\
20300 \\
13800 \\
10800 \\
9850 \\
9390 \\
10000 \\
8420 \\
6680 \\
7240 \\
7420 \\
6840 \\
8160 \\
9180 \\
9540 \\
12900 \\
14700 \\
14900 \\
12600 \\
10200 \\
10900\end{array}$ & $\begin{array}{l}6.5 \\
4.6 \\
4.3 \\
4.1 \\
4.0 \\
4.0 \\
4.0 \\
4.0 \\
3.9 \\
3.8 \\
3.9 \\
3.9 \\
3.8 \\
3.9 \\
4.0 \\
4.0 \\
4.1 \\
4.2 \\
4.2 \\
4.1 \\
4.0 \\
4.0\end{array}$ & $\begin{array}{r}220000 \\
186000 \\
86900 \\
97700 \\
70100 \\
32000 \\
31600 \\
26600 \\
30000 \\
24100 \\
16200 \\
22000 \\
22200 \\
19600 \\
20700 \\
17500 \\
18900 \\
31000 \\
33800 \\
18900 \\
9030 \\
8160\end{array}$ & $\begin{array}{l}5.3 \\
5.3 \\
4.9 \\
5.0 \\
4.8 \\
4.5 \\
4.5 \\
4.4 \\
4.5 \\
4.4 \\
4.2 \\
4.3 \\
4.3 \\
4.3 \\
4.3 \\
4.2 \\
4.3 \\
4.5 \\
4.5 \\
4.3 \\
4.0 \\
3.9\end{array}$ & $\begin{array}{r}463000 \\
165000 \\
47500 \\
25800 \\
25300 \\
17100 \\
12600 \\
9920 \\
8420 \\
8090 \\
8550 \\
7840 \\
6790 \\
14700 \\
16400 \\
9320 \\
8760 \\
8290 \\
7900 \\
6900 \\
6200 \\
5850\end{array}$ & $\begin{array}{l}5.7 \\
5.2 \\
4.7 \\
4.4 \\
4.4 \\
4.2 \\
4.1 \\
4.0 \\
3.9 \\
3.9 \\
3.9 \\
3.9 \\
3.8 \\
4.2 \\
4.2 \\
4.0 \\
3.9 \\
3.9 \\
3.9 \\
3.8 \\
3.8 \\
3.8\end{array}$ \\
\hline
\end{tabular}


Table A-5. Soll Reststance Readings (Ohms) Taken July 28, 1988 (2-Hour Postrainulator).

\begin{tabular}{|c|c|c|c|c|c|c|}
\hline $\begin{array}{l}\text { Depth } \\
(\text { in.) }\end{array}$ & $\begin{array}{l}\text { Box } \\
1 A\end{array}$ & $\begin{array}{l}\log \\
1 A\end{array}$ & $\begin{array}{l}B 0 x \\
1 B\end{array}$ & $\begin{array}{l}\log \\
18\end{array}$ & $\begin{array}{l}\text { Box } \\
1 C\end{array}$ & $\begin{array}{l}\log \\
1 C\end{array}$ \\
\hline $\begin{array}{l}2.0 \\
4.0 \\
6.0 \\
8.0 \\
10.0 \\
12.0 \\
14.0 \\
16.0 \\
18.0 \\
20.0 \\
22.0 \\
24.0 \\
26.0 \\
28.0 \\
30.0 \\
32.0 \\
34.0 \\
36.0 \\
38.0 \\
40.0 \\
42.0 \\
44.0\end{array}$ & $\begin{array}{r}20100 \\
220000 \\
216000 \\
13700 \\
25100 \\
25300 \\
20100 \\
16400 \\
8550 \\
46300 \\
45700 \\
10300 \\
214000 \\
212000 \\
10900 \\
8610 \\
248000 \\
244000 \\
13700 \\
12900 \\
9390 \\
44500\end{array}$ & $\begin{array}{l}4.3 \\
5.3 \\
5.3 \\
4.1 \\
4.4 \\
4.4 \\
4.3 \\
4.2 \\
3.9 \\
4.7 \\
4.7 \\
4.0 \\
5.3 \\
5.3 \\
4.0 \\
3.9 \\
5.4 \\
5.4 \\
4.1 \\
4.1 \\
4.0 \\
4.6\end{array}$ & $\begin{array}{r}69500 \\
34900 \\
134000 \\
205000 \\
397000 \\
402000 \\
122000 \\
27100 \\
73600 \\
145000 \\
114000 \\
4680000 \\
4500000 \\
17400 \\
198000 \\
235000 \\
37000 \\
157000 \\
552000 \\
341000 \\
15600 \\
13500\end{array}$ & $\begin{array}{l}4.8 \\
4.5 \\
5.1 \\
5.3 \\
5.6 \\
5.6 \\
5.1 \\
4.4 \\
4.9 \\
5.2 \\
5.1 \\
6.7 \\
6.7 \\
4.2 \\
5.3 \\
5.4 \\
4.6 \\
5.2 \\
5.7 \\
5.5 \\
4.2 \\
4.1\end{array}$ & $\begin{array}{r}226000 \\
143000 \\
96900 \\
45700 \\
29400 \\
26100 \\
36100 \\
28200 \\
11700 \\
12201 \\
1030000 \\
992000 \\
19200 \\
18800 \\
28500 \\
22200 \\
8820 \\
16900 \\
16900 \\
172000 \\
212000 \\
2090000\end{array}$ & $\begin{array}{l}5.1 \\
5.2 \\
5.0 \\
4.7 \\
4.5 \\
4.4 \\
4.6 \\
4.5 \\
4.1 \\
4.1 \\
6.0 \\
6.0 \\
4.3 \\
4.3 \\
4.5 \\
4.3 \\
3.9 \\
4.2 \\
4.2 \\
5.2 \\
5.3 \\
6.3\end{array}$ \\
\hline
\end{tabular}


Table A-6. Soll Resistance Readings (Ohms) Taken July 30, 1988 (48-Hour Postrainulator).

\begin{tabular}{|c|c|c|c|c|c|c|}
\hline $\begin{array}{l}\text { Depth } \\
(\ln .)\end{array}$ & $\begin{array}{l}B \cap x \\
1 A\end{array}$ & $\begin{array}{l}\log \\
1 A\end{array}$ & $\begin{array}{l}\text { Box } \\
1 B\end{array}$ & $\begin{array}{l}\log \\
1 B\end{array}$ & $\begin{array}{l}\text { Box } \\
1 C\end{array}$ & $\begin{array}{l}\log \\
1 C\end{array}$ \\
\hline $\begin{array}{l}2.0 \\
4.0 \\
6.0 \\
8.0 \\
10.0 \\
12.0 \\
14.0 \\
16.0 \\
18.0 \\
20.0 \\
22.0 \\
24.0 \\
26.0 \\
28.0 \\
30.0 \\
32.0 \\
34.0 \\
36.0 \\
38.0 \\
40.0 \\
42.0 \\
44.0\end{array}$ & $\begin{array}{r}96200 \\
282000 \\
248000 \\
14400 \\
26800 \\
27400 \\
23200 \\
19100 \\
9620 \\
74200 \\
74700 \\
11300 \\
226000 \\
220000 \\
11800 \\
9390 \\
279000 \\
274000 \\
14400 \\
13300 \\
9770 \\
42300\end{array}$ & $\begin{array}{l}5.0 \\
5.5 \\
5.4 \\
4.2 \\
4.4 \\
4.4 \\
4.4 \\
4.3 \\
4.0 \\
4.9 \\
4.9 \\
4.1 \\
5.4 \\
5.3 \\
4.1 \\
4.0 \\
5.4 \\
5.4 \\
4.2 \\
4.1 \\
4.0 \\
4.6\end{array}$ & $\begin{array}{r}108000 \\
102000 \\
155000 \\
186000 \\
374000 \\
383000 \\
120000 \\
30300 \\
81600 \\
161000 \\
128000 \\
4530000 \\
4240000 \\
19200 \\
214000 \\
255000 \\
41200 \\
188000 \\
657000 \\
374000 \\
18400 \\
15100\end{array}$ & $\begin{array}{l}5.0 \\
5.0 \\
5.2 \\
5.3 \\
5.6 \\
5.6 \\
5.1 \\
4.5 \\
4.9 \\
5.2 \\
5.1 \\
6.7 \\
6.6 \\
4.3 \\
5.3 \\
5.4 \\
4.6 \\
5.3 \\
5.8 \\
5.6 \\
4.3 \\
4.2\end{array}$ & $\begin{array}{r}428000 \\
172000 \\
88900 \\
44500 \\
30600 \\
28200 \\
37400 \\
29100 \\
12400 \\
12900 \\
772000 \\
790000 \\
21000 \\
19800 \\
31000 \\
24600 \\
9710 \\
18100 \\
18100 \\
198000 \\
241000 \\
3130000\end{array}$ & $\begin{array}{l}5.6 \\
5.2 \\
4.9 \\
4.6 \\
4.5 \\
4.5 \\
4.6 \\
4.5 \\
4.1 \\
4.1 \\
5.9 \\
5.9 \\
4.3 \\
4.3 \\
4.5 \\
4.4 \\
4.0 \\
4.3 \\
4.3 \\
5.3 \\
5.4 \\
6.5\end{array}$ \\
\hline
\end{tabular}


Table A-7. Resistance Readings (Ohms) Taken August 2, 1988 (96-Hour Postratnulator).

\begin{tabular}{|c|c|c|c|c|c|c|}
\hline $\begin{array}{l}\text { Depth } \\
(1 n .)\end{array}$ & $\begin{array}{l}\text { Box } \\
\text { IA }\end{array}$ & $\begin{array}{l}\log \\
1 A\end{array}$ & $\begin{array}{l}B a x \\
1 B\end{array}$ & $\begin{array}{l}\log \\
1 B\end{array}$ & $\begin{array}{l}\text { Box } \\
1 C\end{array}$ & $\begin{array}{l}\log \\
1 C\end{array}$ \\
\hline $\begin{array}{l}2.0 \\
4.0 \\
6.0 \\
8.0 \\
10.0 \\
12.0 \\
14.0 \\
16.0 \\
18.0 \\
20.0 \\
22.0 \\
24.0 \\
26.0 \\
28.0 \\
30.0 \\
32.0 \\
34.0 \\
36.0 \\
38.0 \\
40.0 \\
42.0 \\
44.0\end{array}$ & $\begin{array}{r}106000 \\
428000 \\
379000 \\
16200 \\
29400 \\
26700 \\
25500 \\
20700 \\
10400 \\
87600 \\
85500 \\
12100 \\
268000 \\
266000 \\
12600 \\
10100 \\
267000 \\
291000 \\
15500 \\
13900 \\
9690 \\
57100\end{array}$ & $\begin{array}{l}5.0 \\
5.6 \\
5.6 \\
4.2 \\
4.5 \\
4.4 \\
4.4 \\
4.3 \\
4.0 \\
4.9 \\
4.9 \\
4.1 \\
5.4 \\
5.4 \\
4.1 \\
4.0 \\
5.4 \\
5.5 \\
4.2 \\
4.1 \\
4.0 \\
4.8\end{array}$ & $\begin{array}{r}153000 \\
130000 \\
207000 \\
239000 \\
433000 \\
445000 \\
137000 \\
32700 \\
87600 \\
175000 \\
139000 \\
3440000 \\
3470000 \\
20700 \\
239000 \\
282000 \\
44500 \\
205000 \\
718000 \\
397000 \\
20800 \\
14400\end{array}$ & $\begin{array}{l}5.2 \\
5.1 \\
5.3 \\
5.4 \\
5.6 \\
5.6 \\
5.1 \\
4.5 \\
4.9 \\
5.2 \\
5.1 \\
6.5 \\
6.5 \\
4.3 \\
5.4 \\
5.5 \\
4.6 \\
5.3 \\
5.9 \\
5.6 \\
4.3 \\
4.2\end{array}$ & $\begin{array}{r}392000 \\
276000 \\
119000 \\
87600 \\
34100 \\
30000 \\
39200 \\
30000 \\
13000 \\
13500 \\
152000 \\
889000 \\
23000 \\
20500 \\
33800 \\
27100 \\
10200 \\
18900 \\
19200 \\
220000 \\
271000 \\
194000\end{array}$ & $\begin{array}{l}5.6 \\
5.4 \\
5.1 \\
4.9 \\
4.5 \\
4.5 \\
4.6 \\
4.5 \\
4.1 \\
4.1 \\
5.2 \\
5.9 \\
4.4 \\
4.3 \\
4.5 \\
4.4 \\
4.0 \\
4.3 \\
4.3 \\
5.3 \\
5.4 \\
5.3\end{array}$ \\
\hline
\end{tabular}


Table A-8. Resistance Readings (Ohms) Taken August 29, 1988 (Preratnulator).

\begin{tabular}{lrrrrrr}
\hline $\begin{array}{l}\text { Depth } \\
\text { (1n.) }\end{array}$ & Box & Log & Box & Log & Box & Log \\
& $1 A$ & $1 A$ & $1 B$ & $1 B$ & $1 C$ & $1 C$ \\
\hline 2.0 & 291000 & 5.5 & 3210000 & 6.5 & 3810000 & 6.6 \\
4.0 & 615000 & 5.8 & 180000 & 5.3 & 1460000 & 6.2 \\
6.0 & 495000 & 5.7 & 266000 & 5.14 & 138000 & 5.1 \\
8.0 & 18400 & 4.3 & 258000 & 5.4 & 104000 & 5.0 \\
10.0 & 42800 & 4.6 & 463000 & 5.7 & 40700 & 4.6 \\
12.0 & 72400 & 4.9 & 547000 & 5.7 & 36600 & 4.6 \\
14.0 & 33400 & 4.5 & 153000 & 5.2 & 42300 & 4.6 \\
16.0 & 27400 & 4.4 & 37000 & 4.6 & 33400 & 4.5 \\
18.0 & 12000 & 4.1 & 92500 & 5.0 & 15700 & 4.2 \\
20.0 & 95400 & 5.0 & 133000 & 5.3 & 17700 & 4.2 \\
22.0 & 96200 & 5.0 & 151000 & 5.2 & 4000000 & 6.6 \\
24.0 & 13900 & 4.1 & 6360000 & 6.8 & 4120000 & 6.6 \\
26.0 & 294000 & 5.5 & 6560000 & 6.8 & 32300 & 4.5 \\
28.0 & 291000 & 5.5 & 24400 & 4.4 & 29400 & 4.5 \\
30.0 & 14900 & 4.2 & 239000 & 5.4 & 43900 & 4.6 \\
32.0 & 12200 & 4.1 & 279000 & 5.4 & 33000 & 4.5 \\
34.0 & 276000 & 5.4 & 74200 & 4.9 & 12200 & 4.1 \\
36.0 & 276000 & 5.4 & 208000 & 5.3 & 23000 & 4.4 \\
38.0 & 19900 & 4.3 & 797000 & 5.9 & 22600 & 4.4 \\
40.0 & 18300 & 4.3 & 451000 & 5.7 & 244000 & 5.4 \\
42.0 & 12300 & 4.1 & 283400 & 4.5 & 310000 & 5.5 \\
44.0 & 43300 & 4.6 & 18400 & 4.3 & 8280000 & 6.9 \\
& & & & & &
\end{tabular}


Table A-9. Resistance Readings (Ohms) Taken August 29, 1988 (Prerainulator).

\begin{tabular}{lrlllll}
\hline $\begin{array}{l}\text { Depth } \\
\text { (1n.) }\end{array}$ & $\begin{array}{l}\text { Box } \\
\text { 2A }\end{array}$ & $\begin{array}{l}\text { Log } \\
2 A\end{array}$ & $\begin{array}{l}\text { Box } \\
2 B\end{array}$ & $\begin{array}{l}\text { Log } \\
2 B\end{array}$ & $\begin{array}{l}\text { Box } \\
2 C\end{array}$ & $\begin{array}{l}\text { Log } \\
2 C\end{array}$ \\
\hline 2.0 & 6720000 & 6.8 & 334000 & 5.5 & 695000 & 5.8 \\
4.0 & 79700 & 4.9 & 300000 & 5.5 & 153000 & 5.2 \\
6.0 & 27900 & 4.4 & 117000 & 5.1 & 87600 & 4.9 \\
8.0 & 18400 & 4.3 & 136000 & 5.1 & 80300 & 4.9 \\
10.0 & 13900 & 4.1 & 98500 & 5.0 & 26700 & 4.4 \\
12.0 & 12600 & 4.1 & 72400 & 4.9 & 23200 & 4.4 \\
14.0 & 12200 & 4.1 & 81600 & 4.9 & 16100 & 4.2 \\
16.0 & 12700 & 4.1 & 41200 & 4.6 & 12900 & 4.1 \\
18.0 & 10700 & 4.0 & 44500 & 4.6 & 11200 & 4.0 \\
20.0 & 8820 & 3.9 & 33800 & 4.5 & 10900 & 4.0 \\
22.0 & 9690 & 4.0 & 23700 & 4.4 & 11200 & 4.0 \\
24.0 & 10000 & 4.0 & 32000 & 4.5 & 10100 & 4.0 \\
26.0 & 9390 & 4.0 & 32300 & 4.5 & 8610 & 3.9 \\
28.0 & 10800 & 4.0 & 27900 & 4.4 & 19600 & 4.3 \\
30.0 & 11500 & 4.1 & 29400 & 4.5 & 21800 & 4.3 \\
32.0 & 12000 & 4.1 & 25300 & 4.4 & 12600 & 4.1 \\
34.0 & 16400 & 4.2 & 30000 & 4.5 & 11500 & 4.1 \\
36.0 & 18600 & 4.3 & 66200 & 4.8 & 10400 & 4.0 \\
38.0 & 18600 & 4.3 & 74200 & 4.9 & 10000 & 4.0 \\
40.0 & 15100 & 4.2 & 27100 & 4.4 & 8820 & 3.9 \\
42.0 & 11800 & 4.1 & 13000 & 4.1 & 8030 & 3.9 \\
44.0 & 10600 & 4.0 & 12100 & 4.1 & 6950 & 3.8 \\
& & & & & &
\end{tabular}


Table A-10. Resistance Readings (Ohms) Taken August 30, 1988 (2-Hour Postrainulator).

\begin{tabular}{|c|c|c|c|c|c|c|}
\hline $\begin{array}{l}\text { Depth } \\
(\text { in.) }\end{array}$ & $\begin{array}{l}\text { Box } \\
\text { :A }\end{array}$ & $\begin{array}{l}\log \\
\text { IA }\end{array}$ & $\begin{array}{l}\text { Box } \\
1 B\end{array}$ & $\begin{array}{l}\log \\
1 B\end{array}$ & $\begin{array}{l}\text { Box } \\
\text { IC }\end{array}$ & $\begin{array}{l}\log \\
1 C\end{array}$ \\
\hline $\begin{array}{l}2.0 \\
4.0 \\
6.0 \\
8.0 \\
10.0 \\
12.0 \\
14.0 \\
16.0 \\
18.0 \\
20.0 \\
22.0 \\
24.0 \\
26.0 \\
28.0 \\
30.0 \\
32.0 \\
34.0 \\
36.0 \\
38.0 \\
40.0 \\
42.0 \\
44.0\end{array}$ & $\begin{array}{r}32300 \\
493000 \\
451000 \\
19900 \\
47500 \\
79700 \\
37400 \\
30600 \\
12500 \\
100000 \\
99900 \\
14300 \\
320000 \\
316000 \\
15200 \\
12300 \\
310000 \\
306000 \\
20700 \\
18900 \\
12600 \\
67300\end{array}$ & $\begin{array}{l}4.5 \\
5.7 \\
5.7 \\
4.3 \\
4.7 \\
4.9 \\
4.6 \\
4.5 \\
4.1 \\
5.0 \\
5.0 \\
4.2 \\
5.5 \\
5.5 \\
4.2 \\
4.1 \\
5.5 \\
5.5 \\
4.3 \\
4.3 \\
4.1 \\
4.8\end{array}$ & $\begin{array}{r}172000 \\
133000 \\
267000 \\
294000 \\
556000 \\
600000 \\
161000 \\
39200 \\
97700 \\
196000 \\
159000 \\
6400000 \\
6610000 \\
25500 \\
266000 \\
306000 \\
78400 \\
224000 \\
816000 \\
463000 \\
30000 \\
19200\end{array}$ & $\begin{array}{l}5.2 \\
5.1 \\
5.4 \\
5.5 \\
5.7 \\
5.8 \\
5.2 \\
4.6 \\
5.0 \\
5.3 \\
5.2 \\
6.8 \\
6.8 \\
4.4 \\
5.4 \\
5.5 \\
4.9 \\
5.4 \\
5.9 \\
5.7 \\
4.5 \\
4.3\end{array}$ & $\begin{array}{r}947000 \\
954000 \\
161000 \\
121000 \\
44500 \\
38300 \\
43900 \\
34500 \\
16400 \\
18400 \\
3640000 \\
3700000 \\
34500 \\
31300 \\
46300 \\
34500 \\
12300 \\
23500 \\
23500 \\
274000 \\
341000 \\
4240000\end{array}$ & $\begin{array}{l}6.0 \\
6.0 \\
5.2 \\
5.1 \\
4.6 \\
4.6 \\
4.6 \\
4.5 \\
4.2 \\
4.3 \\
6.6 \\
6.6 \\
4.5 \\
4.5 \\
4.7 \\
4.5 \\
4.1 \\
4.4 \\
4.4 \\
5.4 \\
5.5 \\
6.6\end{array}$ \\
\hline
\end{tabular}


Table A-11. Resistance Readings (Ohms) Taken September 1, 1988 (48-Hour Postrainulator).

\begin{tabular}{|c|c|c|c|c|c|c|}
\hline $\begin{array}{l}\text { Depth } \\
\text { (in.) }\end{array}$ & $\begin{array}{l}\text { Box } \\
1 A\end{array}$ & $\begin{array}{l}\log \\
1 A\end{array}$ & $\begin{array}{l}\text { Box } \\
1 B\end{array}$ & $\begin{array}{l}\log \\
1 B\end{array}$ & $\begin{array}{l}\text { Box } \\
1 C\end{array}$ & $\begin{array}{l}\log \\
1 C\end{array}$ \\
\hline $\begin{array}{l}2.0 \\
4.0 \\
6.0 \\
8.0 \\
10.0 \\
12.0 \\
14.0 \\
16.0 \\
18.0 \\
20.0 \\
22.0 \\
24.0 \\
26.0 \\
28.0 \\
30.0 \\
32.0 \\
34.0 \\
36.0 \\
38.0 \\
40.0 \\
42.0 \\
44.0\end{array}$ & $\begin{array}{r}150000 \\
690000 \\
590000 \\
21600 \\
51000 \\
44500 \\
34500 \\
34900 \\
13400 \\
123000 \\
122000 \\
15300 \\
338000 \\
334000 \\
16100 \\
13000 \\
349000 \\
345000 \\
22400 \\
20300 \\
12500 \\
78400\end{array}$ & $\begin{array}{l}5.2 \\
5.8 \\
5.8 \\
4.3 \\
4.7 \\
4.6 \\
4.5 \\
4.5 \\
4.1 \\
5.1 \\
5.1 \\
4.2 \\
5.5 \\
5.5 \\
4.2 \\
4.1 \\
5.5 \\
5.5 \\
4.4 \\
4.3 \\
4.1 \\
4.9\end{array}$ & $\begin{array}{r}357000 \\
226000 \\
320000 \\
285000 \\
585000 \\
630000 \\
162000 \\
43300 \\
106000 \\
214000 \\
177000 \\
8720000 \\
9040000 \\
28800 \\
310000 \\
357000 \\
86900 \\
253000 \\
889000 \\
489000 \\
34500 \\
21600\end{array}$ & $\begin{array}{l}5.6 \\
5.4 \\
5.5 \\
5.5 \\
5.8 \\
5.8 \\
5.2 \\
4.6 \\
5.0 \\
5.3 \\
5.2 \\
6.9 \\
7.0 \\
4.5 \\
5.5 \\
5.6 \\
4.9 \\
5.4 \\
5.9 \\
5.7 \\
4.5 \\
4.3\end{array}$ & $\begin{array}{r}1390000 \\
1180000 \\
171000 \\
128000 \\
83500 \\
42800 \\
74700 \\
37400 \\
18000 \\
20500 \\
2800000 \\
2820000 \\
40200 \\
36600 \\
86100 \\
39200 \\
13000 \\
25800 \\
25500 \\
323000 \\
407000 \\
5040000\end{array}$ & $\begin{array}{l}6.1 \\
6.1 \\
5.2 \\
5.1 \\
4.9 \\
4.6 \\
4.9 \\
4.6 \\
4.3 \\
4.3 \\
6.4 \\
6.5 \\
4.6 \\
4.6 \\
4.9 \\
4.6 \\
4.1 \\
4.4 \\
4.4 \\
5.5 \\
5.6 \\
6.7\end{array}$ \\
\hline
\end{tabular}


Table A-12. Resistance Readings (Ohms) Taken September 10, 1988.

\begin{tabular}{|c|c|c|c|c|c|c|}
\hline $\begin{array}{l}\text { Depth } \\
(\text { in. })\end{array}$ & $\begin{array}{l}\text { Box } \\
1 A\end{array}$ & $\begin{array}{l}\log \\
1 A\end{array}$ & $\begin{array}{l}\text { Box } \\
\text { IB }\end{array}$ & $\begin{array}{l}\log \\
1 B\end{array}$ & $\begin{array}{l}\text { Box } \\
\text { IC }\end{array}$ & $\begin{array}{l}\log \\
1 C\end{array}$ \\
\hline $\begin{array}{l}2.0 \\
4.0 \\
6.0 \\
8.0 \\
10.0 \\
12.0 \\
14.0 \\
16.0 \\
18.0 \\
20.0 \\
22.0 \\
24.0 \\
26.0 \\
28.0 \\
30.0 \\
32.0 \\
34.0 \\
36.0 \\
38.0 \\
40.0 \\
42.0 \\
44.0\end{array}$ & $\begin{array}{r}157000 \\
695000 \\
600000 \\
21000 \\
51000 \\
47500 \\
36600 \\
33800 \\
13200 \\
112000 \\
112000 \\
15200 \\
334000 \\
330000 \\
16100 \\
12900 \\
285000 \\
285000 \\
22400 \\
20800 \\
13600 \\
78400\end{array}$ & $\begin{array}{l}5.2 \\
5.8 \\
5.8 \\
4.3 \\
4.7 \\
4.7 \\
4.6 \\
4.5 \\
4.1 \\
5.0 \\
5.0 \\
4.2 \\
5.5 \\
5.5 \\
4.2 \\
4.1 \\
5.5 \\
5.5 \\
4.4 \\
4.3 \\
4.1 \\
4.9\end{array}$ & $\begin{array}{r}707000 \\
210000 \\
327000 \\
282000 \\
566000 \\
610000 \\
167000 \\
41700 \\
102000 \\
205000 \\
169000 \\
6960000 \\
7200000 \\
29400 \\
268000 \\
316000 \\
85500 \\
230000 \\
876000 \\
495000 \\
36100 \\
21800\end{array}$ & $\begin{array}{l}5.8 \\
5.3 \\
5.5 \\
5.5 \\
5.8 \\
5.8 \\
5.2 \\
4.6 \\
5.0 \\
5.3 \\
5.2 \\
6.8 \\
6.9 \\
4.5 \\
5.4 \\
5.5 \\
4.9 \\
5.4 \\
5.9 \\
5.7 \\
4.6 \\
4.3\end{array}$ & $\begin{array}{r}1160000 \\
1050000 \\
159000 \\
120000 \\
80900 \\
43300 \\
76000 \\
37400 \\
18300 \\
21600 \\
4500000 \\
4560000 \\
42800 \\
41200 \\
91100 \\
40700 \\
13300 \\
25300 \\
25800 \\
294000 \\
370000 \\
7320000\end{array}$ & $\begin{array}{l}6.1 \\
6.0 \\
5.2 \\
5.1 \\
4.9 \\
4.6 \\
4.9 \\
4.6 \\
4.3 \\
4.3 \\
6.7 \\
6.7 \\
4.6 \\
4.6 \\
5.0 \\
4.6 \\
4.1 \\
4.4 \\
4.4 \\
5.5 \\
5.6 \\
6.9\end{array}$ \\
\hline
\end{tabular}


Table A-13. Resistance Readings (Ohms) Taken September' 19, 1988.

\begin{tabular}{lrlrlrl}
\hline $\begin{array}{l}\text { Depth } \\
\text { (in.) }\end{array}$ & \multicolumn{1}{c}{ Box } & Log & Box & Log & \multicolumn{1}{l}{ Box } & \multicolumn{1}{l}{ Log } \\
& $1 A$ & $1 A$ & $1 B$ & $1 B$ & $1 C$ & $1 C$ \\
\hline 2.0 & 139000 & 5.1 & 199000 & 5.3 & 701000 & 5.8 \\
4.0 & 423000 & 5.6 & 282000 & 5.5 & 673000 & 5.8 \\
6.0 & 320000 & 5.5 & 463000 & 5.7 & 216000 & 5.3 \\
8.0 & 27100 & 4.4 & 379000 & 5.6 & 159000 & 5.2 \\
10.0 & 99200 & 5.0 & 760000 & 5.9 & 106000 & 5.0 \\
12.0 & 89600 & 5.0 & 816000 & 5.9 & 100000 & 5.0 \\
14.0 & 73000 & 4.9 & 203000 & 5.3 & 102000 & 5.0 \\
16.0 & 44500 & 4.6 & 86900 & 4.9 & 78400 & 4.9 \\
18.0 & 16500 & 4.2 & 130000 & 5.1 & 23500 & 4.4 \\
20.0 & 210000 & 5.3 & 258000 & 5.4 & 28500 & 4.5 \\
22.0 & 207000 & 5.3 & 216000 & 5.3 & 3720000 & 6.6 \\
24.0 & 18600 & 4.3 & 7010000 & 6.8 & 3780000 & 6.6 \\
26.0 & 248000 & 5.4 & 7320000 & 6.9 & 100000 & 5.0 \\
28.0 & 246000 & 5.4 & 39200 & 4.6 & 103000 & 5.0 \\
30.0 & 20500 & 4.3 & 407000 & 5.6 & 130000 & 5.1 \\
32.0 & 16000 & 4.2 & 475000 & 5.7 & 89600 & 5.0 \\
34.0 & 266000 & 5.4 & 105000 & 5.0 & 16900 & 4.2 \\
36.0 & 268000 & 5.4 & 303000 & 5.5 & 34500 & 4.5 \\
38.0 & 29100 & 4.5 & 939000 & 6.0 & 33800 & 4.5 \\
40.0 & 26800 & 4.4 & 701000 & 5.8 & 306000 & 5.5 \\
42.0 & 17700 & 4.2 & 77200 & 4.9 & 353000 & 5.5 \\
44.0 & 96900 & 5.0 & 30600 & 4.5 & 4200000 & 6.6 \\
& & & & & &
\end{tabular}


Table A-14. Resistance Readings (Ohms) Taken September 19, 1988.

\begin{tabular}{lllllll}
\hline $\begin{array}{l}\text { Depth } \\
\text { (1n.) }\end{array}$ & $\begin{array}{l}\text { Box } \\
2 A\end{array}$ & $\begin{array}{l}\text { Log } \\
2 A\end{array}$ & $\begin{array}{l}\text { Box } \\
2 B\end{array}$ & $\begin{array}{l}\text { Log } \\
2 B\end{array}$ & $\begin{array}{l}\text { Box } \\
2 C\end{array}$ & $\begin{array}{l}\text { Log } \\
2 C\end{array}$ \\
\hline & & & & & & \\
2.0 & 279000 & 5.4 & 255000 & 5.4 & 267000 & 5.4 \\
4.0 & 95400 & 5.0 & 338000 & 5.5 & 201000 & 5.3 \\
6.0 & 38300 & 4.6 & 178000 & 5.3 & 92500 & 5.0 \\
8.0 & 26600 & 4.4 & 205000 & 5.3 & 83500 & 4.9 \\
10.0 & 20300 & 4.3 & 144000 & 5.2 & 84800 & 4.9 \\
12.0 & 18100 & 4.3 & 104000 & 5.0 & 34500 & 4.5 \\
14.0 & 17700 & 4.2 & 121000 & 5.1 & 23000 & 4.4 \\
16.0 & 18400 & 4.3 & 105000 & 5.0 & 18300 & 4.3 \\
18.0 & 15600 & 4.2 & 96900 & 5.0 & 15700 & 4.2 \\
20.0 & 12700 & 4.1 & 76000 & 4.9 & 15600 & 4.2 \\
22.0 & 13600 & 4.1 & 34100 & 4.5 & 16000 & 4.2 \\
24.0 & 14300 & 4.2 & 46900 & 4.7 & 13900 & 4.1 \\
26.0 & 13200 & 4.1 & 47500 & 4.7 & 11800 & 4.1 \\
28.0 & 14700 & 4.2 & 40700 & 4.6 & 29100 & 4.5 \\
30.0 & 15000 & 4.2 & 41700 & 4.6 & 32300 & 4.5 \\
32.0 & 15500 & 4.2 & 38800 & 4.6 & 17100 & 4.2 \\
34.0 & 21800 & 4.3 & 74700 & 4.9 & 15700 & 4.2 \\
36.0 & 24800 & 4.4 & 98500 & 5.0 & 14100 & 4.1 \\
38.0 & 24400 & 4.4 & 108000 & 5.0 & 13500 & 4.1 \\
40.0 & 19600 & 4.3 & 42300 & 4.6 & 12000 & 4.1 \\
42.0 & 15200 & 4.2 & 19600 & 4.3 & 10900 & 4.0 \\
44.0 & 15600 & 4.2 & 19200 & 4.3 & 9770 & 4.0 \\
& & & & & &
\end{tabular}


Table A-15. Resistarice Readings (Ohms) Taken September 21, 1988.

\begin{tabular}{|c|c|c|c|c|c|c|}
\hline $\begin{array}{l}\text { Depth } \\
\text { (in.) }\end{array}$ & $\begin{array}{l}\text { Box } \\
1 A\end{array}$ & $\begin{array}{l}\log \\
1 A\end{array}$ & $\begin{array}{l}\text { Box } \\
\text { lB }\end{array}$ & $\begin{array}{l}\log \\
1 B\end{array}$ & $\begin{array}{l}\text { Box } \\
1 C\end{array}$ & $\begin{array}{l}\log \\
1 C\end{array}$ \\
\hline $\begin{array}{l}2.0 \\
4.0 \\
6.0 \\
8.0 \\
10.0 \\
12.0 \\
14.0 \\
16.0 \\
18.0 \\
20.0 \\
22.0 \\
24.0 \\
26.0 \\
28.0 \\
30.0 \\
32.0 \\
34.0 \\
36.0 \\
38.0 \\
40.0 \\
42.0 \\
44.0\end{array}$ & $\begin{array}{r}169000 \\
918000 \\
803000 \\
29100 \\
124000 \\
116000 \\
87600 \\
85500 \\
17400 \\
263000 \\
255000 \\
19800 \\
736000 \\
760000 \\
21600 \\
16800 \\
439000 \\
433000 \\
31000 \\
28800 \\
184110 \\
80300\end{array}$ & $\begin{array}{l}5.2 \\
6.0 \\
5.9 \\
4.5 \\
5.1 \\
5.1 \\
4.9 \\
4.9 \\
4.2 \\
5.4 \\
5.4 \\
4.3 \\
5.9 \\
5.9 \\
4.3 \\
4.2 \\
5.6 \\
5.6 \\
4.5 \\
4.5 \\
4.3 \\
4.9\end{array}$ & $\begin{array}{r}175000 \\
330000 \\
580000 \\
397000 \\
835000 \\
889000 \\
216000 \\
93200 \\
139000 \\
267000 \\
248000 \\
9480000 \\
9930000 \\
43300 \\
457000 \\
600000 \\
113000 \\
334000 \\
1210000 \\
718000 \\
85500 \\
31600\end{array}$ & $\begin{array}{l}5.2 \\
5.5 \\
5.8 \\
5.6 \\
5.9 \\
5.9 \\
5.3 \\
5.0 \\
5.1 \\
5.4 \\
5.4 \\
7.0 \\
7.0 \\
4.6 \\
5.7 \\
5.8 \\
5.1 \\
5.5 \\
6.1 \\
5.9 \\
4.9 \\
4.5\end{array}$ & $\begin{array}{r}1740000 \\
1620000 \\
230000 \\
175000 \\
120000 \\
109000 \\
106000 \\
79700 \\
24600 \\
30600 \\
3310000 \\
3310000 \\
110000 \\
115000 \\
140000 \\
96200 \\
18000 \\
35700 \\
35300 \\
353000 \\
463000 \\
4040000\end{array}$ & $\begin{array}{l}6.2 \\
6.2 \\
5.4 \\
5.2 \\
5.1 \\
5.0 \\
5.0 \\
4.9 \\
4.4 \\
4.5 \\
6.5 \\
6.5 \\
5.0 \\
5.1 \\
5.1 \\
5.0 \\
4.3 \\
4.6 \\
4.5 \\
5.5 \\
5.7 \\
6.6\end{array}$ \\
\hline
\end{tabular}


Table A-16. Resistance Readings (Ohms) Taken September 21, 1988.

\begin{tabular}{lrrrrrr}
\hline $\begin{array}{l}\text { Depth } \\
\text { (in.) }\end{array}$ & $\begin{array}{c}\text { Box } \\
2 A\end{array}$ & $\begin{array}{l}\text { Log } \\
2 A\end{array}$ & $\begin{array}{c}\text { Box } \\
2 B\end{array}$ & $\begin{array}{l}\text { Log } \\
2 B\end{array}$ & $\begin{array}{l}\text { Box } \\
2 C\end{array}$ & $\begin{array}{l}\text { Log } \\
2 C\end{array}$ \\
\hline 2.0 & 261000 & 5.4 & 316000 & 5.5 & 210000 & 5.3 \\
4.0 & 112000 & 5.0 & 412000 & 5.6 & 244000 & 5.4 \\
6.0 & 42800 & 4.6 & 212000 & 5.3 & 110000 & 5.0 \\
8.0 & 29400 & 4.5 & 235000 & 5.4 & 97700 & 5.0 \\
10.0 & 22200 & 4.3 & 156000 & 5.2 & 95400 & 5.0 \\
12.0 & 19600 & 4.3 & 112000 & 5.0 & 37900 & 4.6 \\
14.0 & 19100 & 4.3 & 134000 & 5.1 & 24600 & 4.4 \\
16.0 & 19900 & 4.3 & 121000 & 5.1 & 19200 & 4.3 \\
18.0 & 16700 & 4.2 & 108000 & 5.0 & 16500 & 4.2 \\
20.0 & 13500 & 4.1 & 80900 & 4.9 & 16400 & 4.2 \\
22.0 & 14500 & 4.2 & 37400 & 4.6 & 16800 & 4.2 \\
24.0 & 15100 & 4.2 & 79000 & 4.9 & 14600 & 4.2 \\
26.0 & 14000 & 4.1 & 82900 & 4.9 & 12200 & 4.1 \\
28.0 & 15700 & 4.2 & 43900 & 4.6 & 32000 & 4.5 \\
30.0 & 16100 & 4.2 & 45100 & 4.7 & 35700 & 4.6 \\
32.0 & 16500 & 4.2 & 42800 & 4.6 & 17800 & 4.3 \\
34.0 & 23200 & 4.4 & 83500 & 4.9 & 16700 & 4.2 \\
36.0 & 26600 & 4.4 & 109000 & 5.0 & 15000 & 4.2 \\
38.0 & 26300 & 4.4 & 118000 & 5.1 & 14000 & 4.1 \\
40.0 & 21400 & 4.3 & 46300 & 4.7 & 12500 & 4.1 \\
42.0 & 16800 & 4.2 & 20700 & 4.3 & 11300 & 4.1 \\
44.0 & 15700 & 4.2 & 19800 & 4.3 & 10200 & 4.0 \\
& & & & & &
\end{tabular}


Table A-17. Resistance Readings (Ohms) Taken September 30, 1988.

\begin{tabular}{|c|c|c|c|c|c|c|}
\hline $\begin{array}{l}\text { Depth } \\
\text { (in.) }\end{array}$ & $\begin{array}{l}\text { Box } \\
1 A\end{array}$ & $\begin{array}{l}\log \\
1 A\end{array}$ & $\begin{array}{l}\text { Box } \\
1 B\end{array}$ & $\begin{array}{l}\log \\
1 B\end{array}$ & $\begin{array}{l}\text { Box } \\
1 C\end{array}$ & $\begin{array}{l}\log \\
1 C\end{array}$ \\
\hline $\begin{array}{l}2.0 \\
4.0 \\
6.0 \\
8.0 \\
10.0 \\
12.0 \\
14.0 \\
16.0 \\
18.0 \\
20.0 \\
22.0 \\
24.0 \\
26.0 \\
28.0 \\
30.0 \\
32.0 \\
34.0 \\
36.0 \\
38.0 \\
40.0 \\
42.0 \\
44.0\end{array}$ & $\begin{array}{r}268000 \\
889000 \\
742000 \\
29400 \\
129000 \\
123000 \\
97700 \\
91800 \\
17700 \\
246000 \\
237000 \\
20100 \\
712000 \\
7180 n 0 \\
22400 \\
17500 \\
402000 \\
402000 \\
32700 \\
30000 \\
19100 \\
68400\end{array}$ & $\begin{array}{l}5.4 \\
5.9 \\
5.9 \\
4.5 \\
5.1 \\
5.1 \\
5.0 \\
5.0 \\
4.2 \\
5.4 \\
5.4 \\
4.3 \\
5.9 \\
5.9 \\
4.4 \\
4.2 \\
5.6 \\
5.6 \\
4.5 \\
4.5 \\
4.3 \\
4.8\end{array}$ & $\begin{array}{r}251000 \\
330000 \\
585000 \\
397000 \\
790000 \\
855000 \\
210000 \\
94700 \\
143000 \\
267000 \\
251000 \\
9560000 \\
9840000 \\
46300 \\
43300 \\
489000 \\
117000 \\
330000 \\
1310000 \\
822000 \\
91800 \\
33400\end{array}$ & $\begin{array}{l}5.4 \\
5.5 \\
5.8 \\
5.6 \\
5.9 \\
5.9 \\
5.3 \\
5.0 \\
5.2 \\
5.4 \\
5.4 \\
7.0 \\
7.0 \\
4.7 \\
4.6 \\
5.7 \\
5.1 \\
5.5 \\
6.1 \\
5.9 \\
5.0 \\
: .5\end{array}$ & $\begin{array}{r}1670000 \\
1510000 \\
228000 \\
174000 \\
122000 \\
112000 \\
108000 \\
79700 \\
25800 \\
32700 \\
3130000 \\
3110000 \\
117000 \\
124000 \\
151000 \\
103000 \\
19400 \\
37900 \\
36100 \\
345000 \\
457000 \\
3260000\end{array}$ & $\begin{array}{l}6.2 \\
6.2 \\
5.4 \\
5.2 \\
5.1 \\
5.0 \\
5.0 \\
4.9 \\
4.4 \\
4.5 \\
6.5 \\
6.5 \\
5.1 \\
5.1 \\
5.2 \\
5.0 \\
4.3 \\
4.6 \\
4.6 \\
5.5 \\
5.7 \\
6.5\end{array}$ \\
\hline
\end{tabular}


Table A-18. Reststance Readings (Ohms) Taken September 30, 1988.

\begin{tabular}{lllllll}
\hline $\begin{array}{l}\text { Depth } \\
\text { th. }\end{array}$ & $\begin{array}{l}\text { Box } \\
\text { IA }\end{array}$ & $\begin{array}{l}\text { Log } \\
2 A\end{array}$ & $\begin{array}{l}\text { Box } \\
2 B\end{array}$ & $\begin{array}{l}\text { Log } \\
2 B\end{array}$ & $\begin{array}{l}\text { Box } \\
2 C\end{array}$ & $\begin{array}{l}\text { Log } \\
2 C\end{array}$ \\
\hline 2.0 & 327000 & 5.5 & 349000 & 5.5 & 210000 & 5.3 \\
4.0 & 111000 & 5.0 & 417000 & 5.6 & 244000 & 5.4 \\
6.0 & 41200 & 4.6 & 214000 & 5.3 & 103000 & 5.0 \\
8.0 & 28500 & 4.5 & 239000 & 5.4 & 91800 & 5.0 \\
10.0 & 21600 & 4.3 & 160000 & 5.2 & 96900 & 5.0 \\
12.0 & 19200 & 4.3 & 115000 & 5.1 & 38800 & 4.6 \\
14.0 & 19100 & 4.3 & 140000 & 5.1 & 25300 & 4.4 \\
16.0 & 20100 & 4.3 & 127000 & 5.1 & 19800 & 4.3 \\
18.0 & 16900 & 4.2 & 114000 & 5.1 & 16900 & 4.2 \\
20.0 & 13800 & 4.1 & 85500 & 4.9 & 17100 & 4.2 \\
22.0 & 15000 & 4.2 & 40700 & 4.6 & 17500 & 4.2 \\
24.0 & 15700 & 4.2 & 84800 & 4.9 & 15200 & 4.2 \\
26.0 & 14600 & 4.2 & 88200 & 4.9 & 12800 & 4.1 \\
28.0 & 16200 & 4.2 & 78400 & 4.9 & 34500 & 4.5 \\
30.0 & 16700 & 4.2 & 76600 & 4.9 & 38800 & 4.6 \\
32.0 & 17100 & 4.2 & 46900 & 4.7 & 18800 & 4.3 \\
34.0 & 23900 & 4.4 & 92500 & 5.0 & 17500 & 4.2 \\
36.0 & 27400 & 4.4 & 120000 & 5.1 & 15700 & 4.2 \\
38.0 & 27100 & 4.4 & 124000 & 5.1 & 14700 & 4.2 \\
40.0 & 22000 & 4.3 & 72400 & 4.9 & 13100 & 4.1 \\
42.0 & 17100 & 4.2 & 23500 & 4.4 & 11900 & 4.1 \\
44.0 & 16200 & 4.2 & 22200 & 4.3 & 10600 & 4.0 \\
& & & & & &
\end{tabular}


Table A-19. Resistance Readings (Ohms) Taken October 3, 1988 (4-Hour Postrainulator).

\begin{tabular}{|c|c|c|c|c|c|c|}
\hline $\begin{array}{l}\text { Depth } \\
(t n .)\end{array}$ & $\begin{array}{l}\text { Box } \\
1 A\end{array}$ & $\begin{array}{l}\log \\
I A\end{array}$ & $\begin{array}{l}\text { Box } \\
1 B\end{array}$ & $\begin{array}{l}\log \\
1 B\end{array}$ & $\begin{array}{l}\text { Box } \\
1 C\end{array}$ & $\begin{array}{l}\log \\
1 C\end{array}$ \\
\hline $\begin{array}{l}2.0 \\
4.0 \\
6.0 \\
8.0 \\
10.0 \\
12.0 \\
14.0 \\
16.0 \\
18.0 \\
20.0 \\
22.0 \\
24.0 \\
26.0 \\
28.0 \\
30.0 \\
32.0 \\
34.0 \\
36.0 \\
38.0 \\
40.0 \\
42.0 \\
44.0\end{array}$ & $\begin{array}{r}31000 \\
402000 \\
383000 \\
26100 \\
121000 \\
117000 \\
94700 \\
88200 \\
16800 \\
212000 \\
207000 \\
19100 \\
566000 \\
502000 \\
21200 \\
16700 \\
357000 \\
366000 \\
30300 \\
27900 \\
18800 \\
101000\end{array}$ & $\begin{array}{l}4.5 \\
5.6 \\
5.6 \\
4.4 \\
5.1 \\
5.1 \\
5.0 \\
4.9 \\
4.2 \\
5.3 \\
5.3 \\
4.3 \\
5.8 \\
5.7 \\
4.3 \\
4.2 \\
5.6 \\
5.6 \\
4.5 \\
4.4 \\
4.3 \\
5.0\end{array}$ & $\begin{array}{r}73600 \\
90300 \\
224000 \\
285000 \\
712000 \\
778000 \\
199000 \\
89600 \\
133000 \\
274000 \\
232000 \\
8120000 \\
7570000 \\
43300 \\
357000 \\
407000 \\
111000 \\
300000 \\
1180000 \\
784000 \\
86100 \\
33000\end{array}$ & $\begin{array}{l}4.9 \\
5.0 \\
5.4 \\
5.5 \\
5.9 \\
5.9 \\
5.3 \\
5.0 \\
5.1 \\
5.4 \\
5.4 \\
6.9 \\
6.9 \\
4.6 \\
5.6 \\
5.6 \\
5.0 \\
5.5 \\
6.1 \\
5.9 \\
4.9 \\
4.5\end{array}$ & $\begin{array}{r}1210000 \\
1130000 \\
203000 \\
167000 \\
114000 \\
106000 \\
102000 \\
76600 \\
24100 \\
30600 \\
\\
112000 \\
117000 \\
139000 \\
97700 \\
18800 \\
35300 \\
34100 \\
285000 \\
379000 \\
7320000\end{array}$ & $\begin{array}{l}6.1 \\
6.1 \\
5.3 \\
5.2 \\
5.1 \\
5.0 \\
5.0 \\
4.9 \\
4.4 \\
4.5 \\
\text { ERR } \\
\text { ERR } \\
5.0 \\
5.1 \\
5.1 \\
5.0 \\
4.3 \\
4.5 \\
4.5 \\
5.5 \\
5.6 \\
6.9\end{array}$ \\
\hline
\end{tabular}


Table A-20. Reststance Readings (Ohms) Taken October 4, 1988 (24-Hour Postratnulator).

\begin{tabular}{|c|c|c|c|c|c|c|}
\hline $\begin{array}{l}\text { Depth } \\
(\operatorname{tn} .)\end{array}$ & $\begin{array}{l}B 0 x \\
\text { IA }\end{array}$ & $\begin{array}{l}\log \\
1 A\end{array}$ & $\begin{array}{l}B 0 X \\
1 B\end{array}$ & $\begin{array}{l}\log \\
1 B\end{array}$ & $\begin{array}{l}\text { Box } \\
1 C\end{array}$ & $\begin{array}{l}\log \\
1 \mathrm{C}\end{array}$ \\
\hline $\begin{array}{l}2.0 \\
4.0 \\
6.0 \\
8.0 \\
10.0 \\
12.0 \\
14.0 \\
16.0 \\
18.0 \\
20.0 \\
22.0 \\
24.0 \\
26.0 \\
28.0 \\
30.0 \\
32.0 \\
34.0 \\
36.0 \\
38.0 \\
40.0 \\
42.0 \\
44.0\end{array}$ & $\begin{array}{r}106000 \\
668000 \\
615000 \\
27400 \\
127000 \\
124000 \\
99200 \\
92500 \\
16800 \\
212000 \\
207000 \\
19100 \\
561000 \\
502000 \\
21200 \\
16500 \\
370000 \\
379000 \\
30300 \\
27900 \\
18600 \\
76600\end{array}$ & $\begin{array}{l}5.0 \\
5.8 \\
5.8 \\
4.4 \\
5.1 \\
5.1 \\
5.0 \\
5.0 \\
4.2 \\
5.3 \\
5.3 \\
4.3 \\
5.7 \\
5.7 \\
4.3 \\
4.2 \\
5.6 \\
5.6 \\
4.5 \\
4.4 \\
4.3 \\
4.9\end{array}$ & $\begin{array}{r}111000 \\
160000 \\
300000 \\
303000 \\
718000 \\
772000 \\
198000 \\
90300 \\
13300 \\
274000 \\
230000 \\
9560000 \\
\\
43300 \\
357000 \\
402000 \\
108000 \\
310000 \\
1180000 \\
790000 \\
88200 \\
32300\end{array}$ & $\begin{array}{l}5.0 \\
5.2 \\
5.5 \\
5.5 \\
5.9 \\
5.9 \\
5.3 \\
5.0 \\
4.1 \\
5.4 \\
5.4 \\
7.0 \\
\text { E.RR } \\
4.6 \\
5.6 \\
5.6 \\
5.0 \\
5.5 \\
6.1 \\
5.9 \\
4.9 \\
4.5\end{array}$ & $\begin{array}{r}1450000 \\
1310000 \\
224000 \\
177000 \\
116000 \\
106000 \\
102000 \\
48200 \\
23200 \\
30000 \\
8960000 \\
8410000 \\
112000 \\
118000 \\
144000 \\
98500 \\
18800 \\
34900 \\
33400 \\
291000 \\
383000 \\
7250000\end{array}$ & $\begin{array}{l}6.2 \\
6.1 \\
5.4 \\
5.2 \\
5.1 \\
5.0 \\
5.0 \\
4.7 \\
4.4 \\
4.5 \\
7.0 \\
6.9 \\
5.0 \\
5.1 \\
5.2 \\
5.0 \\
4.3 \\
4.5 \\
4.5 \\
5.5 \\
5.6 \\
6.9\end{array}$ \\
\hline
\end{tabular}


WHC' - F.P. 0299

\section{APPENDIX B}

TEST \ GRAPHS

B-1/Bii 
WHC-EP-0299

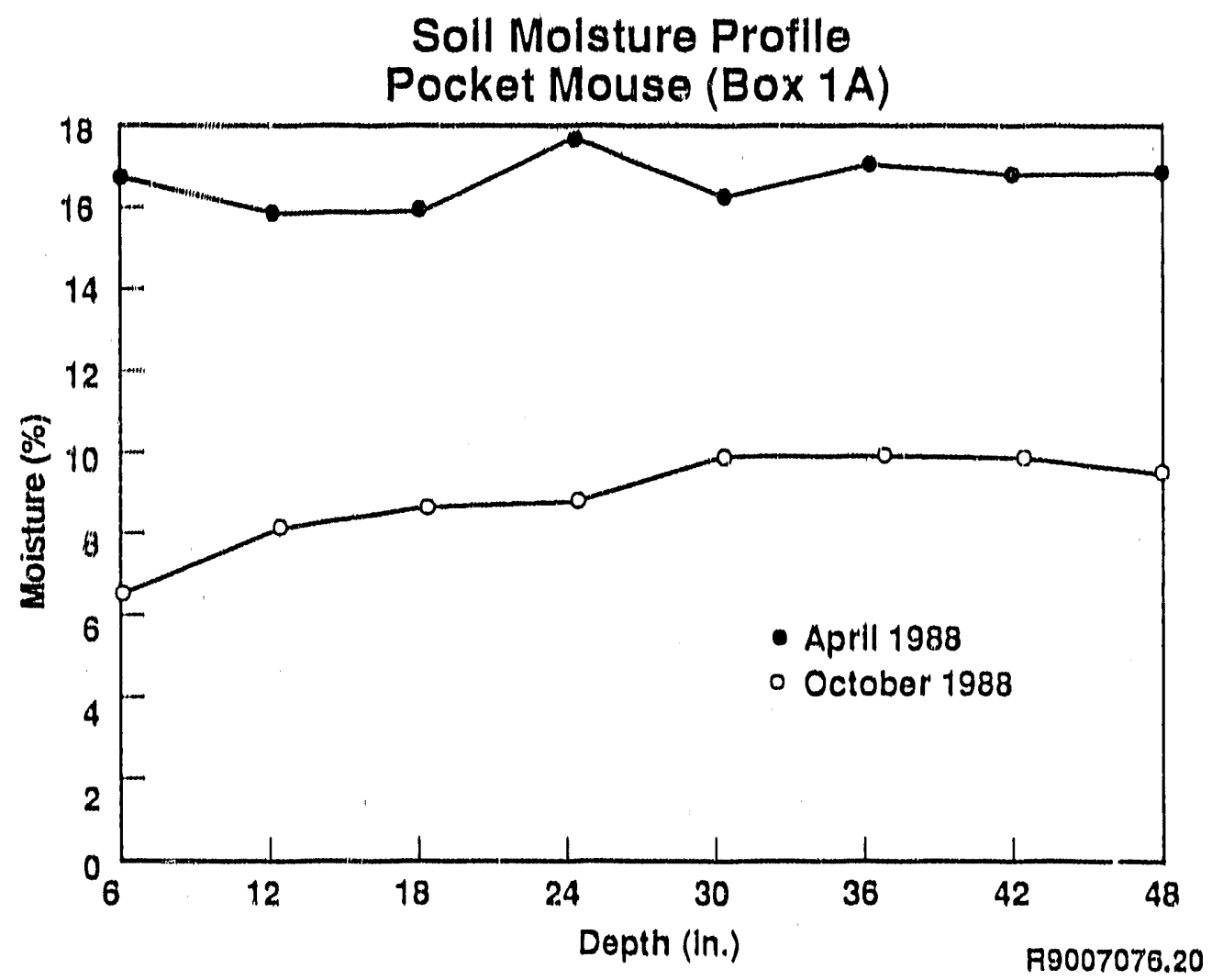

Soll Moistu re Profile

Control (Box 1B)

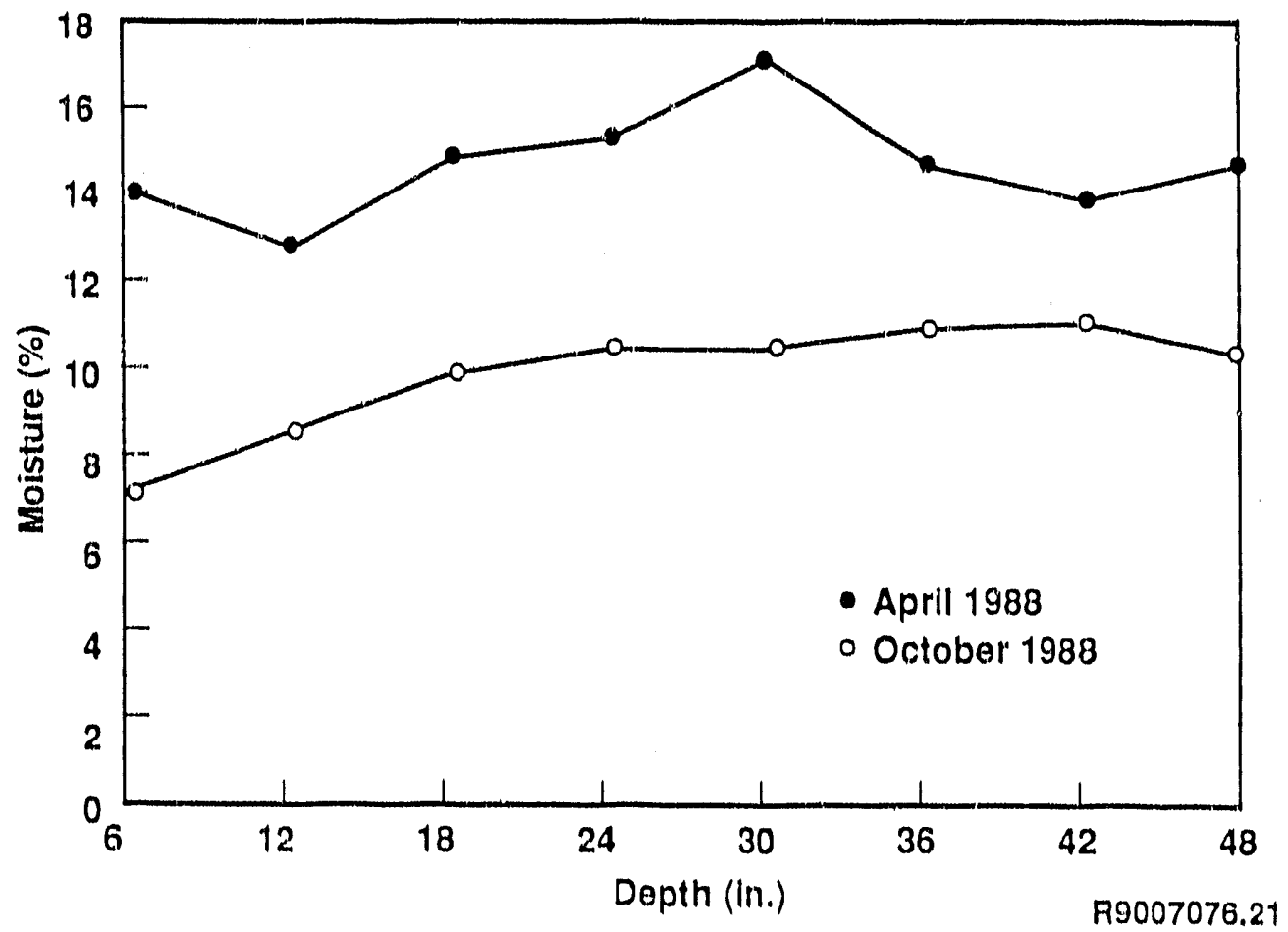


WHC-EP-0299

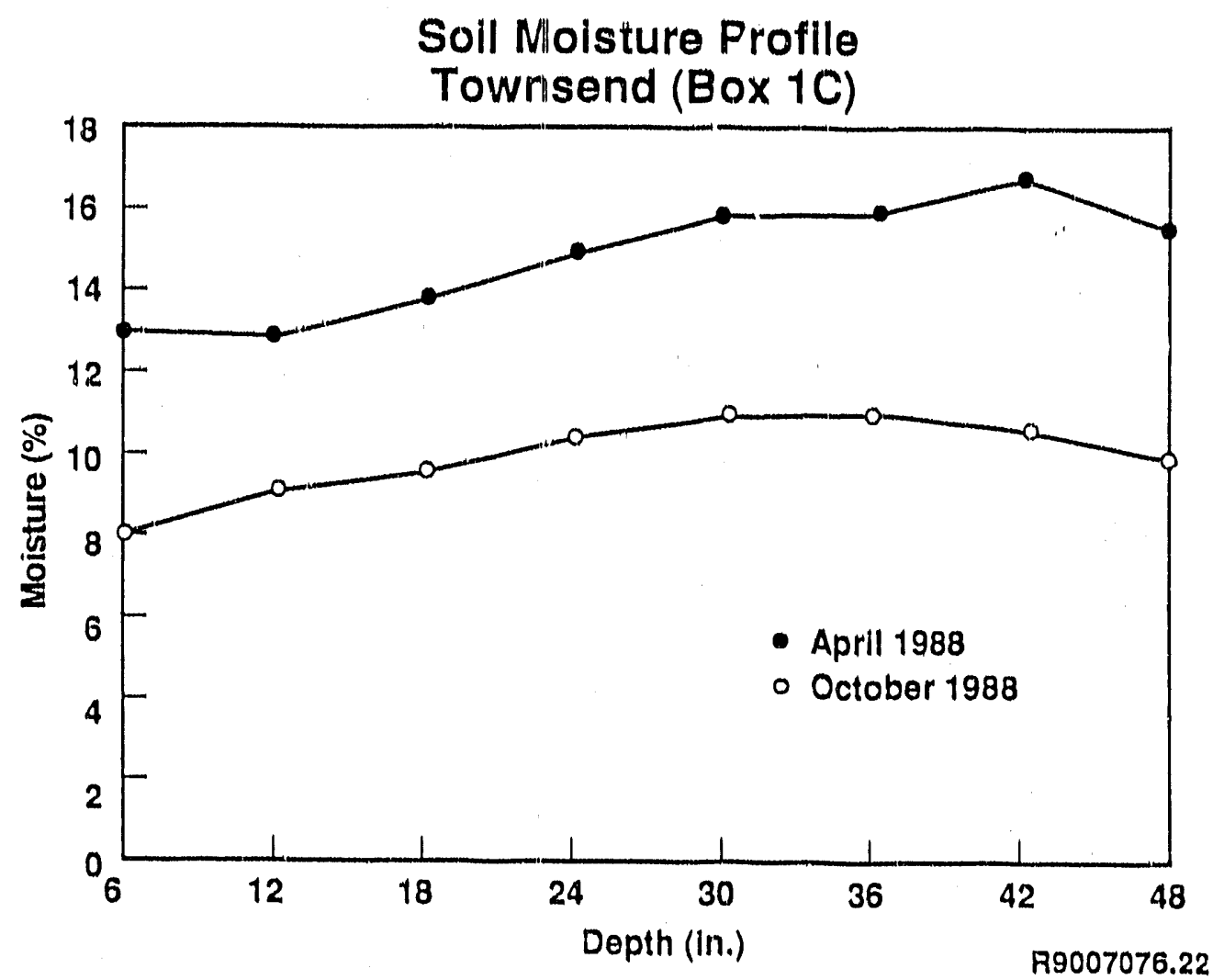

Soil Moisture Profile

Control (Box 2A)

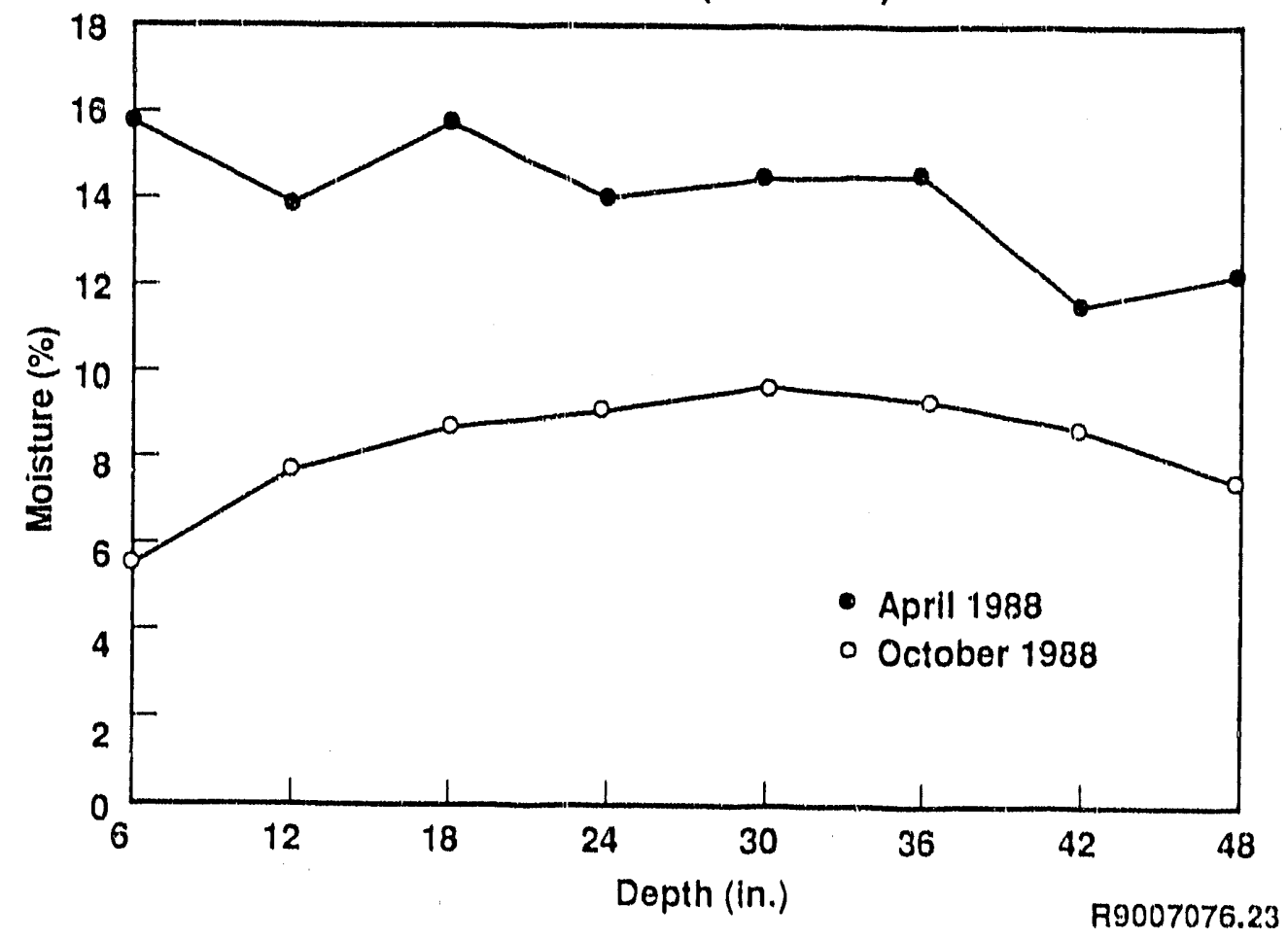


WHC-EP-0299

Soil Moisture Profile

Townsend (Box 2B)

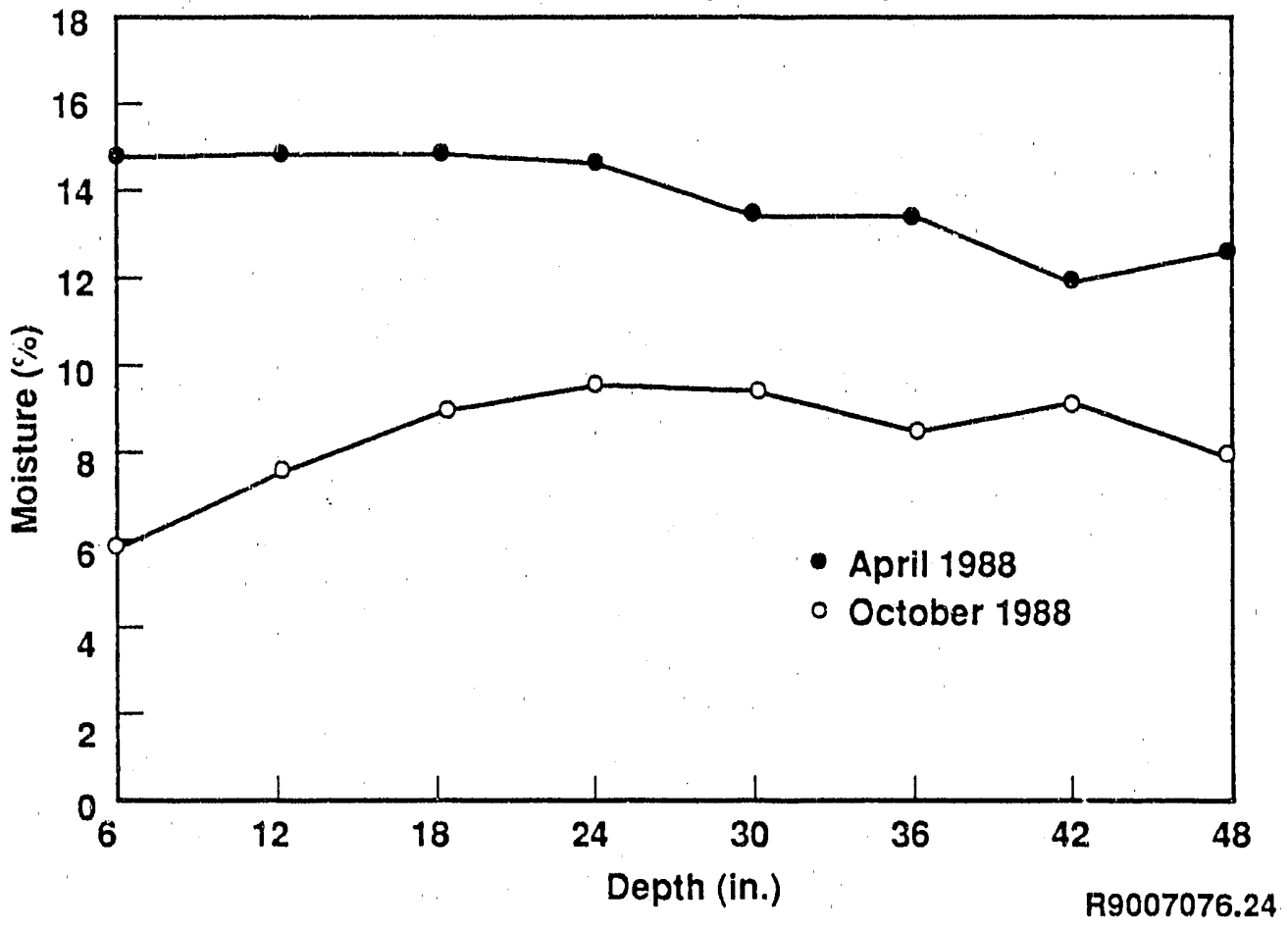

Soil Moisture Profile

Pocket Mouse (Box 2C)

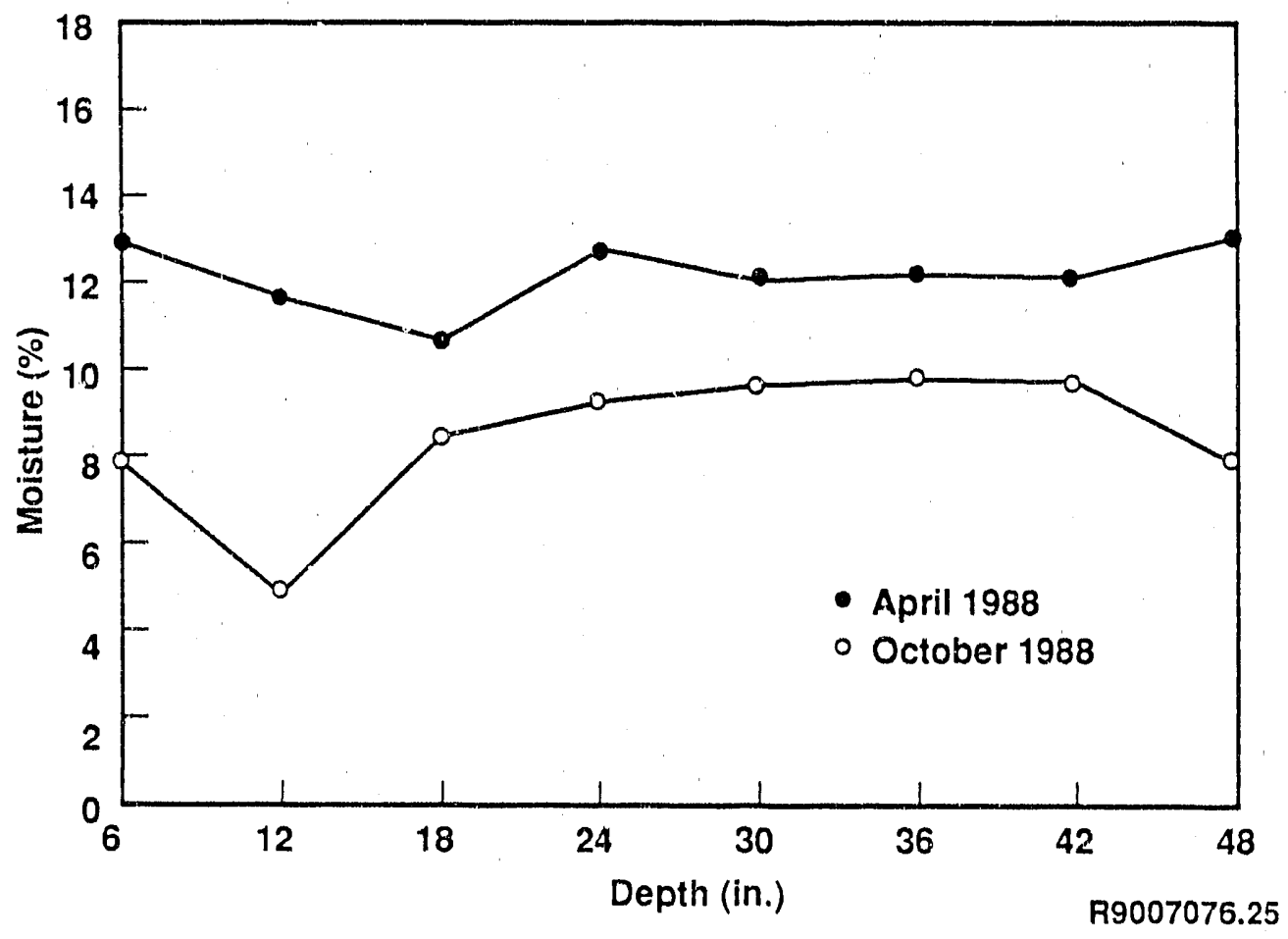


WHC-EP-0299

Soil Moisture Profile

April to October 1988 (0-6 in.)

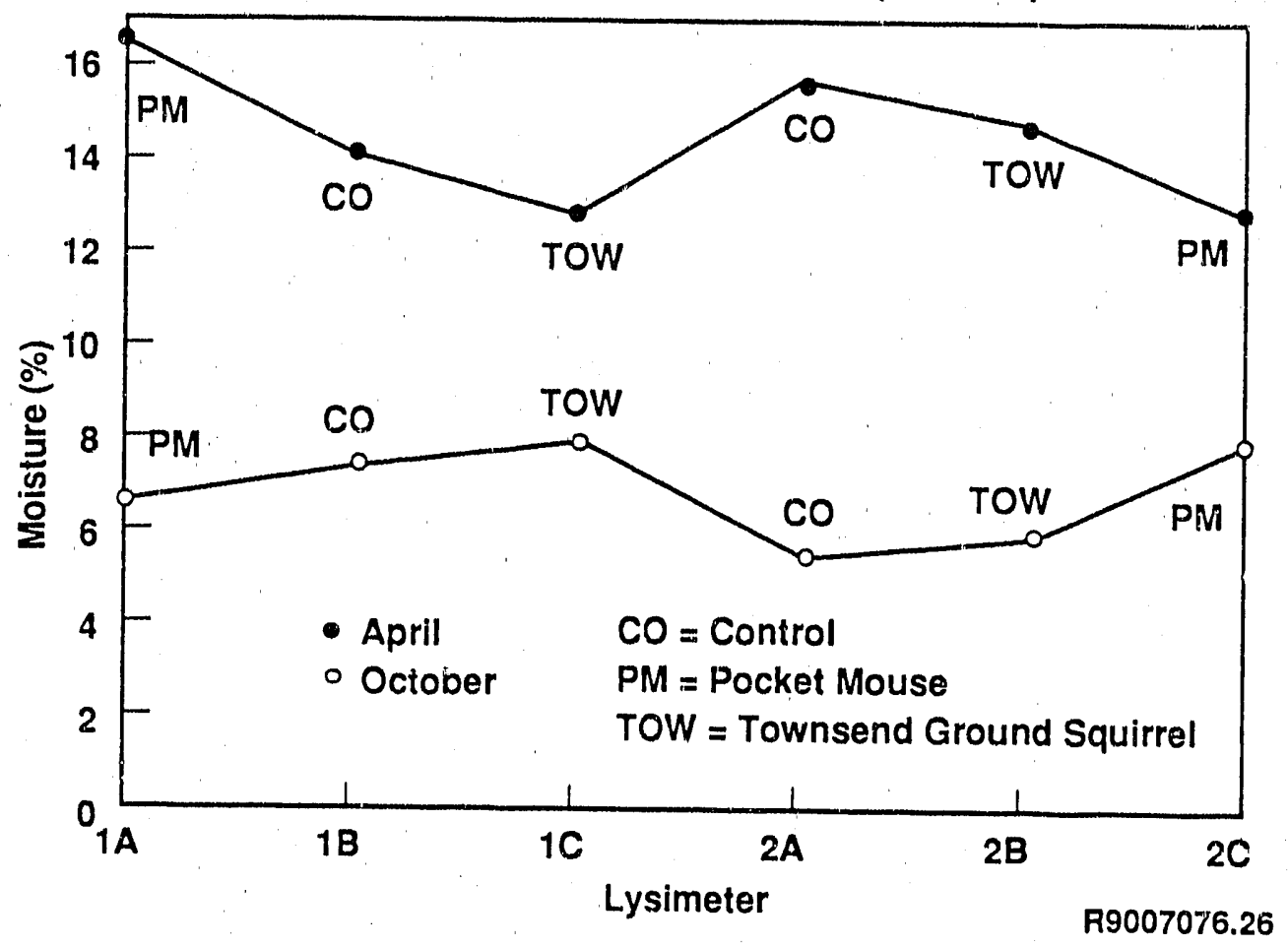

Soil N'oisture Profile

April to October 1988 (6-12 in.)

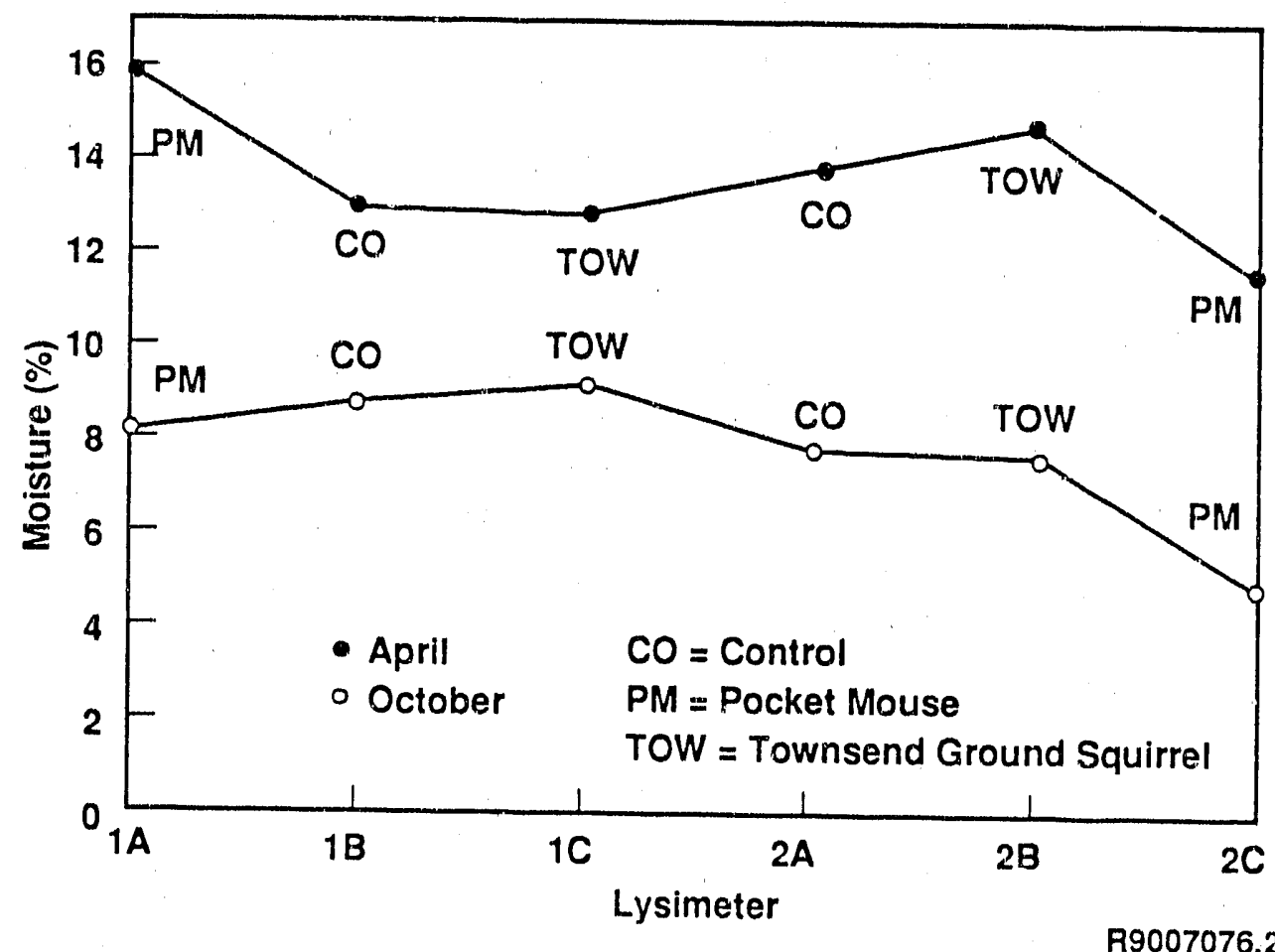

R9007076.27 
WHC-EP-0299

Soil Moisture Profile

April to October 1988 (12-18 in.)

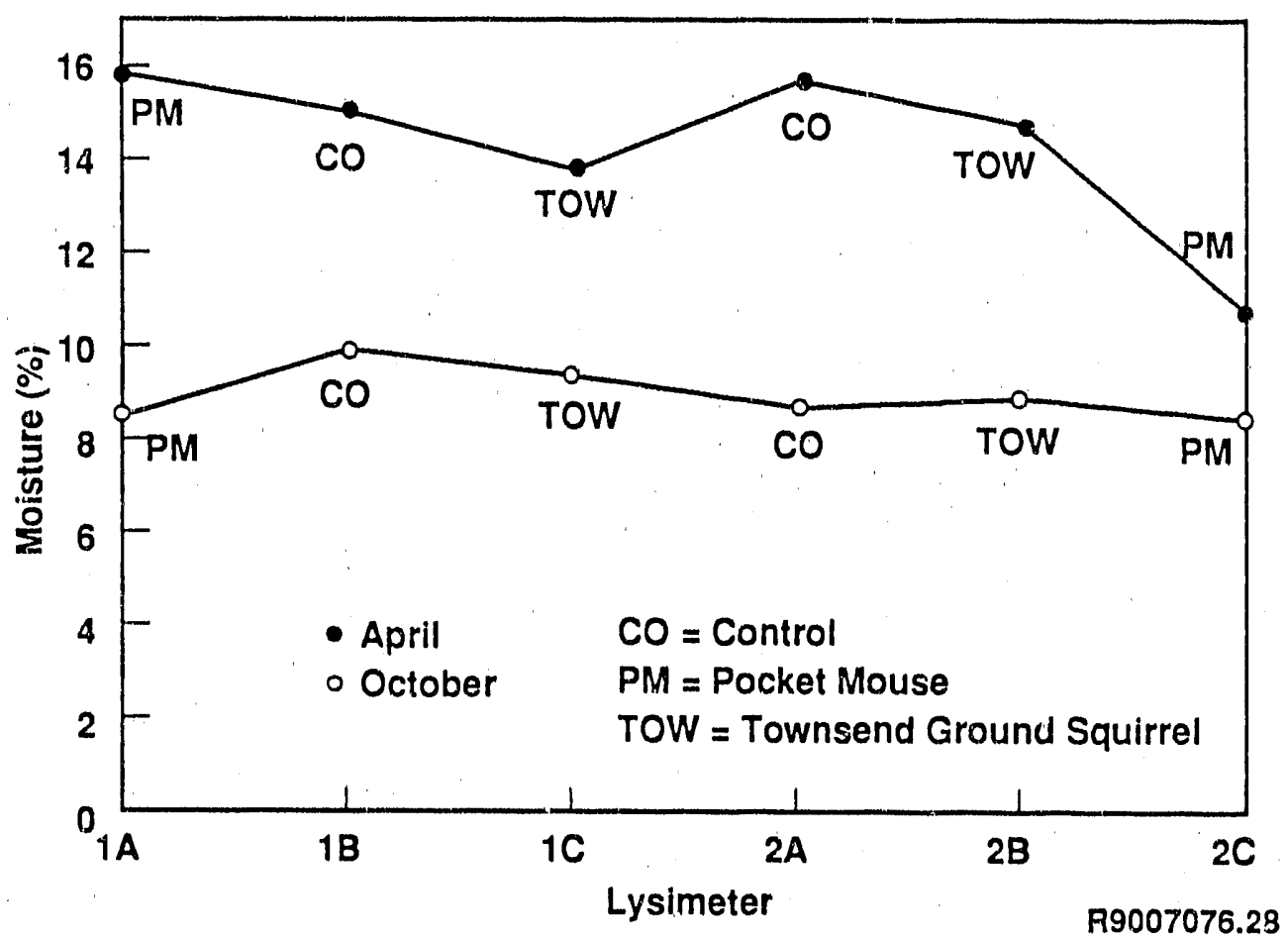

Soil Moisture Profile

April to October 1988 ; 18-24 in.)

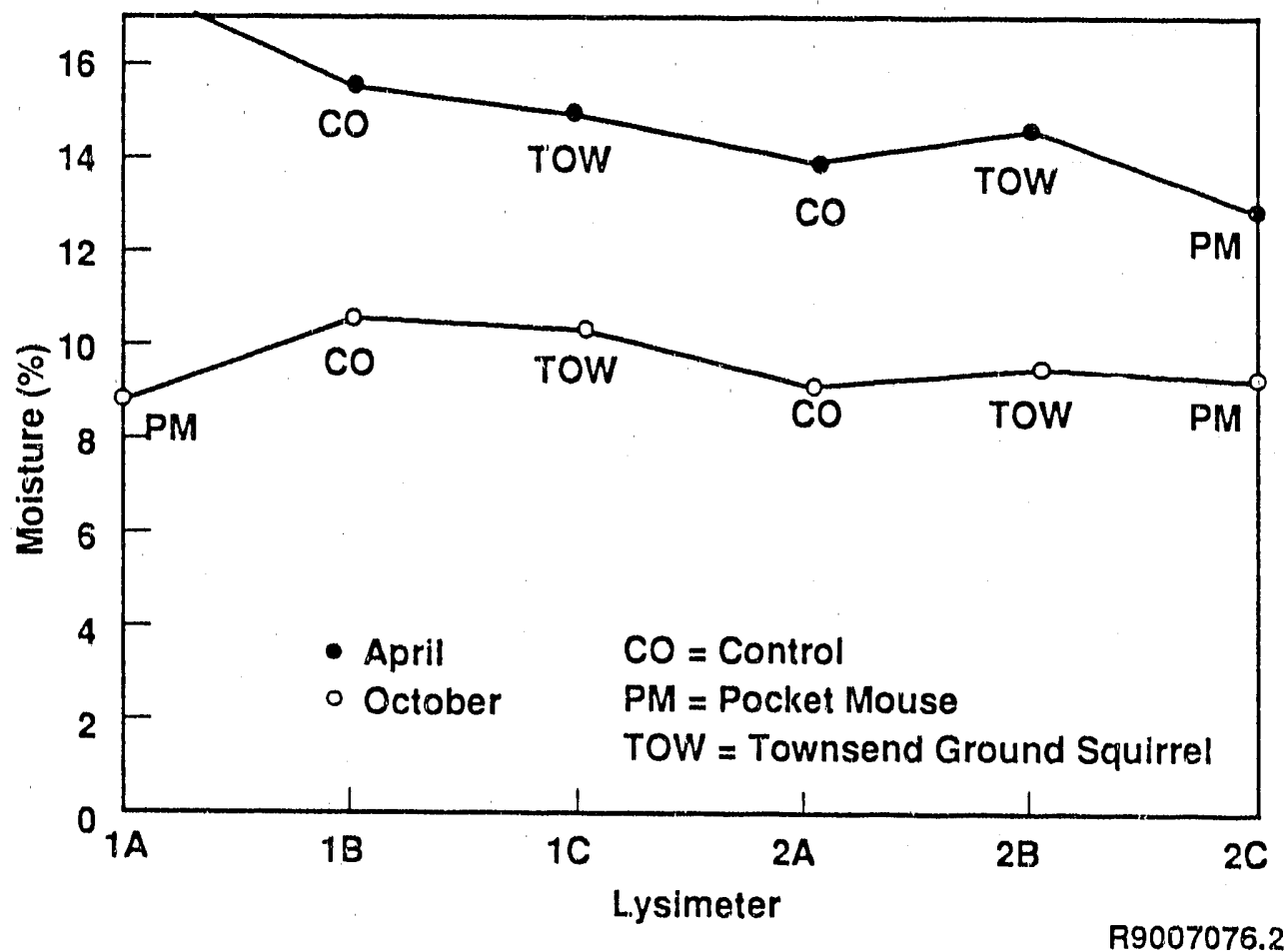


WHC-EP-0299

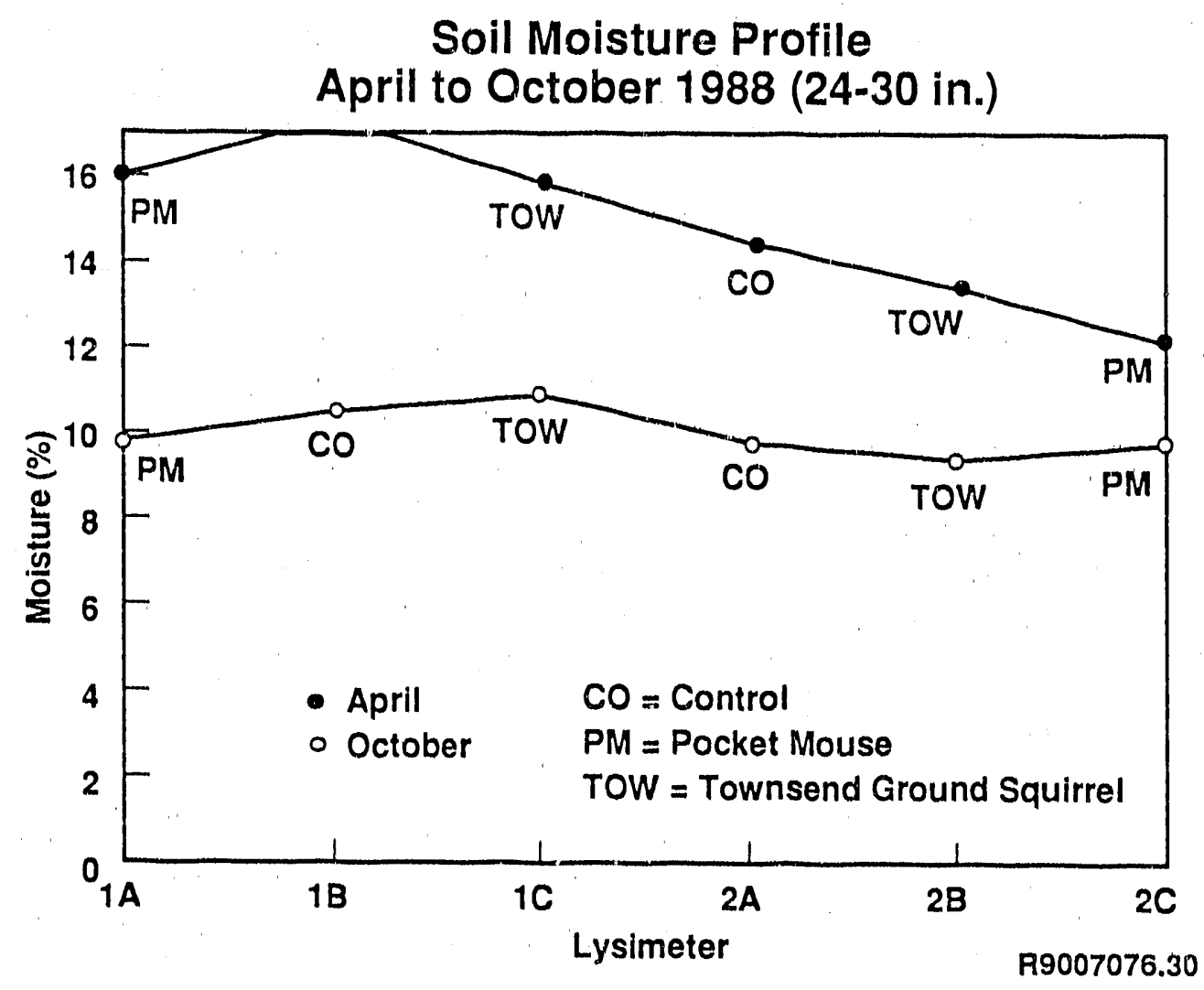

Soil Moisture Profile

April to October 1988 (30-36 in.)

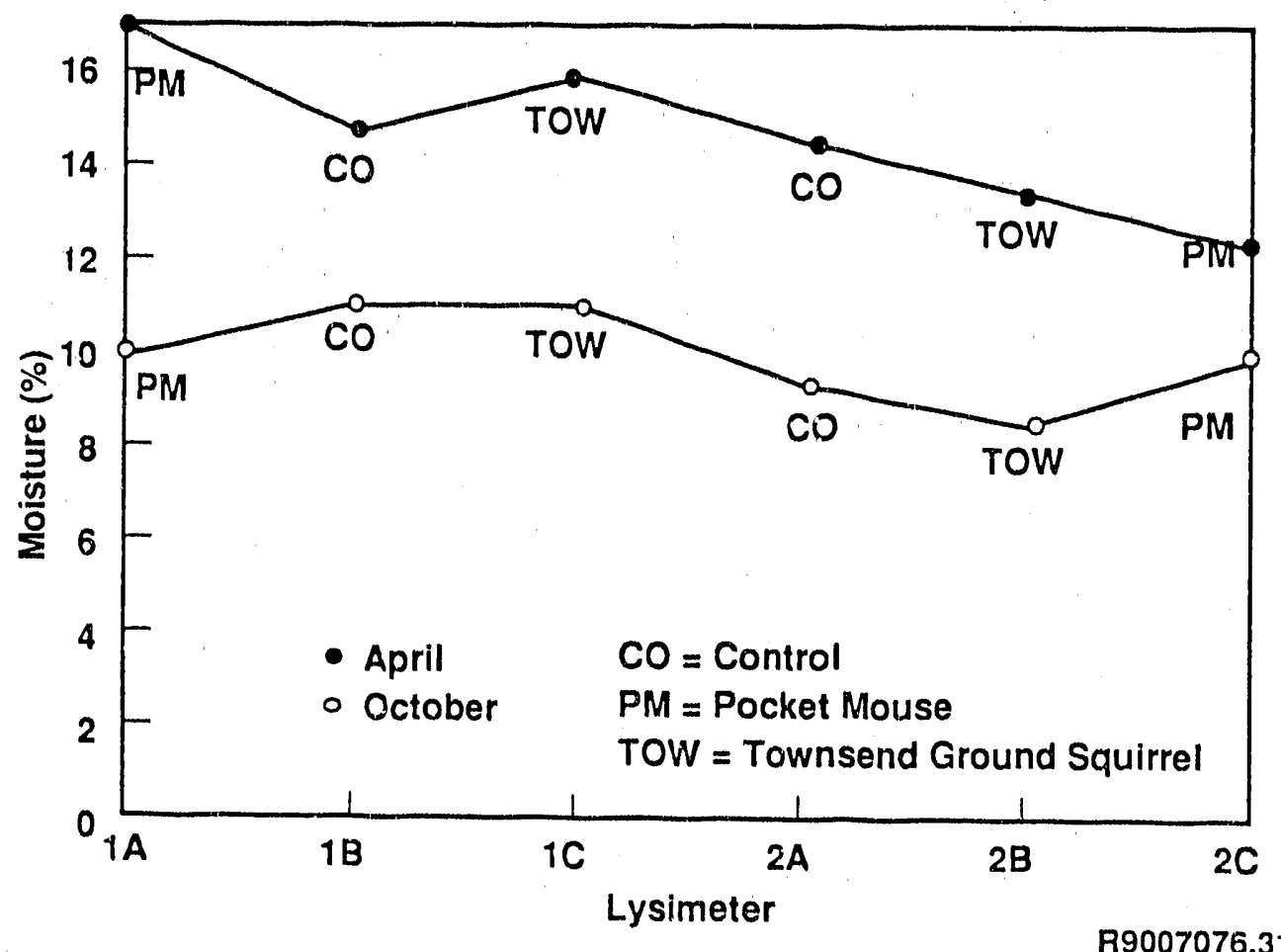


Soil Moisture Profile

April to October 1988 (36-42 in.)

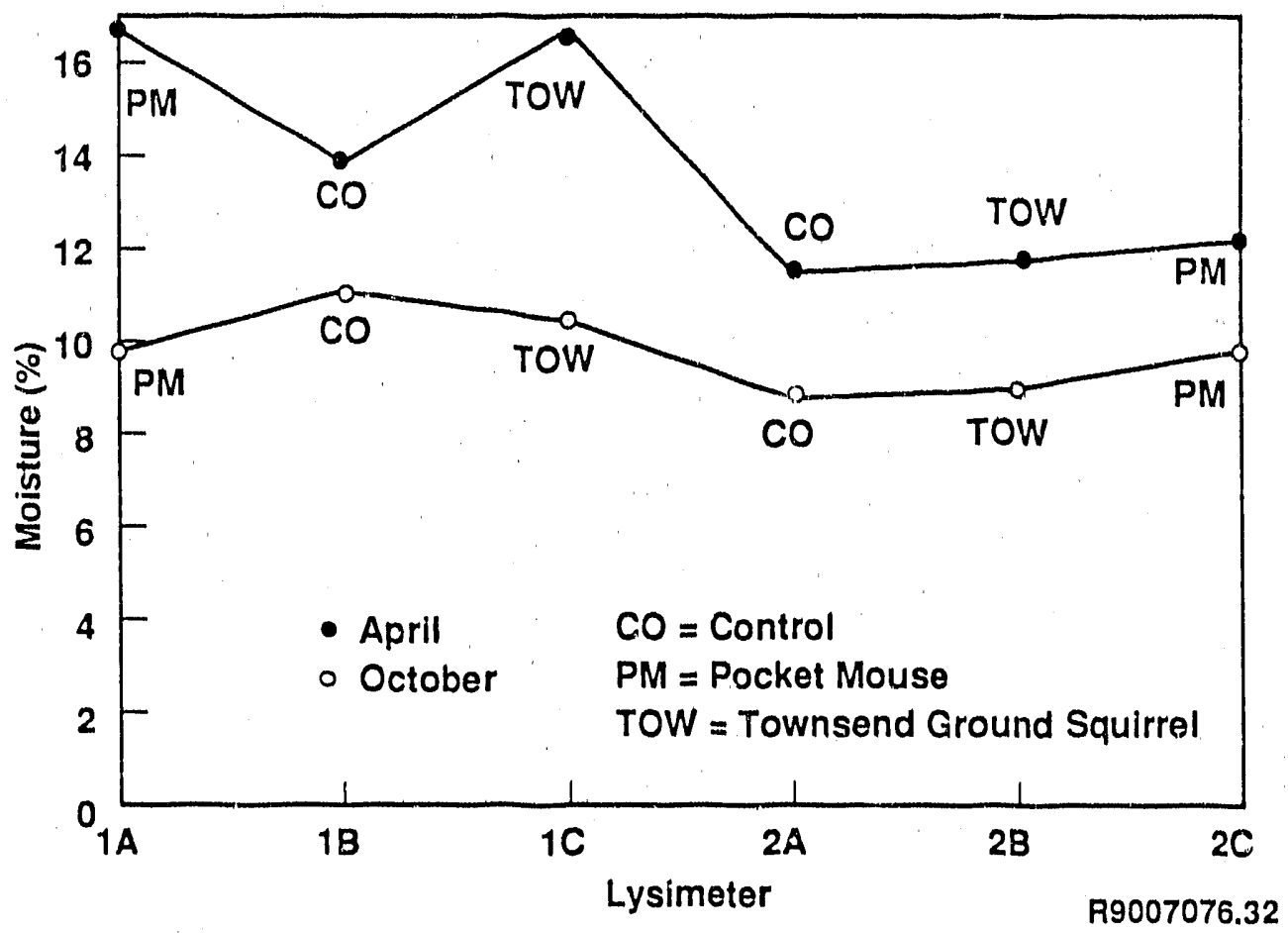

Soil Moisture Profile

April to October 1988 (42.48 in.)

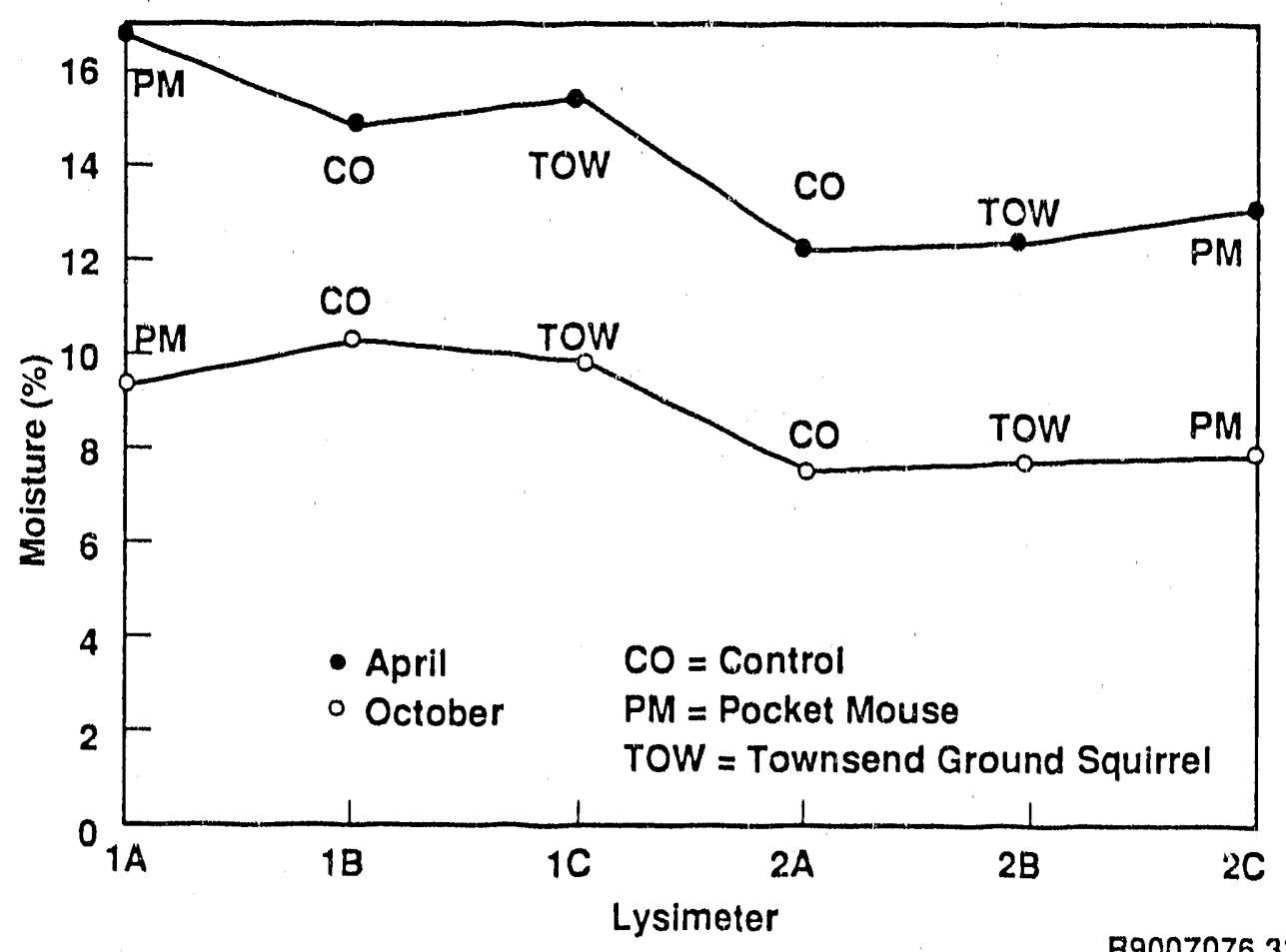


WHC-EP-0299
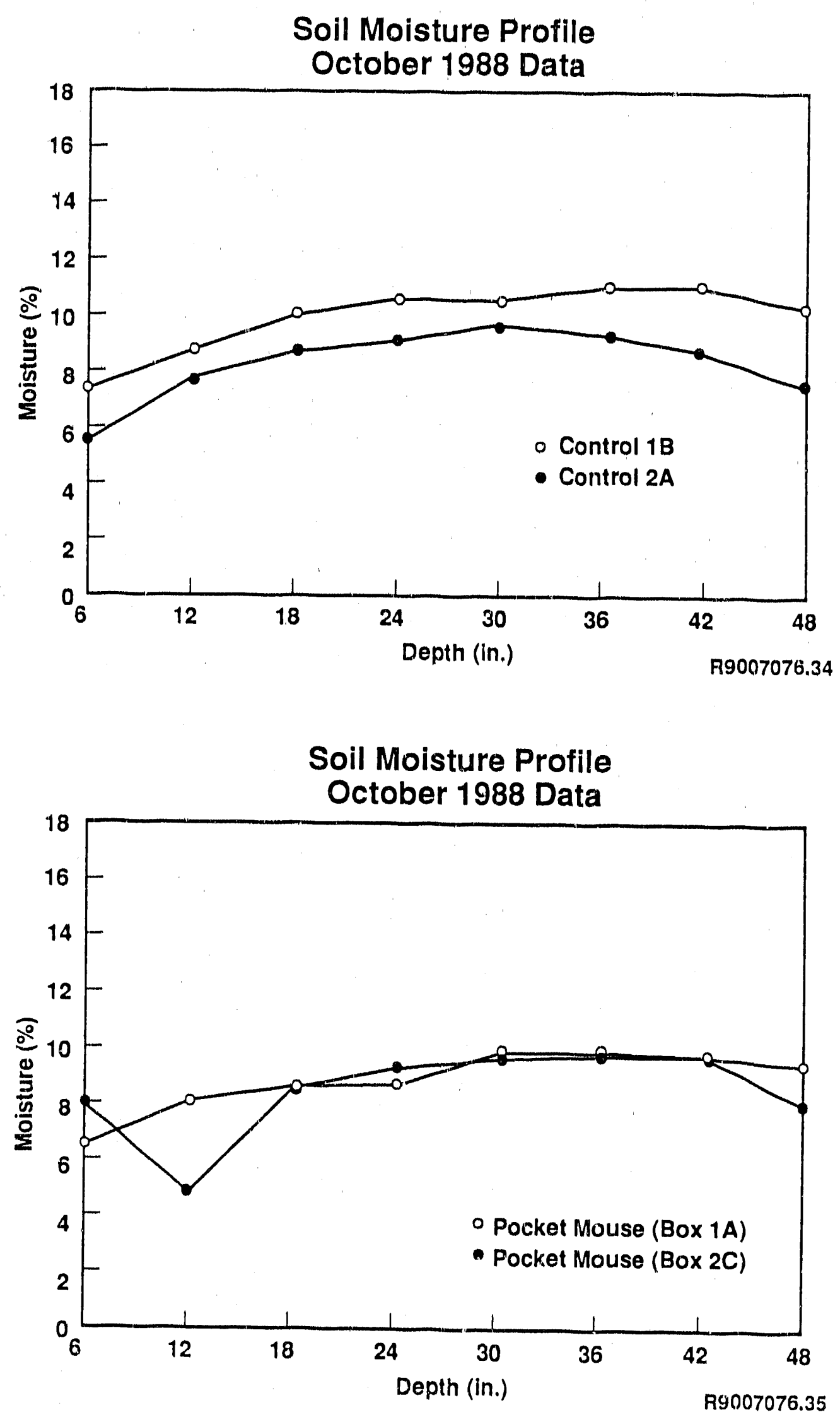
WHC-EP-0299

Soil Moisture Profile

October 1988 Data

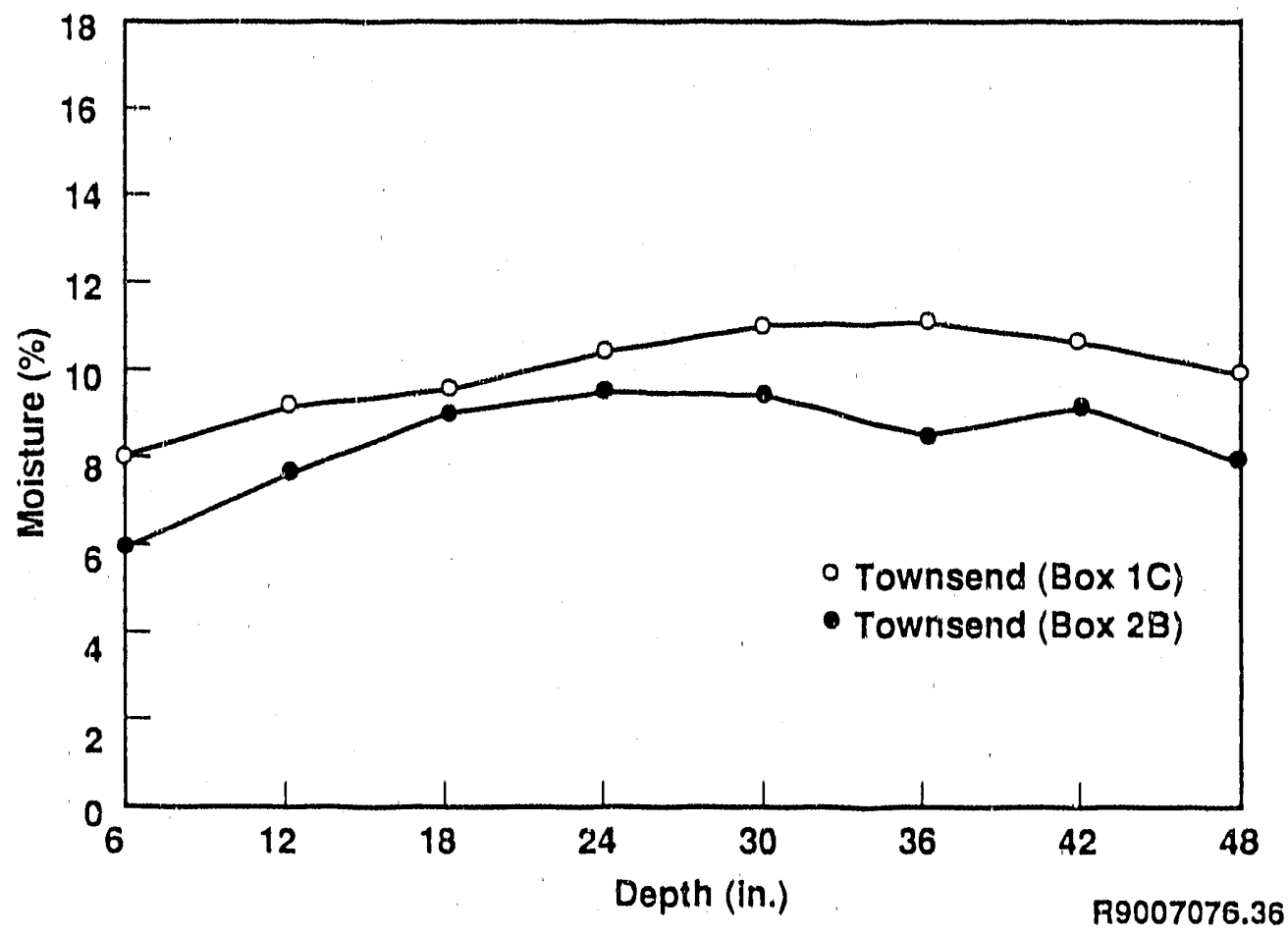

Soil Moisture Profile

October 1988 Readings

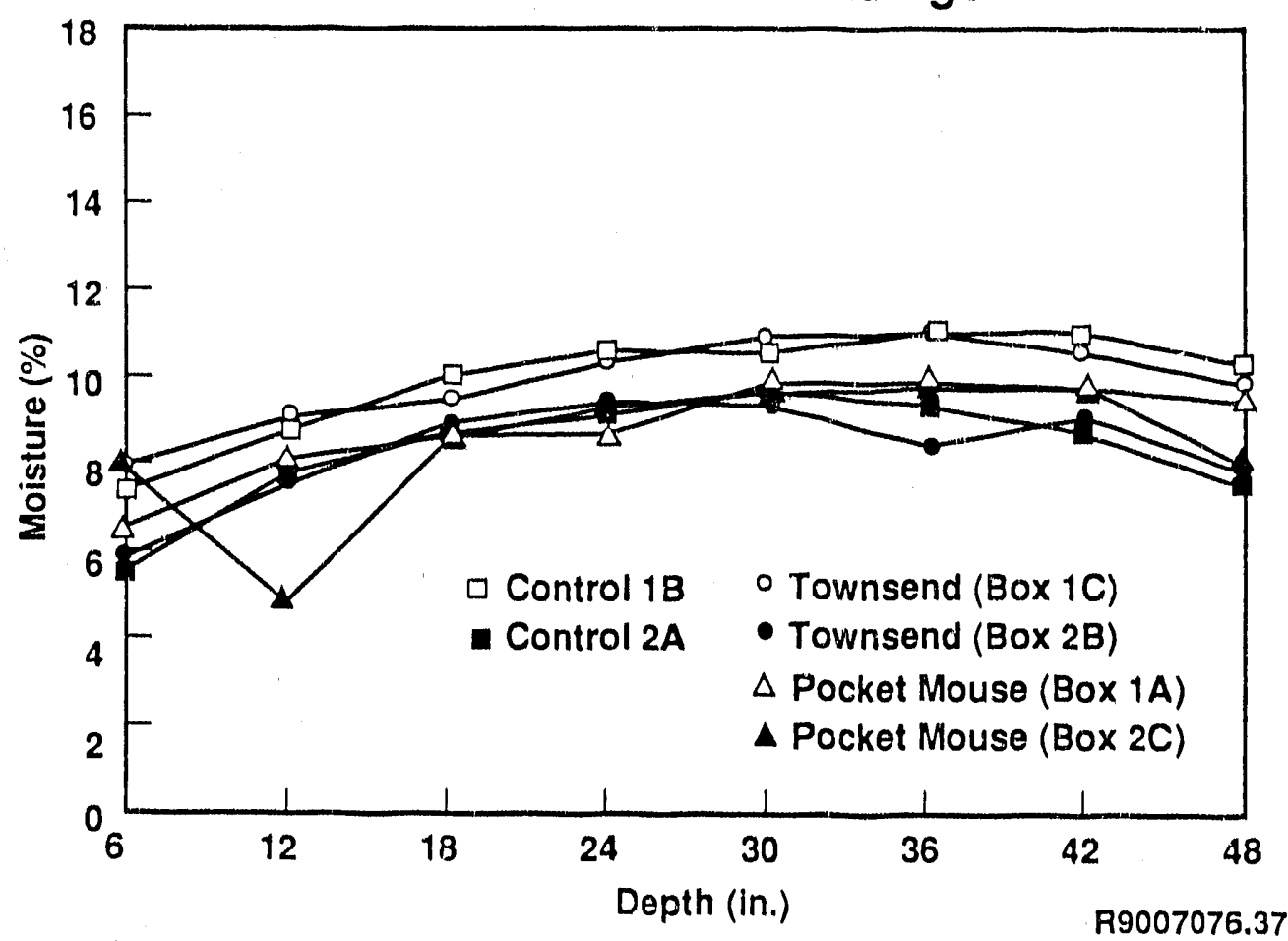


WHC-EP-0299
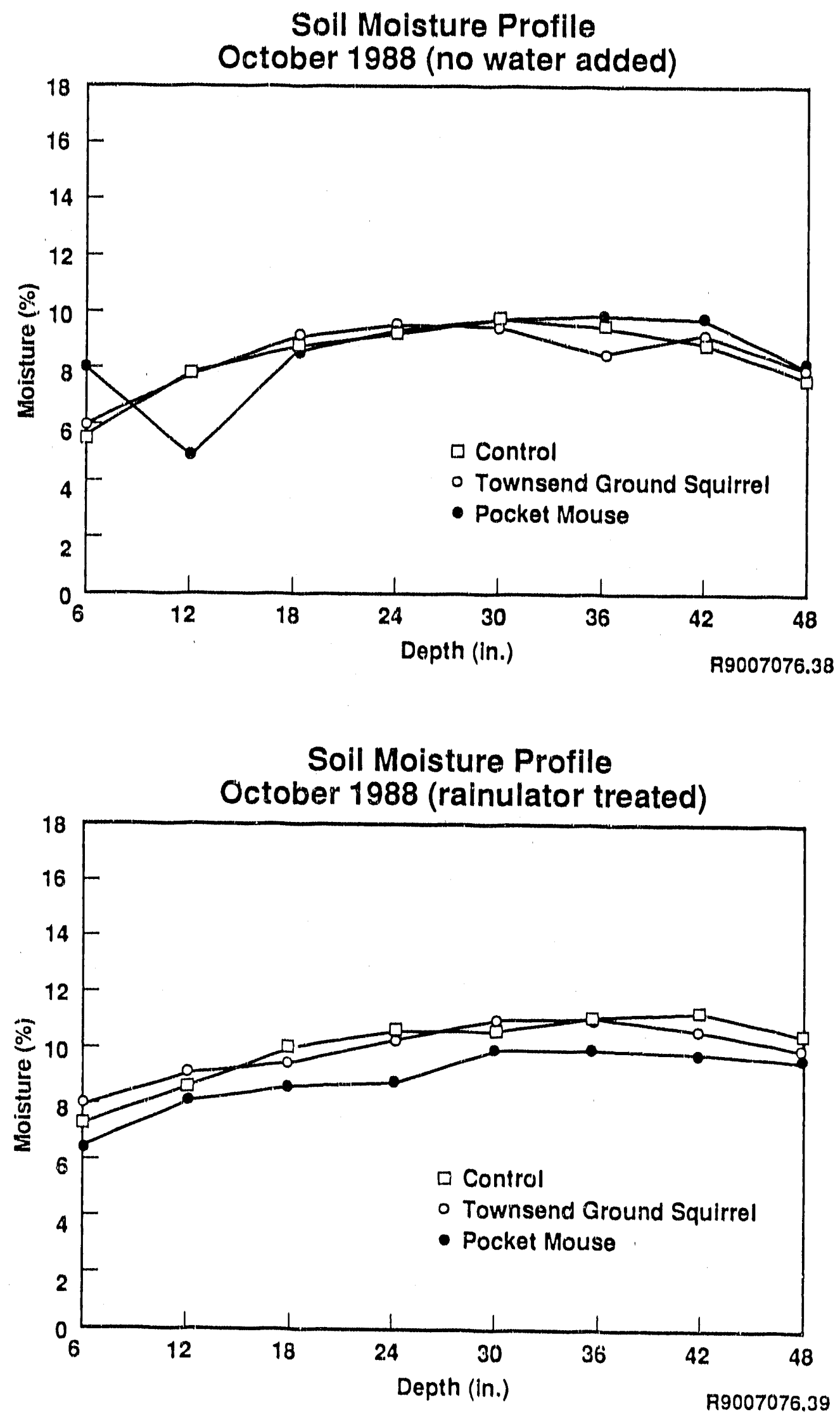
WHC -E.P-0299

APPENDIX C

TEST 2 HYDROPROBE DATA

$$
c-1 / C_{i i}
$$


WHC-EP-0299

TABLE SUMMARY OF HYOROPROAE REAOINOS OURING TEST WL

\begin{tabular}{|c|c|c|c|c|c|c|c|c|c|c|}
\hline & & NOV18 & DeCO & JANS & JAN31 & MAR 10 & MAR2O & MAR28 & APA 7 & APA12 \\
\hline BOX $1 A P O$ & DEPTH & & & & & & & & & \\
\hline \multirow[t]{6}{*}{ PROBE 11} & 5 & 3591 & 3243 & 3199 & 2158 & 3531 & 2969 & 2943 & 2003 & 2467 \\
\hline & 11 & 4782 & 4091 & 4896 & 4283 & 8223 & 7929 & 6668 & 5394 & 5127 \\
\hline & 17 & 4589 & 4194 & 4467 & 4447 & 7602 & 7428 & 7110 & 3750 & 5358 \\
\hline & 23 & 4556 & 4537 & 4942 & 4519 & 6101 & 7363 & 7070 & 6406 & 6181 \\
\hline & 29 & 4622 & 4371 & 4647 & 4465 & 4508 & 5940 & 6193 & 6087 & 6338 \\
\hline & 35 & 4704 & 4421 & 4719 & 4594 & 4689 & 4878 & 5207 & 5365 & 5625 \\
\hline
\end{tabular}

BOX IA PO

PROBE 12

DEPTH

53750

3750

$11 \quad 4550$

$17 \quad 4486$

450

4365

$3730 \quad 1764$

5172

2857

3746

$2740 \quad 2780$

4314

$4766 \quad 4326$

9032

80194

7010

$5942 \quad 5490$

23

4208

$4281 \quad 4348$

8826

6379

7542

6896

6462

29

4254

$4456 \quad 4331$

7379

7660

7905

7001

6601

35

$4229 \quad 4412$

$4285 \quad 4179$

5672

6236

6213

$6246 \quad 6234$

BOK IA PO

DEPTH

AVERAGE

5
11
17
23
29
35

$3671 \quad 2714$

$4666 \quad 4220$

$3463 \quad 1961$

$4831 \quad 4305$

4305

4379

4398

4468

$4538 \quad 4254$

$4699 \quad 4425$

4392

4352

8628

2913

8250

7904

6740

7516

$4336 \quad 4263$

4487

5135

6028

4996

$5200 \quad 5403$

$80 \times 10 \mathrm{CO}$

DEPTH

PROBE 1,3

3364

4387

4579

4805

3345

2372

2024

$6839 \quad 5608 \quad 5309$

7326

$6323 \quad 5910$

$6704 \quad 6391$

7288

$6172 \quad 6286$

$4467 \quad 4417$

6203

$5287 \quad 5514$

$\begin{array}{llllllllll}5 & 3922 & 2610 & 3364 & 3007 & 4793 & 2793 & 3864 & 2647 & 2575 \\ 11 & 4759 & 4673 & 5123 & 4659 & 9772 & 8513 & 7517 & 6333 & 5806 \\ 17 & 4695 & 4645 & 4964 & 4674 & 9689 & 8853 & 8144 & 7105 & 6806 \\ 23 & 4679 & 4795 & 4996 & 4739 & 9890 & 9051 & 8583 & 7831 & 7476 \\ 29 & 4762 & 4747 & 5035 & 4822 & 9961 & 8710 & 8198 & 7546 & 7340 \\ 35 & 4934 & 4890 & 5169 & 4873 & 6060 & 7146 & 7297 & 7150 & 6899\end{array}$

$80 \times 10 \mathrm{CO}$

DEPTH

PROBE 14

5
11
17
23
29
35

$\begin{array}{ll}3319 & 1868 \\ 4196 & 3899 \\ 4660 & 4435 \\ 4620 & 4701 \\ 4386 & 4379 \\ 4626 & 4803\end{array}$

$\begin{array}{ll}2991 & 1754 \\ 4382 & 4148 \\ 4792 & 4043 \\ 4962 & 4501 \\ 4374 & 4280 \\ 4830 & 4870\end{array}$

5850
6586
5133
4736
4388
4973

4302
6713
5663
4766
4384
4793

388
6135
595
5291
4.568
470

3232

2632

5020

5933

$5291 \quad 5681 \quad 5585$

$4.568 \quad 4583 \quad 4838$

$4781 \quad 4776 \quad 4925$ 
WHC-EP-0299

HOX 10 CO UEPTH AVERAOB

$\begin{array}{llllllllll}5 & 3621 & 2239 & 3178 & 2381 & 9322 & 3548 & 3476 & 2940 & 2604 \\ 11 & 4477 & 4286 & 4753 & 4404 & 8479 & 7613 & 6826 & 5805 & 5413 \\ 17 & 4670 & 4540 & 4870 & 4659 & 7411 & 7258 & 7051 & 6544 & 6370 \\ 23 & 4690 & 4748 & 4929 & 4620 & 7313 & 6909 & 6937 & 6756 & 6536 \\ 29 & 4574 & 4563 & 4705 & 4551 & 7175 & 6547 & 6383 & 6070 & 6089 \\ 35 & 4780 & 4847 & 5004 & 4872 & 5517 & 5970 & 6039 & 5963 & 5912\end{array}$

BOX 1C PM

DEPTH

PROBE 15

5
11
17
23
29
35

2858

3913

3348

2.641

5987

3874
8141

$\begin{array}{lll}4718 & 3321 & 1678\end{array}$

$4271 \quad 4298$

4510

4492

$4652 \quad 4362$

4,604

4383

0339

8239

7477

6077

5200

4442

4562

4604

5369

7059

7468

$6616 \quad 6258$

4772

4920

4087

4503

4696

6142

7060

$6561 \quad 6444$

1,680

5009

5091

6563

$6220 \quad 6196$

$5595 \quad 5883 \quad 5716$

BOX 1C PM DEPTH

PROUE 16

$\begin{array}{llllllllll}5 & 3019 & 3163 & 3194 & 2674 & 740 & 3601 & 3288 & 2593 & 2862 \\ 11 & 4257 & 4204 & 4190 & 4041 & 8184 & 7442 & 6622 & 5920 & 5690 \\ 17 & 4685 & 4648 & 4610 & 4448 & 7895 & 8130 & 7558 & 7257 & 7036 \\ 23 & 4657 & 4597 & 4702 & 4698 & 5440 & 7030 & 7113 & 6775 & 6600 \\ 29 & 4569 & 4555 & 4722 & 4557 & 4586 & 5352 & 5711 & 5939 & 6080 \\ 35 & 4753 & 4808 & 4589 & 4642 & 4739 & 4567 & 4836 & 5188 & 5313\end{array}$

BOX 1C PM

DEPTH

AVERAUE

5
11
17
23
35

$2939 \quad 3338 \quad 3271$

$4341 \quad 4371 \quad 4430$

$4478 \quad 4473 \quad 4560$

$4655 \quad 10480 \quad 4553$

4506

4559

4663

4763

4064

4788

2.658

4311

4470

4541

4530

4661

$6364 \quad 3738$

3738

$8666 \quad 7792 \quad 7050$

7050

$8117 \quad 8185$

$5408 \quad 7045$

4641

7045

7087

6137

NOV18

DEC

JAN

JAN3

MAR

874

4829

5216

$\begin{array}{ll}2957 & 2270 \\ 5999 & 5449 \\ 6937 & 6547 \\ 6668 & 6522 \\ 6080 & 6138 \\ 5436 & 5515 \\ & \\ \text { APR7 } & \text { APR12 }\end{array}$

HOX $2 \mathrm{APO}$

DEPTH

PkOBE 21

5
11
17
23
29
35

$\begin{array}{lll}2363 & 3208 & 4397 \\ 4178 & 4098 & 4193 \\ 4351 & 4454 & 4394 \\ 4690 & 4575 & 4375 \\ 4614 & 4491 & 4101 \\ 4337 & 4412 & 4234\end{array}$

2141
4150
4326
4561
4401
4496

4798
8238
7526
5271
4778
4730

3349
7379
7562
6874
5462
4690

3397
658
712
675
5976
4990

$\begin{array}{ll}3176 & 1742 \\ 5744 & 4845 \\ 6458 & 6316 \\ 6547 & 6643 \\ 6114 & 6187 \\ 5362 & 5413\end{array}$


WHC-EP-0299

\begin{tabular}{|c|c|c|c|c|c|c|c|c|c|c|}
\hline$B O X \quad 2 A P A$ & DEPTH & & & & & & & & & \\
\hline \multirow[t]{6}{*}{ PROQE 22} & $\mathfrak{5}$ & 2992 & 1879 & 2769 & 252.4 & 8133 & 5963 & 5366 & 36.33 & 3763 \\
\hline & 11 & 3250 & 2803 & 2945 & 2975 & 8225 & 7315 & 6291 & 5435 & 4935 \\
\hline & 17 & 3213 & 2984 & 2896 & $302 . "$ & 6120 & 6219 & 5724 & 5354 & $\$ 190$ \\
\hline & 23 & 4076 & 3963 & 3973 & 1,000 & 4392 & 4773 & 9049 & 5437 & 5499 \\
\hline & 29 & 4631 & 4212 & 4465 & 4409 & 4520 & 4525 & 5047 & 5298 & 5454 \\
\hline & 35 & 4386 & 3961 & 4094 & 4112 & 4231 & 4425 & 4382 & 4529 & 460166 \\
\hline$B O X \quad 2 A P O$ & DEPTH & & & & & & & & & \\
\hline \multirow[t]{6}{*}{ AVERAQR } & 5 & 2658 & 254.4 & 356.3 & 2333 & 6466 & 4656 & 4382 & 3430 & 2753 \\
\hline & 11 & 3714 & 3431 & 3564 & 3563 & 8232 & 7347 & 6439 & 5590 & 4890 \\
\hline & 17 & 3782 & 3719 & 3645 & 3674 & 6023 & 68889 & 61,23 & 5906 & 5753 \\
\hline & 23 & 4383 & 4269 & 4174 & 4281 & 4832 & 5824 & 5904 & 5992 & 6071 \\
\hline & 29 & 4623 & 4352 & 4283 & 1,405 & 4649 & 49074 & 5512 & 5706 & 5821 \\
\hline & 35 & 4362 & 4187 & 4164 & 4304 & 4481 & 4558 & 4686 & 4946 & 5050 \\
\hline
\end{tabular}

$\begin{array}{lllllllllll}\text { BOX 28 CO } & \text { DEPTH } & & & & & & & \\ \text { PROBE: 23 } & 5 & 4085 & 4195 & 4360 & 4040 & 5440 & 4452 & 5612 & 4096 & 3696 \\ & 11 & 4862 & 5065 & 5595 & 5388 & 9590 & 0484 & 7733 & 6025 & 6526 \\ & 17 & 5220 & 5133 & 5549 & 5291 & 8767 & 8442 & 0002 & 7366 & 7002 \\ & 23 & 5109 & 5028 & 5169 & 50146 & 6288 & 7744 & 7408 & 7126 & 6858 \\ & 29 & 4747 & 4999 & 5002 & 4947 & 5322 & 6813 & 7038 & 6912 & 6793 \\ & 35 & 5140 & 5275 & 5171 & 5047 & 5199 & 5976 & 6697 & 6708 & 6820\end{array}$

BOX 28 CO DEPTH

PROBE 24

$\begin{array}{llllllllll}5 & 3691 & 3652 & 3301 & 2679 & 7177 & 3520 & 5206 & 3329 & 3218 \\ 11 & 4007 & 4148 & 4479 & 4349 & 10351 & 8843 & 7633 & 6811 & 6556 \\ 17 & 4847 & 4750 & 4831 & 4704 & 11419 & 9418 & 8466 & 7950 & 7596 \\ 23 & 5014 & 5140 & 5430 & 5010 & 8301 & 8502 & 8054 & 7707 & 7620 \\ 29 & 4612 & 4694 & 4669 & 4752 & 6336 & 5600 & 6739 & 6673 & 6615 \\ 35 & 4932 & 4897 & 4696 & 4748 & 5394 & 5101 & 5369 & 5913 & 5886\end{array}$

BOX $2 B C O$

AVERAGE
DEPTH

11

17

23.

29

35

$\begin{array}{ll}3888 & 3924 \\ 4435 & 4607 \\ 5034 & 4942 \\ 5062 & 5084 \\ 4680 & 4847 \\ 5036 & 5086\end{array}$

3831
5037
5190
5300
4836
4909

$\begin{array}{rr}3360 & 6309 \\ 4869 & 9971 \\ 4998 & 10093 \\ 5028 & 729 \\ 4850 & 5829 \\ 4898 & 5297\end{array}$

$\begin{array}{llll}3986 & 5409 & 3713 & 3457 \\ 8664 & 7603 & 6818 & 6541 \\ 8930 & 8234 & 7658 & 7299 \\ 8123 & 7731 & 7417 & 7239 \\ 5707 & 6809 & 6793 & 6704 \\ 5539 & 6033 & 6311 & 6353\end{array}$


WHC-E.P.0299

BOX 26: $\mathrm{PO}$ DEPTH

PHOBE 2.5

$\begin{array}{llllllllll}5 & 2.695 & 2646 & 3611 & 3091 & 5727 & 4472 & 3558 & 3414 & 2183 \\ 11 & 3977 & 4099 & 4130 & 4051 & 7232 & 7205 & 6687 & 5995 & 5603 \\ 17 & 4172 & 4123 & 4272 & 3988 & 5428 & 7076 & 6976 & 6719 & 6712 \\ 23 & 4598 & 4361 & 4542 & 4385 & 4407 & 5680 & 6302 & 6375 & 6335 \\ 29 & 4435 & 4366 & 4657 & 4418 & 4550 & 4657 & 5252 & 5577 & 5785 \\ 35 & 4598 & 4705 & 4813 & 4779 & 4824 & 4835 & 4914 & 5284 & 5407\end{array}$

BOX 2C PQ PROB: 26
DEPTH

11

17

23

29

35

BOX 2C PO AVERAOE
DEPTH

11

17

23

29

35
$2660 \quad 2593$

$3674 \quad 3696$

$4128 \quad 4054$

$4401 \quad 4340$

$4025 \quad 4068$

4204

4305

\section{$2636 \quad 2539$}

3293

3984

4319

3770

3904
2841

3384

3713

4167

3610

3988

3226

3757

3993

4355

4134

4401

$\begin{array}{ll}1315 & 5064 \\ 3037 & 7419 \\ 3708 & 5702 \\ 4218 & 4490 \\ 3667 & 3702 \\ 3993 & 4082\end{array}$

3134

6801

6969

5501

3792

4095

$2 ? .03$

3544

3848

4302

4043

4384
$5396 \quad 3803$

73267063

$5565 \quad 7023$

$4449 \quad 559$

$4126 \quad 4225$

4453

4465

$\begin{array}{lll}3884 & 2401 & 2594 \\ 6350 & 5504 & 5517 \\ 6897 & 6775 & 6581 \\ 6214 & 6610 & 6585 \\ 4384 & 4979 & 5354 \\ 4106 & 4217 & 4456\end{array}$

594

6501

6585

4456 
WHC-EP-0299

APPENDIX D

TEST 2 SOIL MOISTURE GRAPHS

D. 1 Dii 
WHC-EP-0299

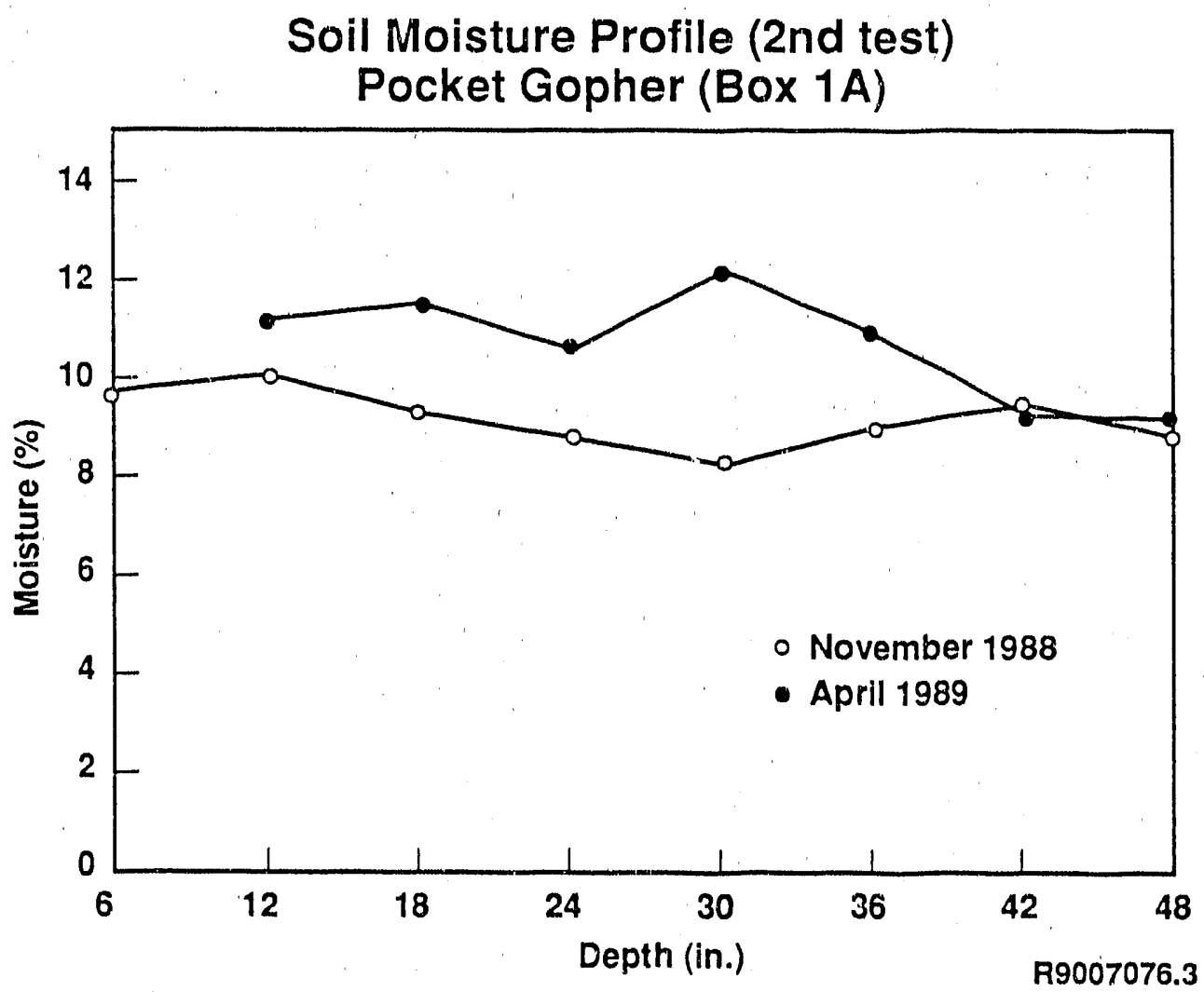

Soil Moisture Profile (2nd test)

Pocket Gopher (Box 2C)

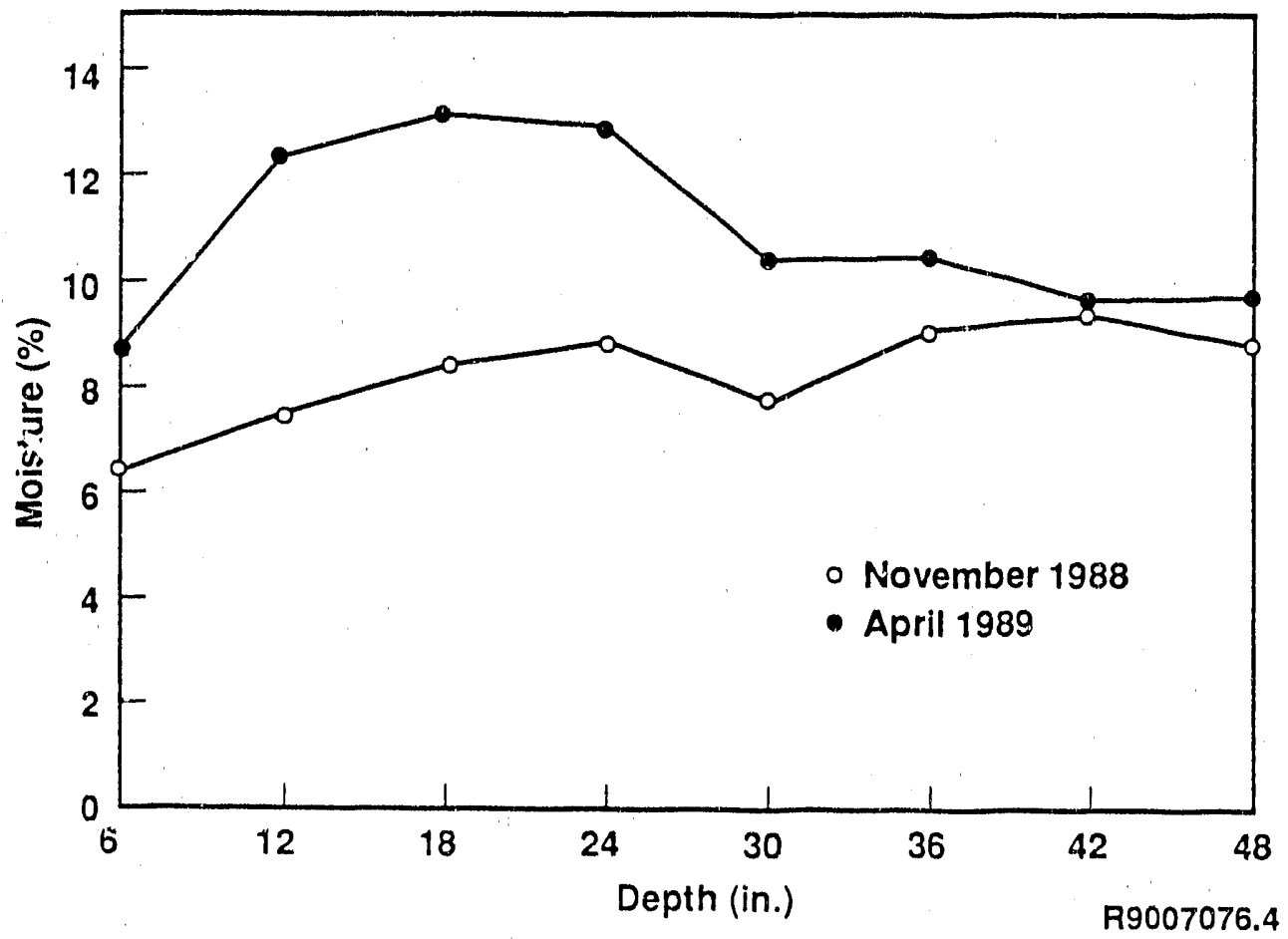


WHC-EP-0299

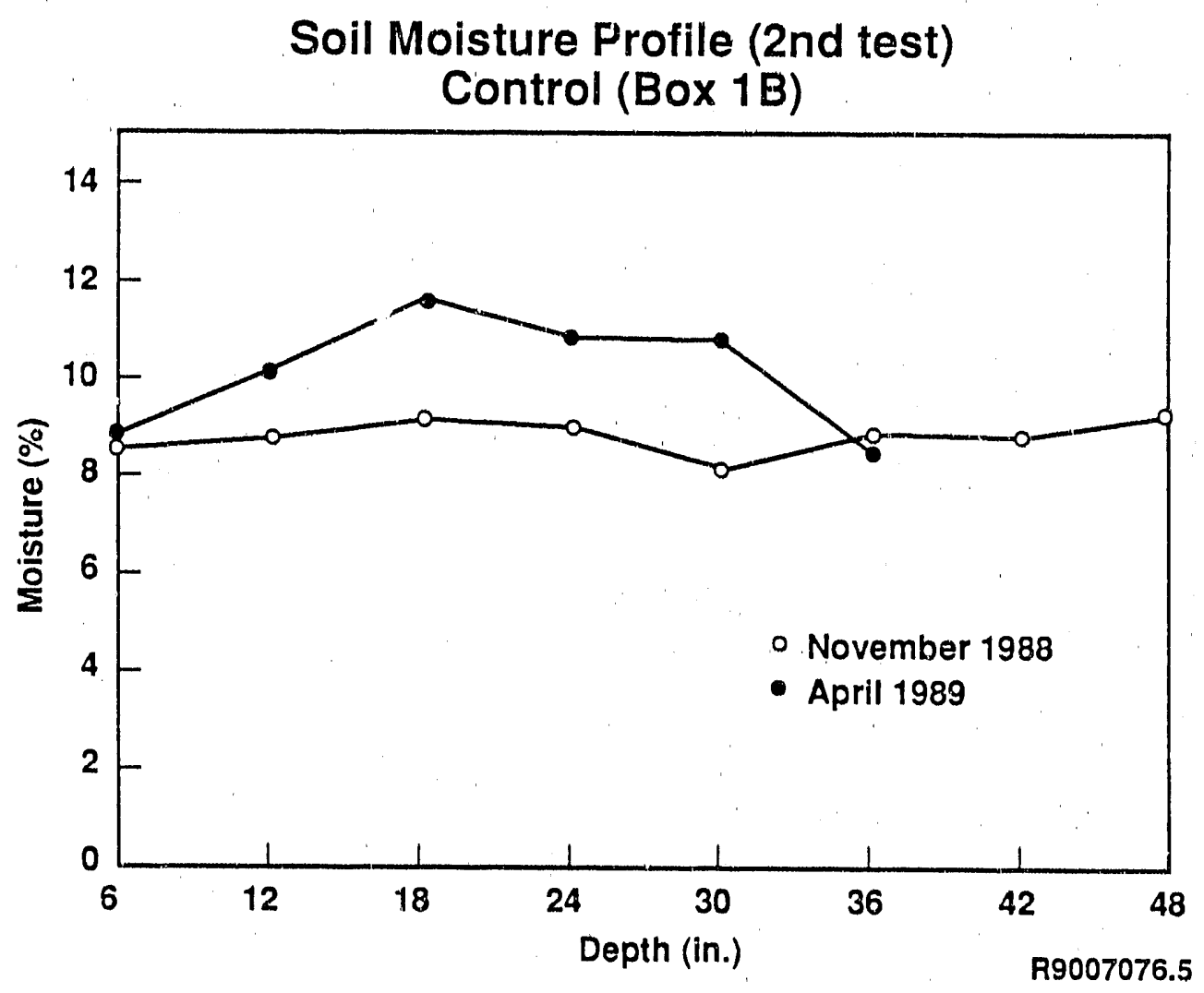

Soil Moisture Profile (2nd test)

Control (Box 2B)

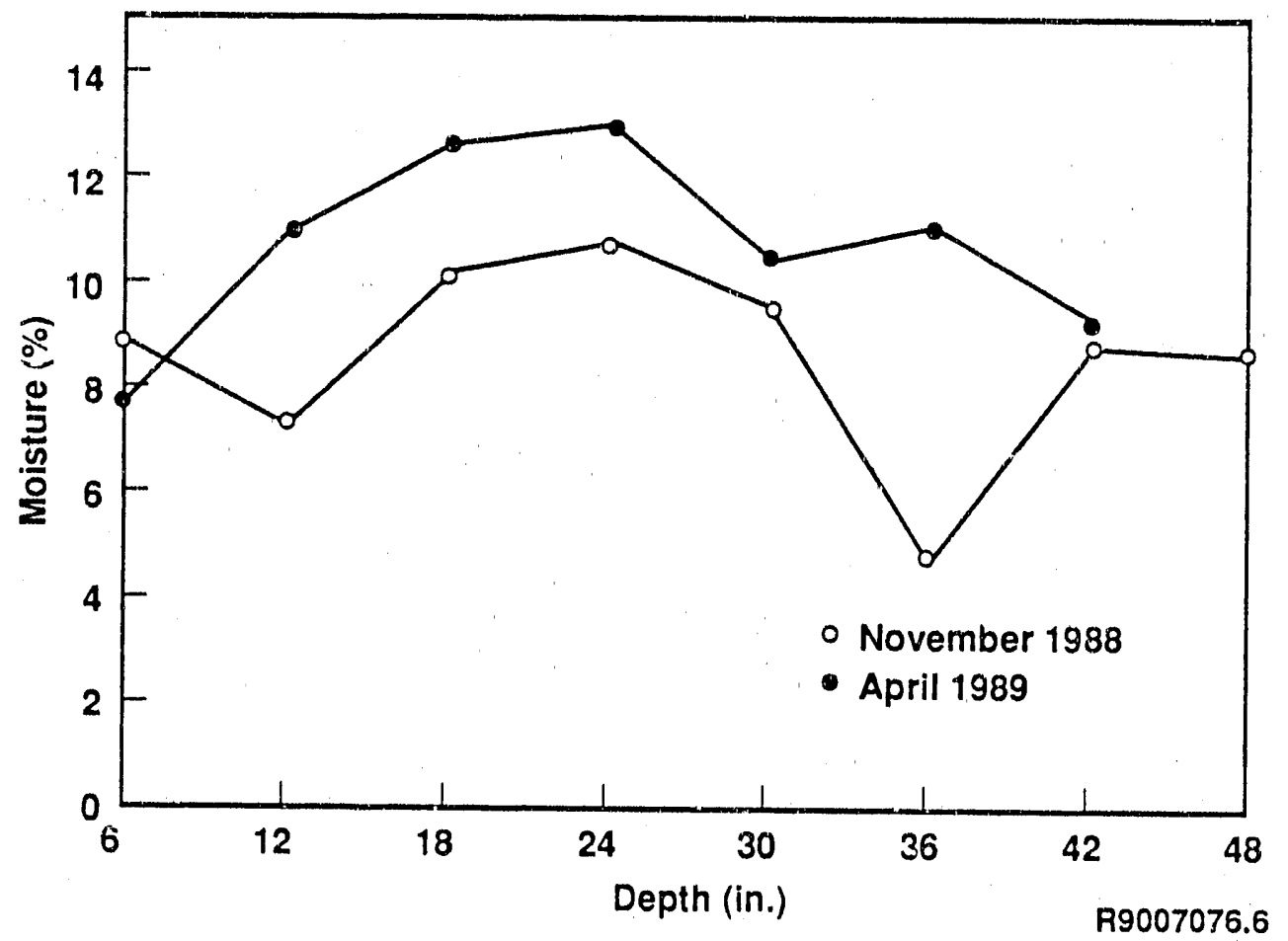


Soil Moisture Profile (2nd test)

November 1988 to April 1989 (0-6 in.)

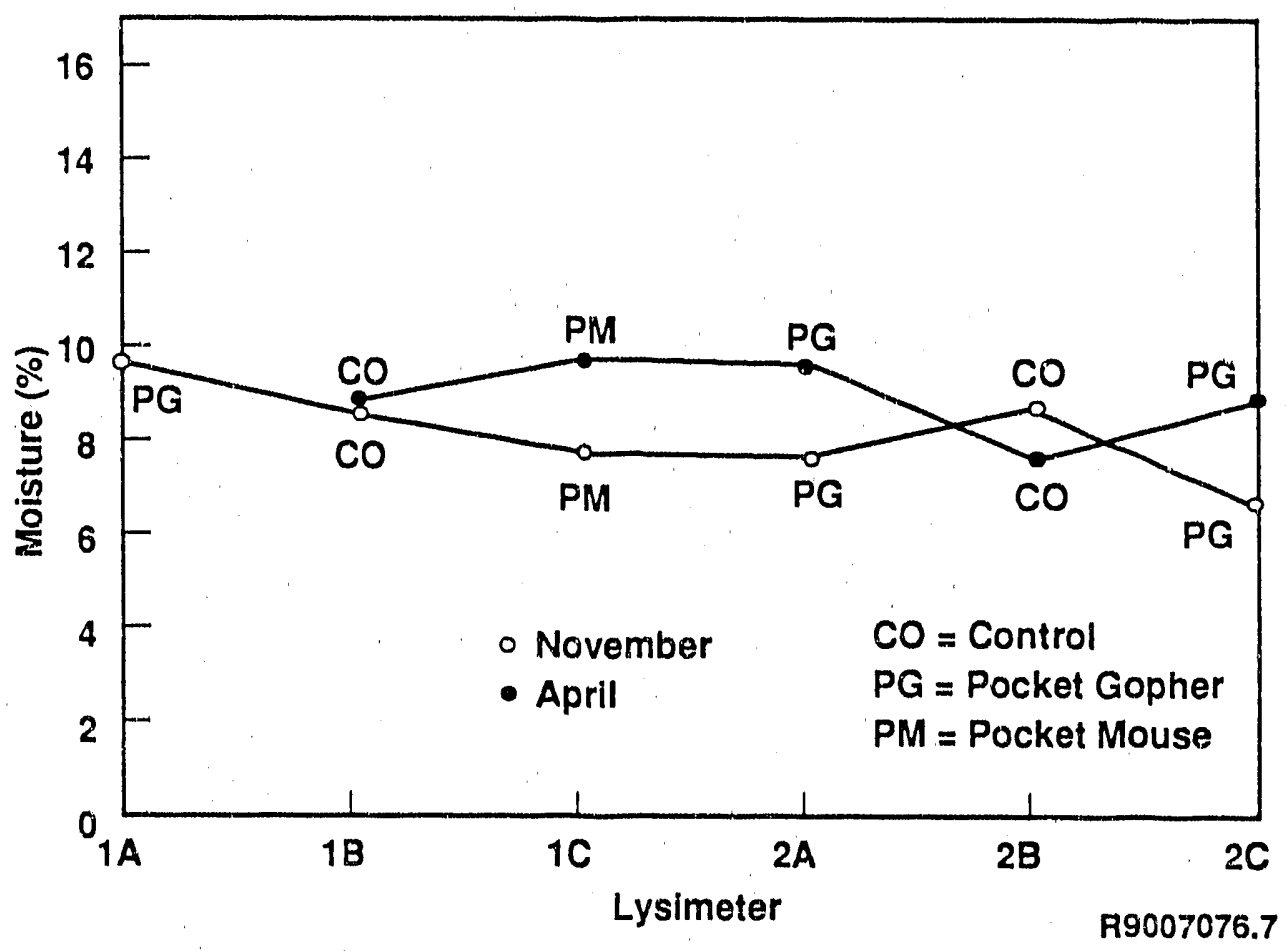

Soil Moisiure Profile (2nd test)

November 1988 to April 1989 (6-12 in.)

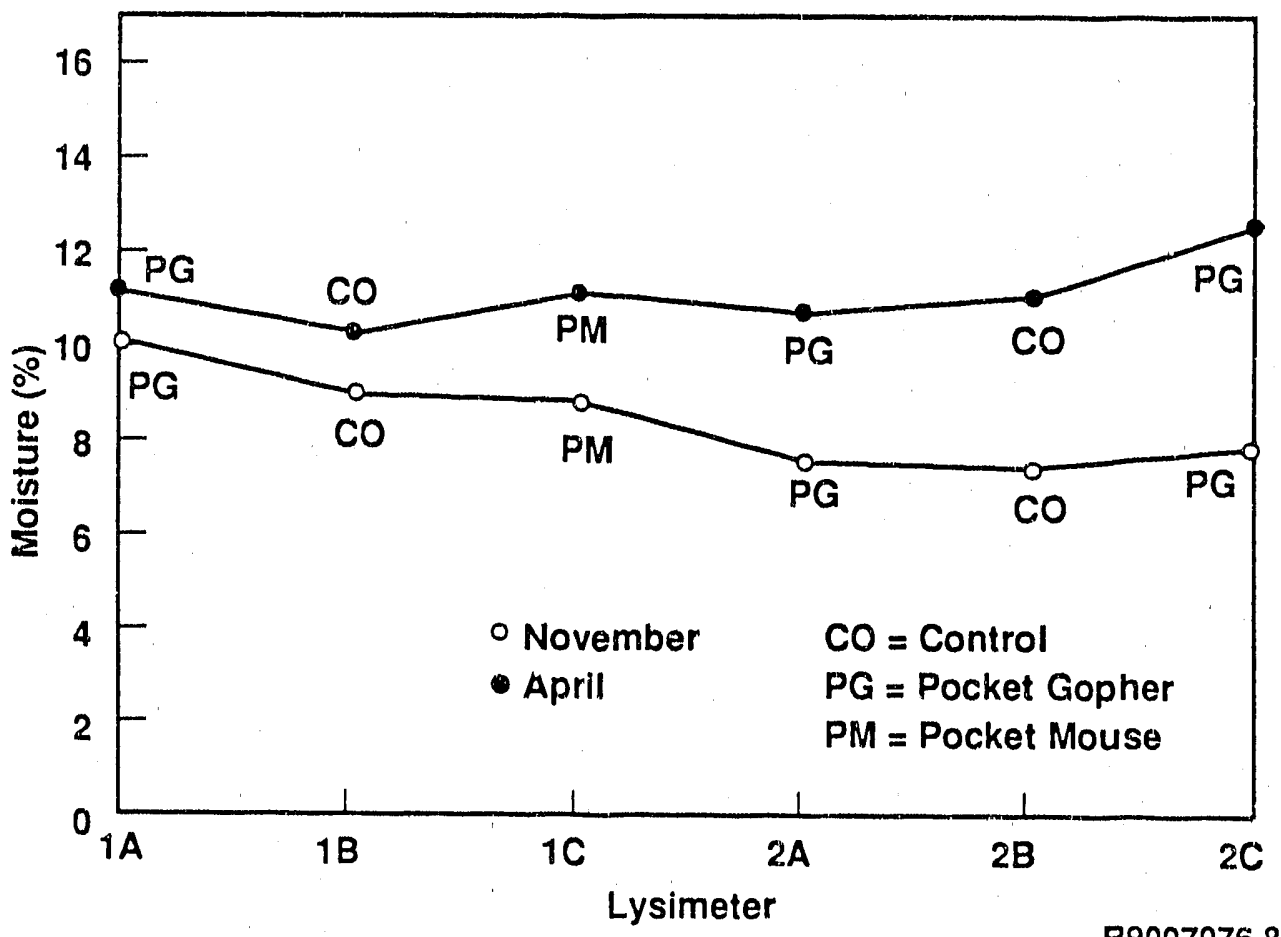


Soil Moisture Profile (2nd test)

November 1988 to April 1989 (12-18 in.)

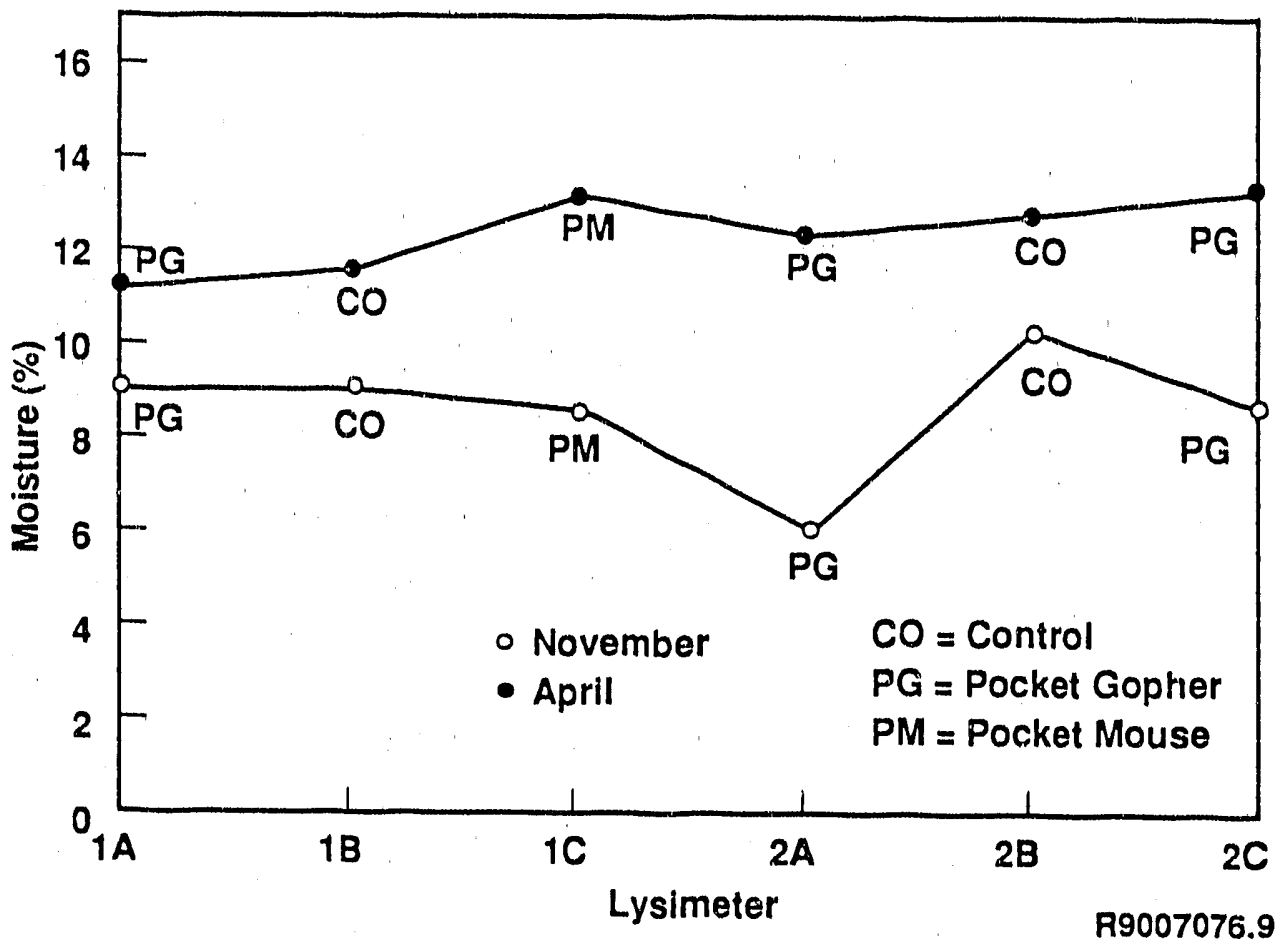

Soil Moisture Profile (2nd test) November 1988 to April 1989 (18-24 in.)

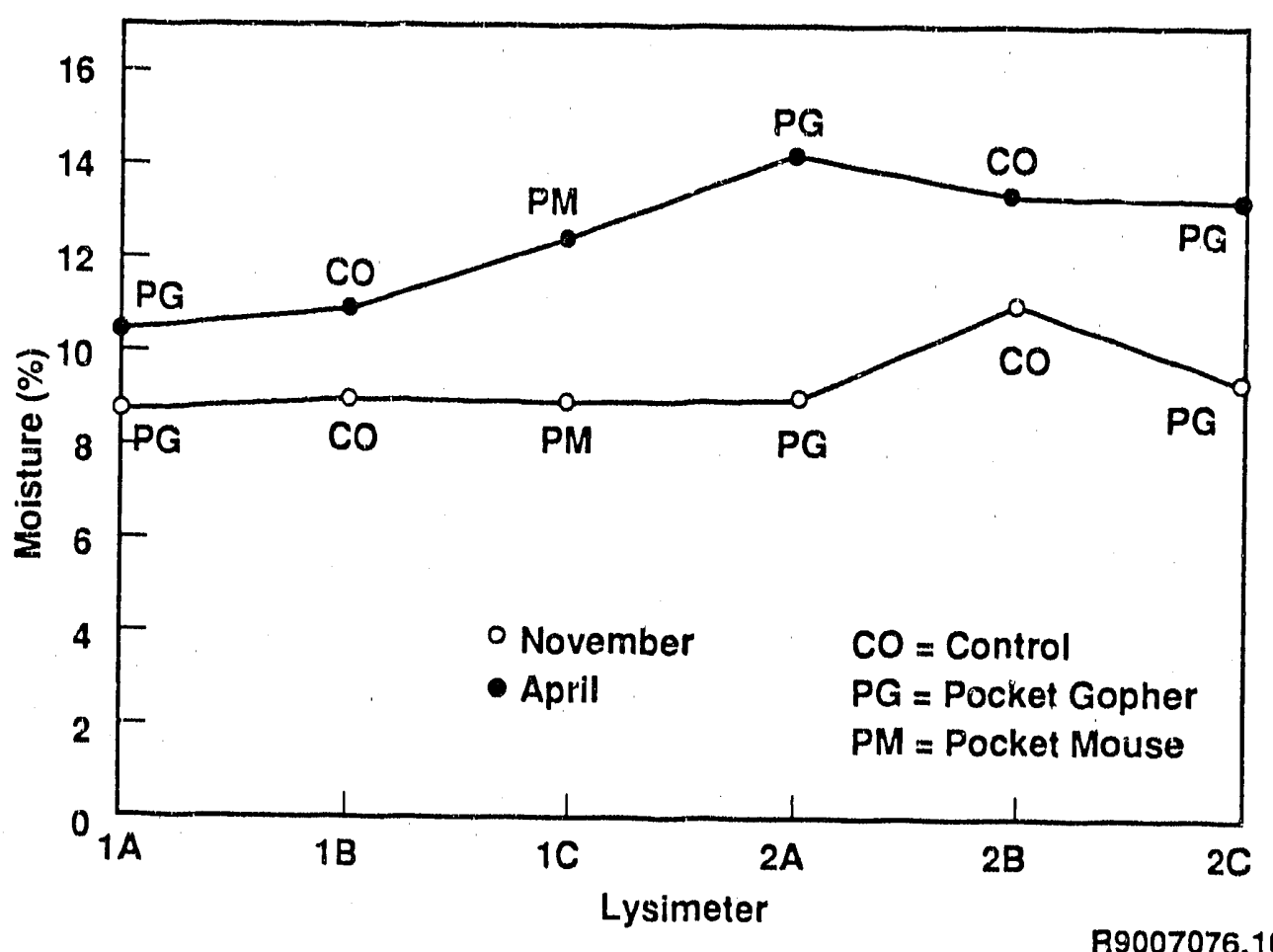

R9007076.10 
Soil Moisture Profile (2nd test)

November 1988 to April 1989 (24-30 in.)

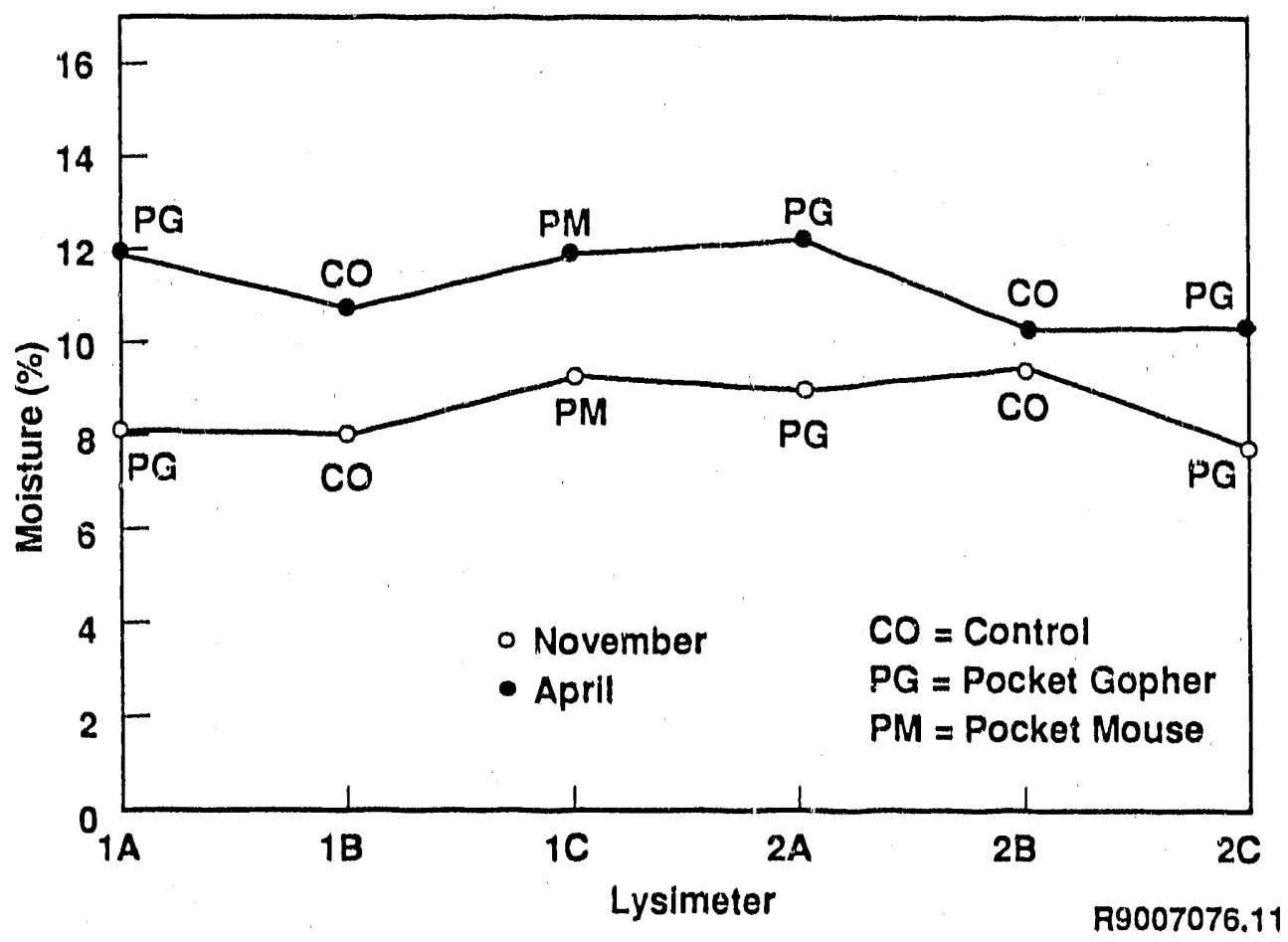

Soil Moisture Profile (2nd test)

November 1988 to April 1989 (30-36 in.)

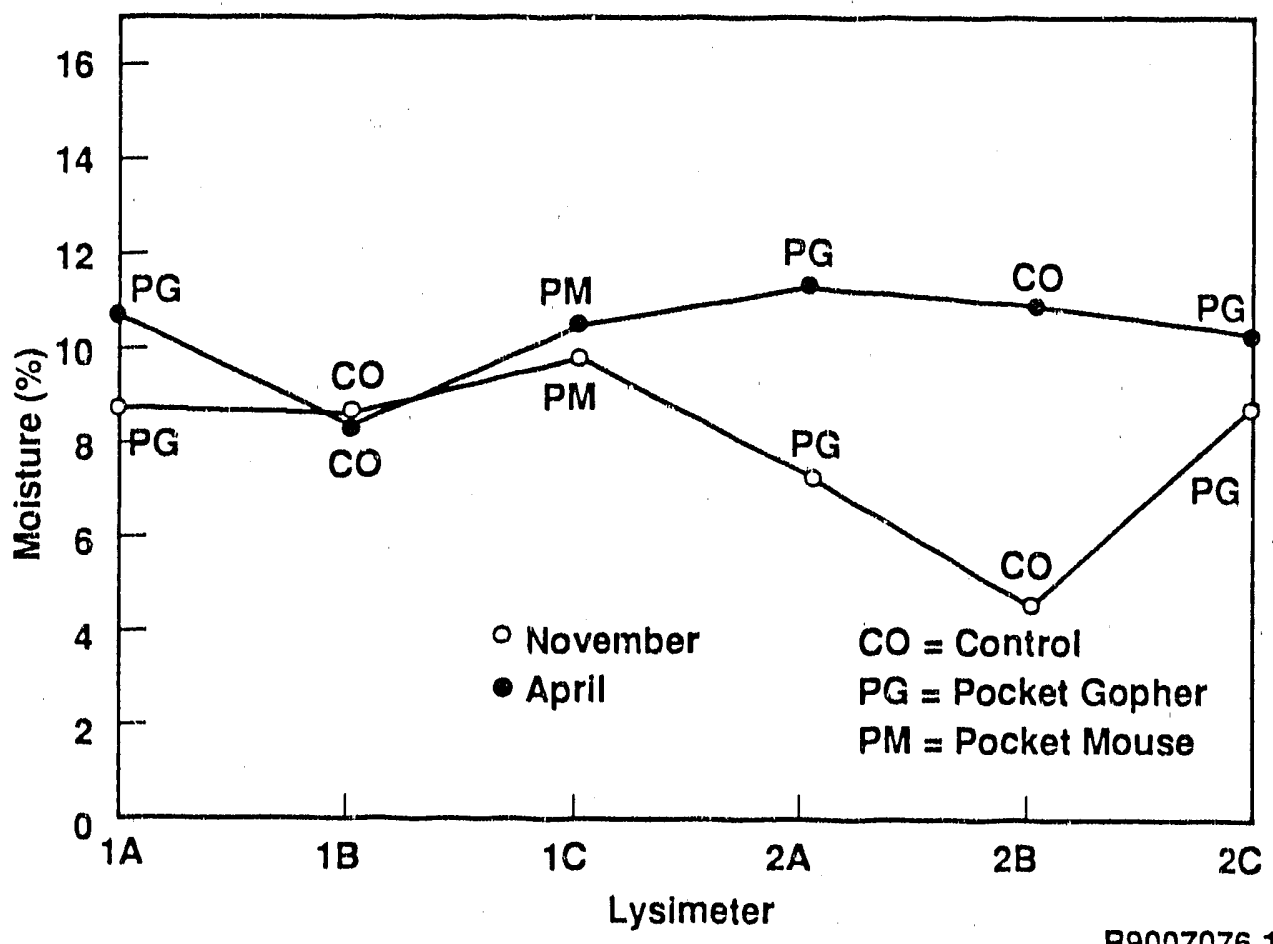

R9007076.12 
Soil Moisture Profile (2nd test)

November 1988 to April 1989 (36-42 in.)

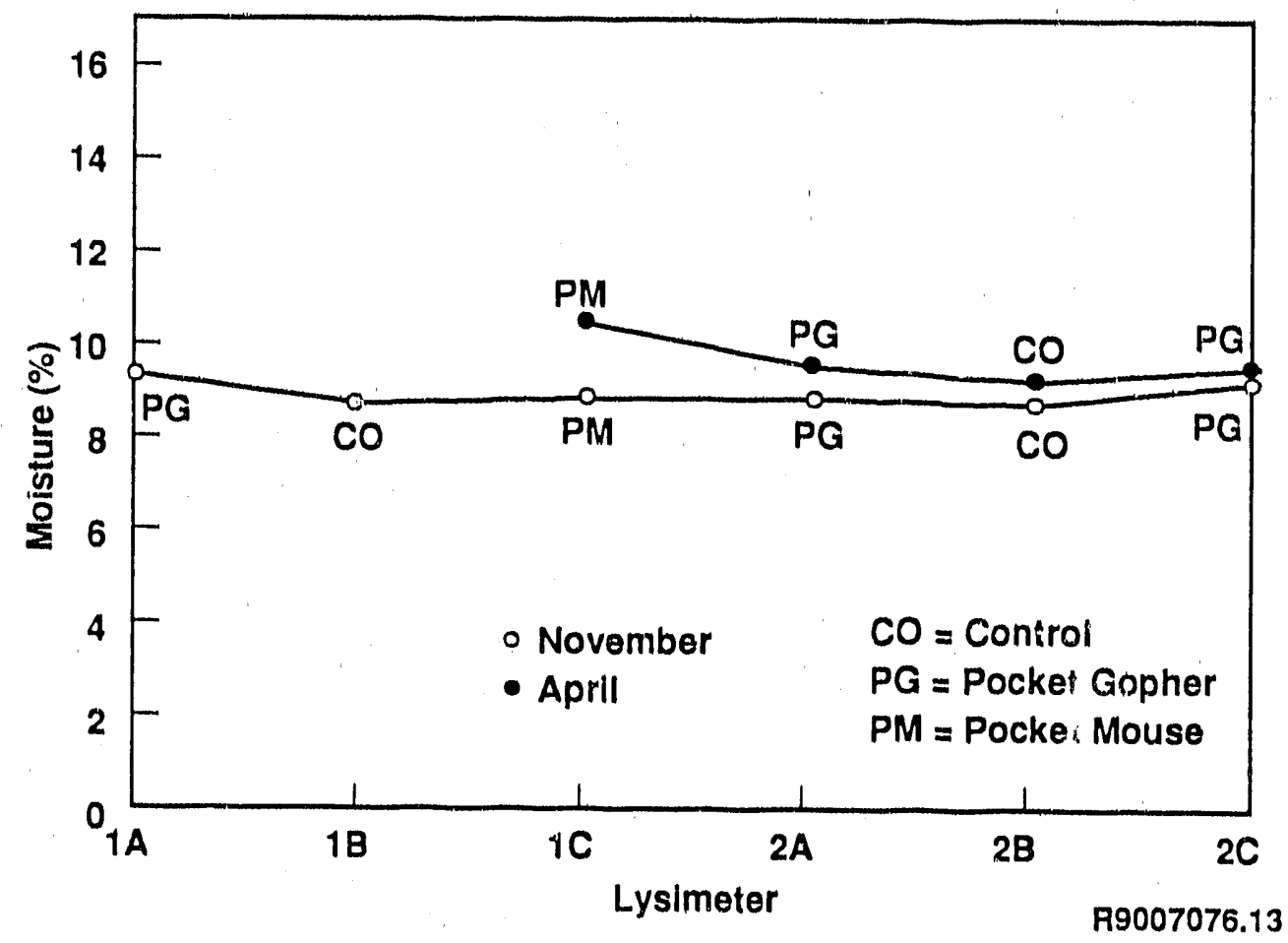

Soil Moisture Profile (2nd test)

November 1988 to April 1989 (42-48 in.)

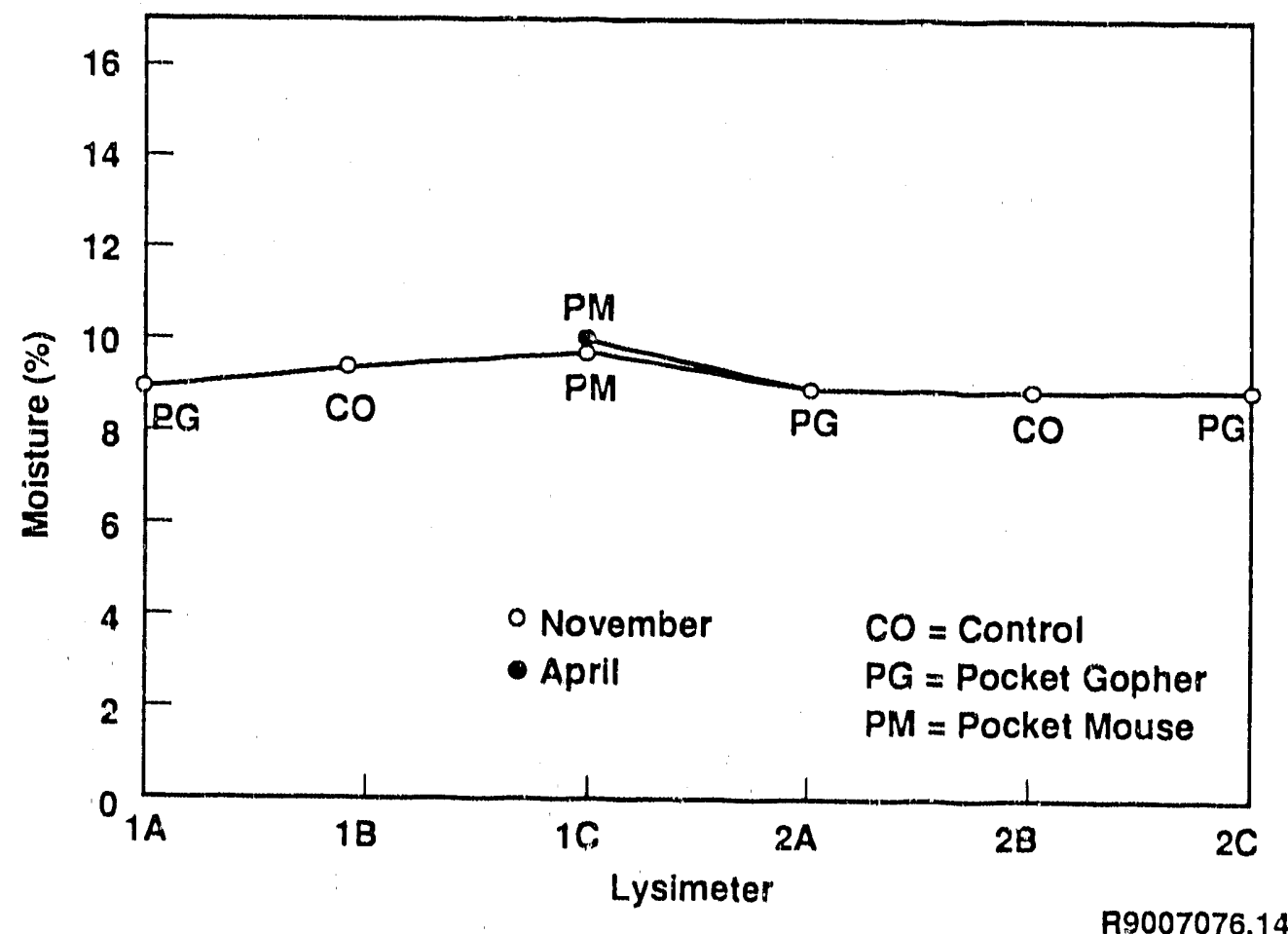


WHC-EP-0299

Soil Moisture Profile

April 1989 (end 2nd test)

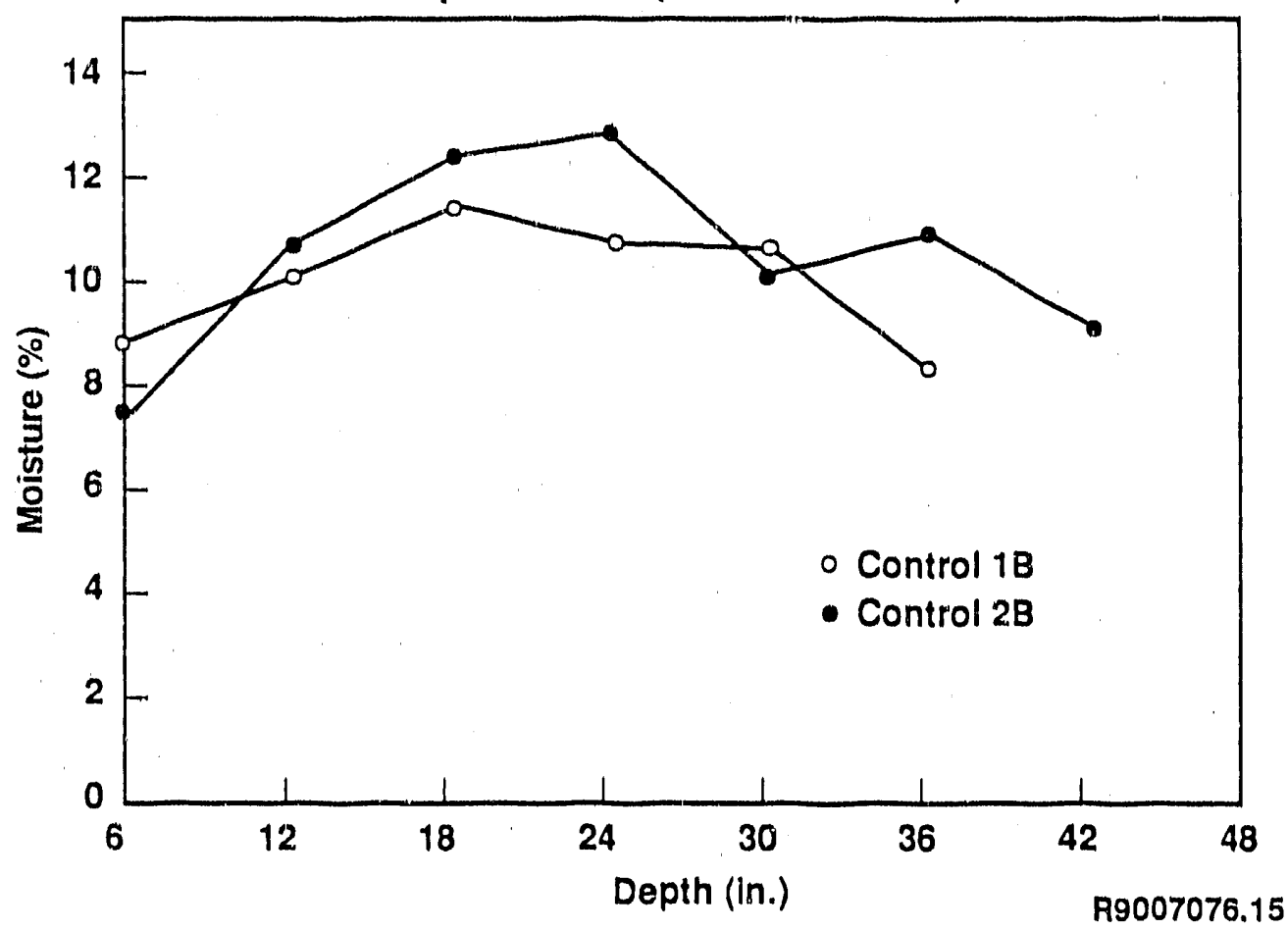

Soil Moisture Profile

April 1989 (end 2nd test)

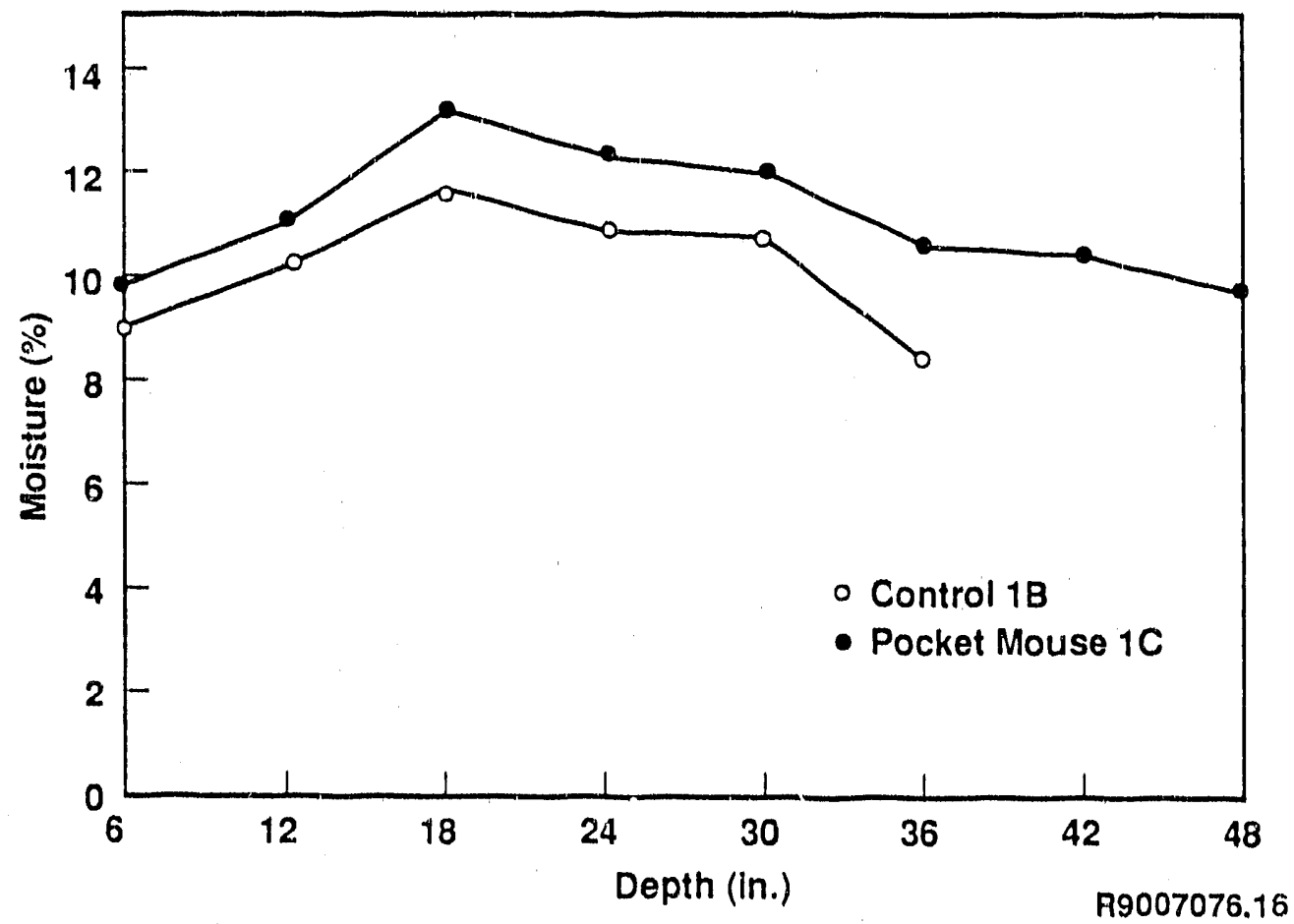


WHC-EP-0299

Soil Moisture Profile

April 1989 (end 2nd test)

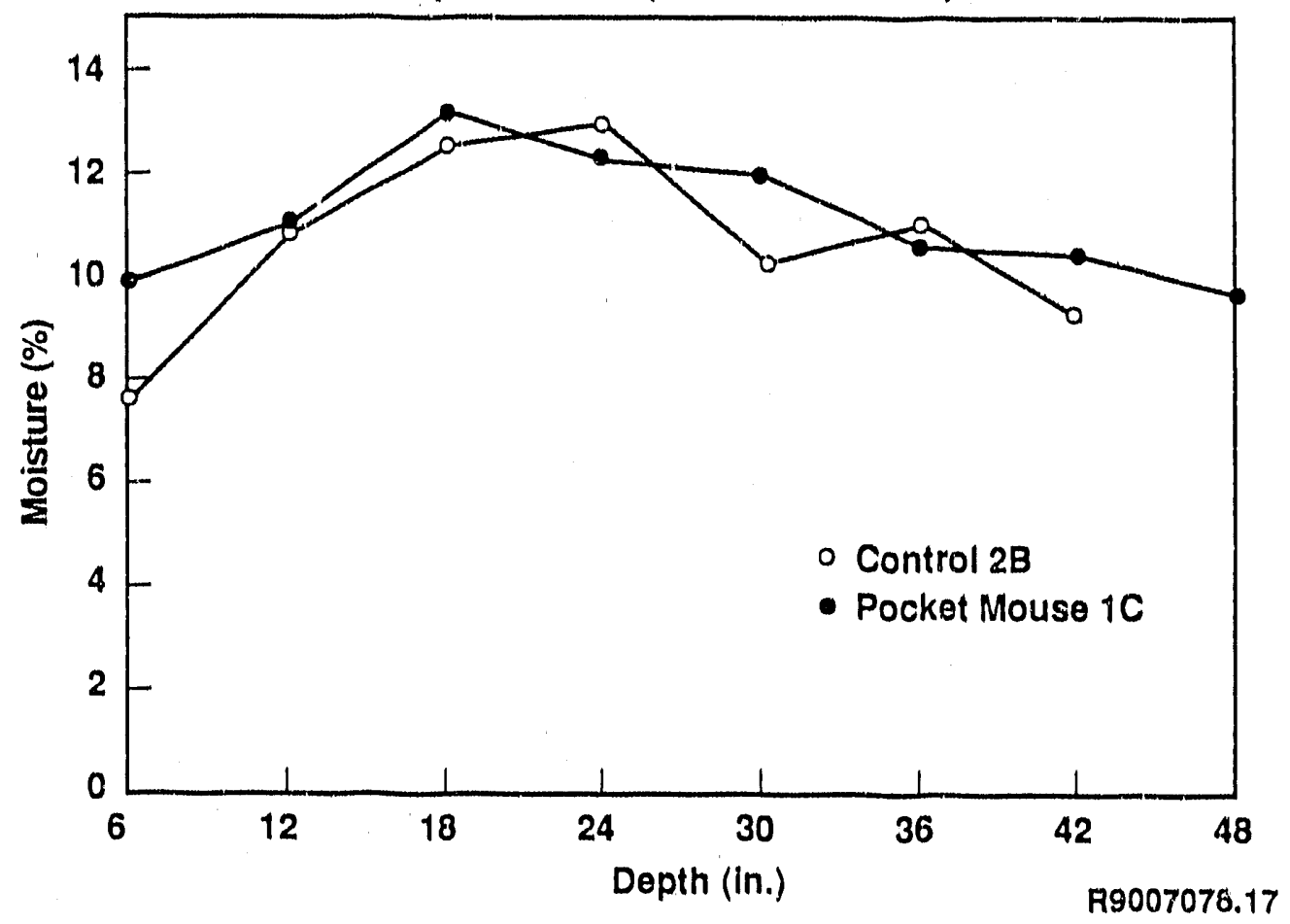

Soil Moisture Profile

April 1989 (end 2nd test)

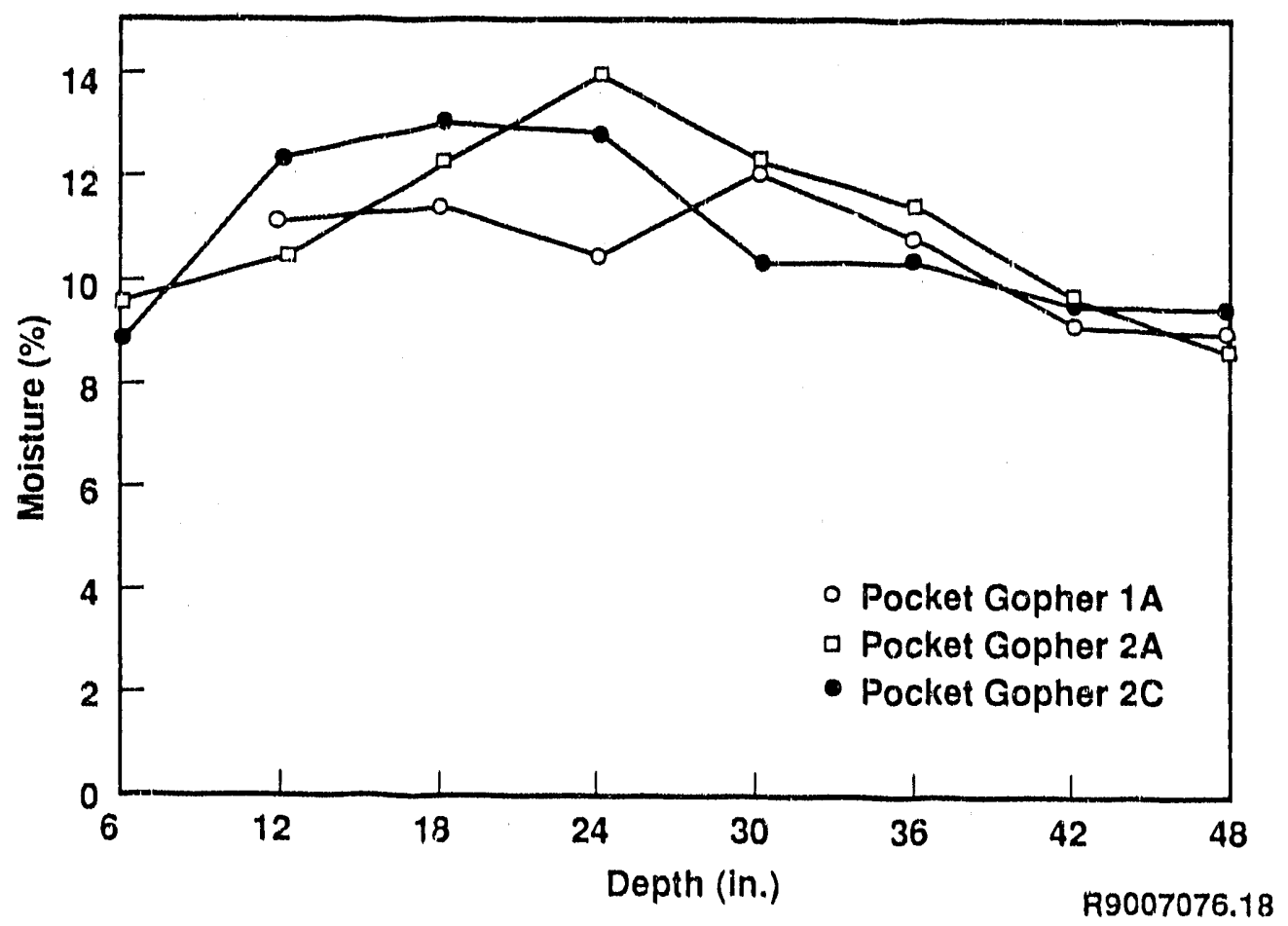


WHC-EP-0299

Soll Moisture Profile

April 1989 (end 2nd test)

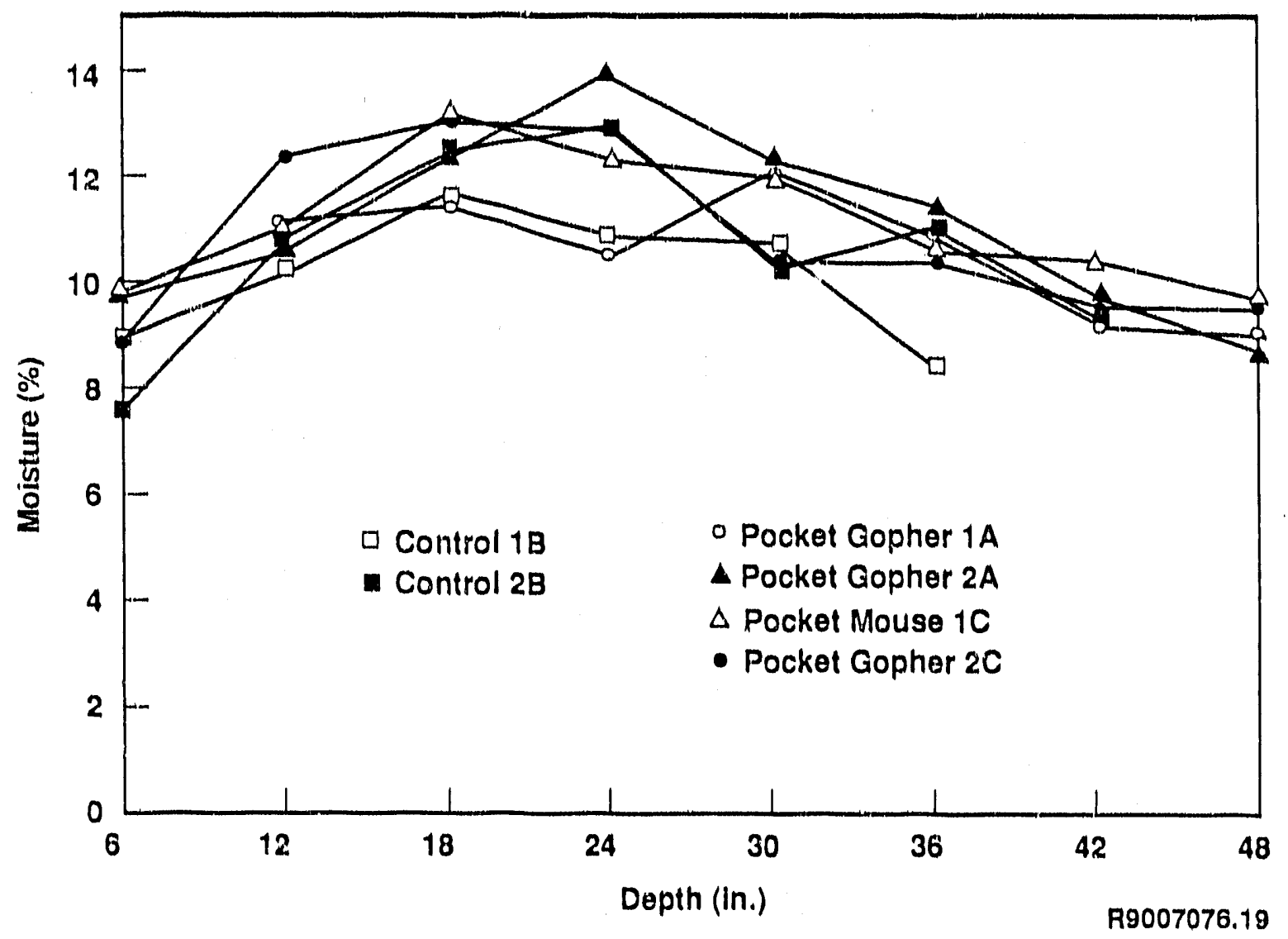

$0.9 / D-10$ 

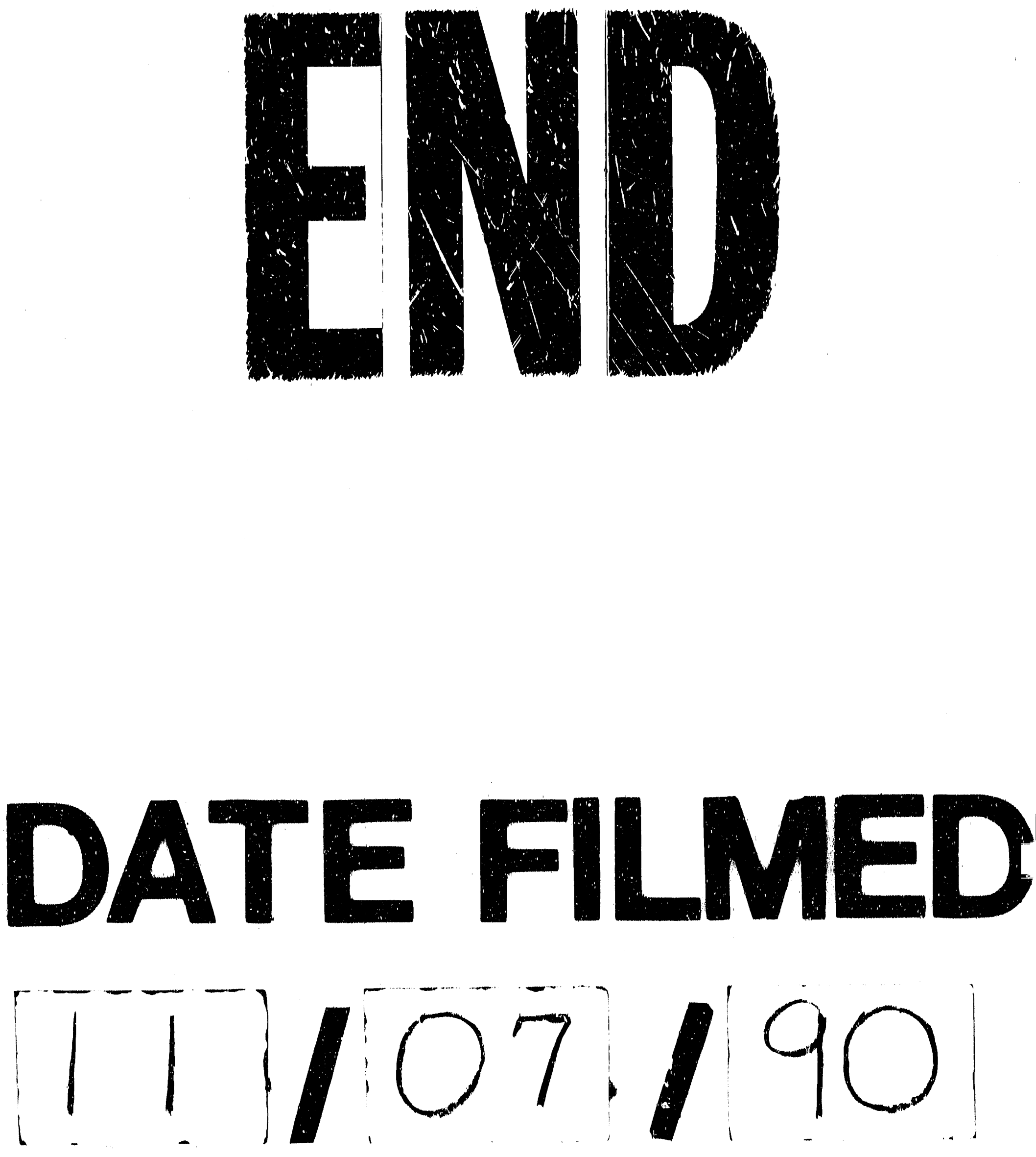
